\title{
SHOCKING STUFF
}

\section{TECHNOLOGY,} HOURS, AND FACTOR SUBSTITUTION

by Cristiano Cantore, Miguel A. León-Ledesma, Peter McAdam and Alpo Willman 
EUROSYSTEM

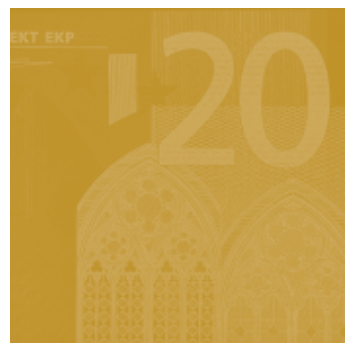

\section{WORKING PAPER SERIES NO I278 / DECEMBER 20I0}

SHOCKING STUFF

\section{TECHNOLOGY, HOURS, AND FACTOR SUBSTITUTION '}

by Cristiano Cantore, ${ }^{2}$

Miguel A. León-Ledesma, ${ }^{3}$

Peter McAdam ${ }^{4}$ and

Alpo Willman ${ }^{5}$

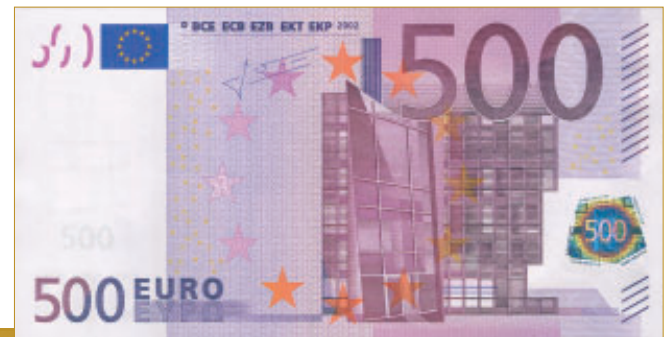

In 2010 all ECB

publication

feature a motif

taken from the

$€ 500$ banknote.

NOTE: This Working Paper should not be reported as representing the views of the European Central Bank (ECB).

The views expressed are those of the authors and do not necessarily reflect those of the ECB.

This paper can be downloaded without charge from http://www.ecb.europa.eu or from the Social Science Research Network electronic library at http://ssrn.com/abstract_id=1722035.

I We thank seminar participants at Aix-Marseille, Birkbeck, Cardiff, Goethe, Kent, Loughborough, Manchester, Oxford, Pablo de Olavide, Surrey, the Bank of Norway and Banco de España; the 2009 ASSET, CDMA, EEA, MMF, SAEe and 2010 CEF conferences; as well as Pierre-Richard Agénor, Yunus Aksoy, Susanto Basu, John Driffill, Martin Ellison, Jordi Gali, Paolo Guarda (discussant), Juan Francisco Jimeno, Michel Juillard, Paul Levine, Patrick Minford, Alessio Moro, Rachel Ngai, Gabriel Pérez-Quiros, Sergio Rebelo, Mathan Satchi, Katsuyuki Shibayama, Ron Smith and Michael Wickens for helpful discussions. McAdam is also visiting professor at the University of Surrey. 2 Department of Economics, University of Surrey, Surrey GU2 7XH, UK; e-mail: c.cantore@surrey.ac.uk

3 Department of Economics, University of Kent, Kent CT2 7NP, UK; e-mail: m.a.leonledesma@ kent.ac.uk 4 Research Dept., European Central Bank, Kaiserstrasse 29, D-603II Frankfurt am Main, Germany; e-mail: peter.mcadam@ecb.europa.eu 5 Research Dept., European Central Bank, Kaiserstrasse 29, D-603II Frankfurt am Main, Germany; 
(C) European Central Bank, 2010

Address

Kaiserstrasse 29

60311 Frankfurt am Main, Germany

Postal address

Postfach 160319

60066 Frankfurt am Main, Germany

Telephone

+496913440

Internet

http://www.ecb.europa.eu

Fax

+496913446000

All rights reserved.

Any reproduction publication and reprint in the form of a differen publication, whether printed or produced electronically, in whole or in part, is permitted only with the explicit written authorisation of the ECB or the authors.

Information on all of the papers published in the ECB Working Paper Series can be found on the ECB's website, http://www. ecb.europa.eu/pub/scientific/wps/date/ html/index.en.htm

ISSN 1725-2806 (online) 


\section{CONTENTS}

Abstract

Non-technical summary

1 Introduction

2 The normalized CES production function

2.1 Normalization

2.2 CES production and

factor-augmenting technology

3 The real business cycle model

4 The new Keynesian model

4.1 Households

4.2 Firms

4.3 Monetary authority

4.4 Aggregation and equilibrium

5 Calibration and impulse-response analysis

6 Technology and hours: threshold conditions

6.1 The general hours-technology rule

6.2 The simple hours-technology rule

7 Hours and technology: a labor demand and supply interpretation

7.1 Technology shocks and hours demand

7.2 Technology shocks and hours supply

7.3 A graphical analysis

7.4 Labor demand and supply in the NK model

8 Some robustness analysis

9 A time-varying correlation?

10 Conclusions

References

Appendices 


\begin{abstract}
The reaction of hours worked to technology shocks represents a key controversy between RBC and New Keynesian explanations of the business cycle. It sparked a large empirical literature with contrasting results. We demonstrate that, with a more general and data coherent supply and production framework ("normalized" factor-augmenting CES technology), both models can plausibly generate impacts of either sign. We develop analytical expressions to establish the threshold between positive and negative contemporaneous correlations for both models. These will crucially depend on the factor-augmentation nature of the shock, the elasticity of factor substitution, the capital income share, and the reaction of consumption. The impact of technology on hours can thus hardly be taken as evidence in support of any particular business-cycle model. Our results are also important as: i) we introduce the concept of normalization for DSGE models and, ii) they may help interpret possible time-variation in technology and hours correlations over time.
\end{abstract}

JEL Classification: E32, E23, E25.

Keywords: Technology Shocks, Hours Worked, RBC and NK models, Normalization, Factor Substitution, Factor Bias. 


\section{Non-technical Summary}

A key question for understanding the sources of business cycle fluctuations is whether the impact of a productivity shock is expansionary or contractionary for employment in the short-run. The standard tenet has it that, in flexible price models of fluctuations (RBC), productivity changes (driven by technology shocks) increase employment (hours worked) on impact. On the other hand, models with price rigidities (NK) imply that technology shocks reduce hours on impact as firms cannot accommodate the excess output by reducing prices. This dichotomy generated a large empirical literature with contrasting results. Researchers try to identify technology shocks and then analyze their correlation with hours worked in the short-run to discriminate between business cycle models.

Practically all models in both the RBC and NK variants assume that, in the face of a change in relative factor productivity (and factor prices) the economy substitutes capital and labor one for one, as is the case in the well known CobbDouglas production function. However, the existing evidence emphatically rejects this assumption: the elasticity of substitution between capital and labor is substantially less than one, making capital and labor gross complements. We thus argue that it is not satisfactory to use Cobb-Douglas in macroeconomic models and propose the introduction of a more flexible Constant Elasticity of Substitution (CES) production function where factor substitution can range from zero to infinity. This introduces the possibility that technology shocks are non-neutral: in the short-run shocks may "favor" some factors in the sense that their relative marginal product and their share in the economy's income increases. Hence, we re-state a widely used result in growth theory that technology shocks can be either capital or labor saving.

We then analyze the impact of technology shocks on hours worked using this more general and data coherent supply and production framework. We demonstrate that introducing CES supply with differing substitution possibility requires "normalization" for the calibration of dynamic general equilibrium models, and propose a simple yet robust way of calibrating the normalized supply side. We then show that, in the standard RBC model, capital-augmenting shocks yield positive hours responses, whilst labor-augmenting shocks can lead to either response sign. In the standard NK model, however, labor-augmenting shocks yield negative responses and capital-augmenting shocks were ambiguous. We conclude that the impact of technology shocks on hours worked can hardly be taken as evidence in support of any particular business-cycle model.

More generally, we derived threshold rules for the determination of the technologyhours sign on impact. In each model and shock case, we showed that there exists some factor substitution value whereby a given technology-hours impact changed sign. The key margins - other than the substitution elasticity itself and the factor bias of technology shocks - are the capital share, risk aversion, and the marginal propensity to consume. The latter essentially boils down to how persistent shocks 
are and the strength of investment adjustment costs. These margins are then interpreted using a simple labor supply and demand framework.

Our threshold rules may help shed light on possible time-variation in the hourstechnology correlation. This is potentially a useful insight since much of the literature appears motivated by concerns of statistical fit rather than uncovering the underlying economic mechanisms. In our analysis, time variations may result from (i) time variation in the relative intensity of labor and capital augmenting shocks and (ii) time-variations in parameters (the substitution elasticity being prime among them).

Our analysis may also open important new avenues for research. For instance, if it is not satisfactory for business-cycle models to assume unitary factor substitution, the practice implemented here of appropriately normalizing the supply side for calibration of DSGE models should become standard and related sensitivities analyzed. Moreover, although theoretical, our results can have empirical implications. We might, for instance, be able to exploit changes in factor income shares to identify different sorts of technology shocks in modeling and SVAR analysis. The models presented here can also serve as a benchmark to study the business cycle properties of movements in factor shares. 


\section{Introduction}

The reaction of hours worked to a technology shock has been a key controversy in macroeconomics over the last decade. According to the standard real business cycle (RBC) model, hours worked should rise after a (positive) productivity shock. However, in an influential paper, Galí (1999), using a structural VAR (SVAR) with long-run restrictions, found the impact to be negative. This evidence has since been interpreted as favoring the New-Keynesian (NK) sticky-price model of business-cycle fluctuations. ${ }^{1}$

Subsequent literature was mostly supportive of a negative correlation between technology and hours. ${ }^{2}$ Christiano, Eichenbaum and Vigfusson (2003), though, challenged these results arguing that they were driven by the way researchers treat hours worked; using hours on a per capita basis, they found a positive hourstechnology short run correlation. ${ }^{3}$

Econometric identification, hence, took center stage in the debate. Fernald (2007) emphasized the importance of low-frequency trend-breaks in productivity and found a negative but time-varying impact of technology on hours. Dedola and Neri (2007) use sign restrictions for VAR identification and found that hours worked are likely to increase. Uhlig (2004), using a medium-run identification scheme, also finds support for a mildly positive impact. Pesavento and Rossi (2005), use an agnostic method that does not require choosing between a specification in levels or in first differences, found that hours fall after a technology shock, but that the effect is short lived. Chari, Kehoe and McGrattan (2008), however, maintain that SVAR models are incapable of distinguishing between different explanations of the business cycle (i.e. RBC vs. sticky prices). They argue that, if non-technology shocks account for an important part of business-cycle fluctuations, SVAR models can erroneously favor a NK model when the data has been generated using an RBC model. The method proposed in the influential paper by Basu, Fernald and Kimball (2006), though, is free from the shortcomings of the SVAR identification restrictions. They derive a measure of aggregate technology change from sectoral-level data and found technological change and factor inputs to be negatively correlated. They conclude that technology improvements

\footnotetext{
${ }^{1}$ It is well known that a standard RBC model could also generate a negative technology-hours response if the coefficient of relative risk aversion is sufficiently high (above unity). Rotemberg (2003) also showed that an RBC model with protracted technical diffusion could generate a negative technology-hours correlation. In the NK case, the presence of various rigidities (the most popular of which is price staggering) tends to imply that aggregate demand grows by less than the growth in technology prompting an initial reduction in employment. See Galí (2008) and Galí and Rabanal (2004) for thorough discussions. Fernández-Villaverde (2010) and Tovar (2008) provide effective discussions and analysis of modern DSGE models.

${ }^{2}$ E.g., Francis, Owyang and Theodorou (2003) and the references therein.

${ }^{3}$ See Whelan (2009) for a further discussion of the Galí (1999)-Christiano, Eichenbaum and Vigfusson (2003) debate.
} 
are contractionary on impact. Finally, recent work is suggestive of a time-varying correlation, e.g., Galí and Gambetti (2009). ${ }^{4}$

The effect of technical change on employment is, in fact, a long-standing debate in economics - see, for example Wicksell (1911)'s discussion of the historical "machinery question". The traditional Ricardian effect - defended by Hicks (1969) - supported the idea that technological advancement reduces employment in the short run, but increases it in the long run. ${ }^{5}$ The kind of mechanism envisaged, however, did not rest on the introduction of nominal rigidities that characterizes much of modern macroeconomics. It relied on aspects of the production process such as the degree to which different factors substitute or complement one another, and the extent to which technical change is non neutral.

Modern business cycle models, though, have generally abstracted from these aspects. ${ }^{6}$ They tend to impose aggregate (unitary elasticity) Cobb-Douglas production which is both highly restrictive and uninformative regarding biases in technical change. The choice may be considered startling given the avowed interest of the literature in promoting (or testing) the cyclical importance of "technology" shocks. In that light, our work may be considered as synthesizing developments in production/growth theory - where non-unitary substitution elasticities and factoraugmenting technology shocks are relied upon to describe various economic phenomena (e.g., Acemoglu (2009)) - with developments in dynamic stochastic general equilibrium models used to explain business cycle patterns and inform policy.

Indeed, there is now mounting evidence that aggregate production may be better characterized by a non-unitary substitution elasticity; the evidence in Klump, McAdam and Willman (2007) and Chirinko (2008) suggests 0.4-0.6 as a benchmark range for the US. Along these lines, Jones $(2003,2005)$ argued that capital shares exhibit such protracted swings and trends in many countries as to be inconsistent with Cobb-Douglas (see also Blanchard (1997), McAdam and Willman (2008)). ${ }^{7}$ Moreover, as Acemoglu (2009, chap. 15) points out, there is little reason

\footnotetext{
${ }^{4}$ Alexopoulos (2010) pursues a novel approach: using an index of information technology publications, she finds that output and (albeit to a small extent) employment rise following a technology shock.

${ }^{5}$ This is in contrast to Marxian theories that supported the existence of permanent (negative) effects on employment (see Beach (1971) for a discussion).

${ }^{6}$ Francis and Ramey (2005) can be credited with noting that hours fall in response to a labor-saving technology shock in an RBC model in the limit case of (albeit non-normalized) Leontief technology. Although at business-cycle frequencies low substitution elasticities might be expected, zero factor substitution is a strong assumption with the counter-factual implication that shares of capital and labor in output approach one-half. Furthermore, in the technology and growth literature, the Leontief form is usually ruled out given its dis-equilibrium implications for growth and optimal savings, e.g., Barro and Sala-i-Martin (2004).

${ }^{7}$ Jones $(2003,2005)$ model provides an explanation for the existence of an aggregate production function that exhibits a (far) less than unitary substitution elasticity over business-cycle frequencies but asymptotes to Cobb-Douglas in the long-run.
} 
to suppose that, over business-cycle frequencies, technical change will be neutral or embody balanced growth: technical change typically benefits some factors and some agents more than others.

When investigating the ramifications of a non-unitary substitution elasticity and factor-augmenting technology shocks in dynamic macro-models one necessarily faces the issue of normalization (following the seminal contribution by La Grandville (1989b) and subsequent work by Klump and de La Grandville (2000) and León-Ledesma, McAdam and Willman (2010)). This is an aspect that is not yet widely known, and certainly not known in the DSGE context. Normalization essentially implies representing the supply side of the model (i.e., production function and factor demands) in consistent indexed number form. In our context, normalization turns out to be absolutely crucial to ensure the validity of comparative statics, and for meaningful and consistent calibration of the deep parameters of the supply side of the model. ${ }^{8}$

Accordingly, while remaining agnostic about empirical identification methods, we generalize the supply side of both standard RBC and NK models. In doing so, we demonstrate that both models can yield positive or negative responses of hours worked to technology shocks. Importantly, these responses are also empirically plausible given the existing estimates of the relevant parameters. We further derive threshold rules for the sign of this response. We explain the intuition behind these rules and carry out a comprehensive robustness analysis. This threshold rule may further help interpret the time variation in technology-hours correlations that some researchers report.

The paper is organized as follows. The next section discusses the importance of normalization alongside biased technical change in the more general Constant Elasticity of Substitution (CES) production function. Sections 3 and 4 present the RBC and NK models. Section 5 discusses the calibration and presents some key simulation results. Section 6 derives threshold rules (general and simplified) determining the sign of the response of hours to technology shocks. Section 7 interprets that rule in a more intuitive context through the lens of shifts in labor demand and supply. Section 8 presents some robustness analysis. Section 9 discusses possible time variation in the technology-hours impact. Finally, section 10 concludes. The paper is complemented by a technical appendix where we present extensive numerical analysis, robustness analysis, and the derivation of the threshold rules under different types of preferences.

\footnotetext{
${ }^{8}$ As far as we know, ours is the first attempt to incorporate and consistently implement normalized supply into fully-fledged dynamic stochastic general equilibrium models.
} 


\section{The Normalized CES Production Function}

The technology assumption adopted by modern business cycle models has almost exclusively been Cobb Douglas. This functional form constrains the substitution elasticity between factors of production to unity and is unable to separately identify capital and labor augmenting technology shocks. The more general CES production function, by contrast, nests Cobb-Douglas as a special case and admits the possibility of neutral and non-neutral productivity changes.

\subsection{Normalization}

At a simple level, one can think of normalization as removing the problem that arises from the fact that labor and capital are measured in different units - although its importance goes well beyond that. Under Cobb-Douglas, normalization plays no role since, due to its multiplicative form, differences in units are absorbed by the scaling constant. The CES function, by contrast, is highly non-linear, and so, unless correctly normalized, out of its three key parameters - the efficiency parameter, the distribution parameter and the substitution elasticity - only the latter is deep. The other two parameters turn out to be affected by the size of the substitution elasticity and factor income shares. Accordingly: i) if one is interested in model sensitivity with respect to production parameters (as here), normalization is essential to have interpretable comparisons; and ii) without a proper normalization, nothing ensures that factor shares equal the distribution parameter, hence invalidating inference based on impulse-response functions (IRFs).

Let us start with the general definition of a linear homogenous production function:

$$
Y_{t}=F\left(\Gamma_{t}^{K} K_{t}, \Gamma_{t}^{H} H_{t}\right)=\Gamma_{t}^{H} H_{t} f\left(\kappa_{t}\right)
$$

where $Y_{t}$ is output, $K_{t}$ capital and $H_{t}$ the labor input. The terms $\Gamma_{t}^{K}$ and $\Gamma_{t}^{H}$ capture capital and labor-augmenting technical progress, respectively. To circumvent problems related to the "Diamond-McFadden impossibility theorem", ${ }^{9}$ researchers usually assume specific functional forms for these functions, e.g., $\Gamma_{t}^{j}=\Gamma_{0}^{j} e^{z_{t}^{j}}$ where $z_{t}^{j}$ can be a stochastic or deterministic technical progress function associated to factor $i$.

The case where $z_{t}^{K}=z_{t}^{H}>0$ denotes Hicks-Neutral technology; $z_{t}^{K}>0$, $z_{t}^{H}=0$ yields Solow-Neutrality; $z_{t}^{K}=0, z_{t}^{H}>0$ represents Harrod-Neutrality; and $z_{t}^{K}>0 \neq z_{t}^{H}>0$ indicates general factor-augmentation. The term $\kappa_{t}=$ $\left(\Gamma_{t}^{K} K_{t}\right) /\left(\Gamma_{t}^{H} H_{t}\right)$ is the capital-labor ratio in efficiency units. Likewise define $\varphi_{t}=y_{t} /\left(\Gamma_{t}^{H} H_{t}\right)$ as per-capita production in efficiency units.

\footnotetext{
${ }^{9}$ See Diamond and McFadden (1965), Diamond, McFadden and Rodriguez (1978).
} 
The elasticity of substitution can then be expressed as:

$$
\sigma=-\frac{f^{\prime}(\kappa)\left[f(\kappa)-\kappa f^{\prime}(\kappa)\right]}{\kappa f(\kappa) f^{\prime \prime}(\kappa)} \in[0, \infty]
$$

This definition can be viewed as a second-order differential equation in $\kappa$ having the following general CES production function as its solution:

$$
\varphi_{t}=a\left[\kappa_{t}^{\frac{\sigma-1}{\sigma}}+b\right]^{\frac{\sigma}{\sigma-1}} \Rightarrow Y_{t}=a\left[\left(\Gamma_{t}^{K} K_{t}\right)^{\frac{\sigma-1}{\sigma}}+b\left(\Gamma_{t}^{H} H_{t}\right)^{\frac{\sigma-1}{\sigma}}\right]^{\frac{\sigma}{\sigma-1}}
$$

where $a$ and $b$ are two arbitrary constants of integration with the following correspondence with the original Arrow et al. (1961) non-normalized form, which, after some rearrangements can be presented in conventional form:

$$
Y_{t}=\mathbb{J}\left[\alpha\left(\Gamma_{t}^{K} K_{t}\right)^{\frac{\sigma-1}{\sigma}}+(1-\alpha)\left(\Gamma_{t}^{H} H_{t}\right)^{\frac{\sigma-1}{\sigma}}\right]^{\frac{\sigma}{\sigma-1}}
$$

where efficiency parameter $\mathbb{J}=a(1+b)^{\frac{\sigma}{\sigma-1}}$ and distribution parameter $\alpha=$ $1 /(1+b)<1$.

An economically meaningful identification of these integration constants $a$ and $b$ (and further $\mathbb{J}$ and $\alpha$ ) is given by the fact that $\sigma$ is a point elasticity relying on three baseline (or "normalized", $t=0$ ) values: a given capital intensity $\kappa_{0}=$ $\Gamma_{0}^{K} K_{0} /\left(\Gamma_{0}^{H} H_{0}\right)$, a given marginal rate of substitution, $\frac{\partial\left(Y_{0} / H_{0}\right)}{\partial\left(Y_{0} / K_{0}\right)}$, and a given level of per-capita production $\varphi_{0}=Y_{0} /\left(\Gamma_{0}^{H} H_{0}\right)$. For simplicity, and without loss of generality, we scale the components of technical progress such that $\Gamma_{0}^{K}=\Gamma_{0}^{H}=1$. Accordingly, we can transform (4) into the key normalized form,

$$
Y_{t}=Y_{0}\left[\alpha_{0}\left(\frac{K_{t}}{K_{0}} \Gamma_{t}^{K}\right)^{\frac{\sigma-1}{\sigma}}+\left(1-\alpha_{0}\right)\left(\frac{H_{t}}{H_{0}} \Gamma_{t}^{H}\right)^{\frac{\sigma-1}{\sigma}}\right]^{\frac{\sigma}{\sigma-1}}
$$

where distribution parameter $\alpha_{0}=r_{0} K_{0} /\left(r_{0} K_{0}+w_{0} H_{0}\right)$ has a clear economic interpretation: the capital income share evaluated at the point of normalization. We see that all parameters of (5) are deep, demonstrated by the fact that at the point of normalization, the left-hand-side equals the right-hand side for all values of $\sigma, \alpha_{0}$ and the parameterization of $\Gamma_{t}^{K}$ and $\Gamma_{t}^{H}$.

By contrast, comparing (4) with (5), the parameters of the non-normalized function depend on the normalized value of the factors and the factor returns as 
well as on the $\sigma$ value itself:

$$
\begin{aligned}
& \mathbb{J}(\sigma, \cdot)=Y_{0}\left[\frac{r_{0} K_{0}^{1 / \sigma}+w_{0} H_{0}^{1 / \sigma}}{r_{0} K_{0}+w_{0} H_{0}}\right]^{\frac{\sigma}{\sigma-1}} \\
& \alpha(\sigma, \cdot)=\frac{r_{0} K_{0}^{1 / \sigma}}{r_{0} K_{0}^{1 / \sigma}+w_{0} H_{0}^{1 / \sigma}} .
\end{aligned}
$$

Accordingly, in the non-normalized formulation, parameters $\mathbb{J}$ and $\alpha$ have no theoretical or empirical meaning. Hence, varying $\sigma$, whilst holding $\mathbb{I}$ and $\alpha$ constant, is inconsistent for comparative-static purposes. Each of the resulting CES functions goes through different fixed points and we can say that each resulting CES function belongs to "different families", La Grandville (2009).

Since, in the non-normalized case, parameter $\alpha$ depends on the point of normalization as well as on $\sigma$, it is obvious that the dynamic responses to shocks can change as we vary $\sigma$, since the elasticity of output with respect to capital and labor will change. This will, as we shall see later, turn out to be a crucial parameter for the determination of the response of hours. That is, by changing $\sigma$ we would also be changing the capital and labor intensities. Also, parameter $\alpha$ in a nonnormalized CES will not match the capital share. Hence, in a calibrated dynamic simulation we would not be controlling for this important parameter, rendering IRFs invalid. In our general equilibrium setting, we are interested in the dynamic responses of variables in a stationary model. Hence, we need to ensure that factor shares in steady state (the initial and end point of our simulations) are constant and equal to $\alpha_{0}$ and $1-\alpha_{0}$. Also, output, capital, labor, consumption and factor payments are common at this point for different $\sigma$ 's. We hence choose to make the steady state our normalization point.

A logical way to proceed is then to choose a steady state and then calibrate the model using this as the normalization point. We can, for instance, set $Y_{0}$ and $H_{0}$ to 1 . Since the real interest rate is determined by preferences and depreciation, we can then, given the income/factor income identity,

$$
\underbrace{Y_{0}}_{=1} \equiv \underbrace{r_{0} K_{0}}_{\alpha_{0}}+\underbrace{w_{0} H_{0}}_{1-\alpha_{0}}
$$

define the steady-state capital stock as $K^{*}=\alpha_{0} / r_{0}$, where $\alpha_{0}$ and $r_{0}$ are the capital income share and real interest rate at the chosen steady-state. The real normalized/steady-state wage is solved as $w^{*}=1-\alpha_{0}$. This ensures that the model is consistent, so factor shares sum to one and consumption plus investment equals output. 


\subsection{CES Production and Factor-Augmenting Technology}

The CES production function, (4) or (5), nests Cobb-Douglas when $\sigma \rightarrow 1$; the Leontief function (i.e., fixed factor proportions) when $\sigma \rightarrow 0$; and a linear production function (i.e., perfect factor substitutes) when $\sigma \rightarrow \infty$. Although factors are always substitutes, the higher is $\sigma$ the greater the similarity between capital and labor. Thus, when $\sigma<1$, we say that factors are gross complements in production and gross substitutes when $\sigma>1$ (La Grandville (1989a), Acemoglu (2002)).

In business-cycle models factor substitutability and non-neutral technical change will matter in so far as they influence developments in output, relative prices, factor intensities, income shares and cost pressures. Movements in these variables affect the inter-temporal decisions of consumers and firms. Some indications of the key role played by factor substitution can be gauged from the following.

Assuming competitive markets and profit maximization, relative factor income shares and relative marginal products are (dropping time subscripts and abstracting from normalization):

$$
\begin{aligned}
& \Theta=\frac{r K}{w H}=\frac{\alpha}{1-\alpha}\left(\frac{\Gamma^{K} K}{\Gamma^{N} H}\right)^{\frac{\sigma-1}{\sigma}} \\
& \iota=\frac{F_{K}}{F_{H}}=\frac{\alpha}{1-\alpha}\left[\left(\frac{K}{H}\right)^{-\frac{1}{\sigma}}\left(\frac{\Gamma^{K}}{\Gamma^{H}}\right)^{\frac{\sigma-1}{\sigma}}\right]
\end{aligned}
$$

It is straightforward to show that the effect of technical bias and capital deepening on factor income shares and relative factor prices is related to whether factors are gross complements or gross substitutes:

$$
\operatorname{sign}\left\{\frac{\partial \iota}{\partial\left(\Gamma^{K} / \Gamma^{H}\right)}\right\}, \operatorname{sign}\left\{\frac{\partial \Theta}{\partial(K / H)}\right\}, \operatorname{sign}\left\{\frac{\partial \Theta}{\partial\left(\Gamma^{K} / \Gamma^{H}\right)}\right\}=\operatorname{sign}\{\sigma-1\}
$$

Hence, an increase in factor $J$-augmenting $(J=K, H)$ technical change "favors" factor $J$ (i.e., implying $F_{j}>F_{i \neq j}$ and raising $J$ 's income share for given factor proportions) if factors are gross substitutes $(\sigma>1)$. The effects reverse if factors are gross complements. Thus, it is only in the gross-substitutes case that a factor $J$-augmenting change in technology is $J$-biased. Naturally, the relations between the substitution elasticity, technical bias and factor shares evaporate under CobbDouglas: factor income shares are time-invariant and relative factor prices are purely determined by capital deepening.

Equations (9)-(11) illustrate the importance of factor substitution and technical bias. Namely that the impact of technology shocks on factor payments depends on the substitution elasticity and the factor bias of the shock. This influences the 
dynamic response of interest and wages (and hence hours) to technology shocks.

Note, though, statement (11) defines factor demand reactions to technology changes. They therefore abstract from labor-supply reactions. However, our analysis - in the following sections - suggests that the conclusions concerning factor shares do not change with endogenous labor supply. Although the corresponding conclusion concerning relative factor prices $-F_{j}>F_{i}$ - need no longer hold.

\section{The Real Business Cycle model}

The standard RBC model is a variant of the representative agent neoclassical model, where business cycles are due to non-monetary sources (primarily, changes in technology). The model is well known and can therefore be introduced compactly. The standard model with CES production technology in the supply side is given by (for expositional simplicity we omit the expectations operator):

$$
\begin{gathered}
C_{t}^{-\sigma_{c}}=\beta C_{t+1}^{-\sigma_{c}}\left[1+r_{t+1}-\delta\right] \\
w_{t}=v H_{t}^{\gamma} C_{t}^{\sigma_{c}} \\
Y_{t}=C E S_{t}=Y_{0} e^{z_{t}^{\boxplus}}\left[\alpha_{0}\left(\frac{K_{t-1}}{K_{0}} e^{z_{t}^{K}}\right)^{\frac{\sigma-1}{\sigma}}+\left(1-\alpha_{0}\right)\left(\frac{H_{t}}{H_{0}} e^{z_{t}^{H}}\right)^{\frac{\sigma-1}{\sigma}}\right]^{\frac{\sigma}{\sigma-1}} \\
w_{t}=\left(1-\alpha_{0}\right)\left(\frac{Y_{0}}{H_{0}} e^{z_{t}^{\sharp}} e^{z_{t}^{H}}\right)^{\frac{\sigma-1}{\sigma}}\left(\frac{Y_{t}}{H_{t}}\right)^{\frac{1}{\sigma}} \\
r_{t}=\alpha_{0}\left(\frac{Y_{0}}{K_{0}} e^{z_{t}^{\sharp}} e^{z_{t}^{K}}\right)^{\frac{\sigma-1}{\sigma}}\left(\frac{Y_{t}}{K_{t-1}}\right)^{\frac{1}{\sigma}} \\
C_{t}+K_{t}-(1-\delta) K_{t-1} \leq Y_{t} \\
z_{t}^{j}=\rho z_{t-1}^{j}+\varepsilon_{t}^{j}
\end{gathered}
$$

where $C_{t}, w_{t}$ and $r_{t}$ are, respectively, real consumption, real wages and the real interest rate. Parameters $\beta, \delta$ and $v$ represent, respectively, the discount factor, the capital depreciation rate and a scaling constant. $z_{t}^{j}$ are technology shocks for $j=K, H, \mathbb{H}$ (i.e., capital-augmenting, labor-augmenting, and Hicks-neutral shocks respectively). Equations (12) and (13) represent the household's optimal 
consumption and labor supply choices given the following utility function, ${ }^{10}$

$$
U_{t}=\frac{C_{t}^{1-\sigma_{c}}}{1-\sigma_{c}}-v \frac{H_{t}^{1+\gamma}}{1+\gamma}
$$

where $\sigma_{c}$ is the coefficient of relative risk aversion and $\gamma$ is the inverse of the Frisch elasticity. Solving equation (13) for hours demonstrates that, after a shock, hours rise if real wages grow faster than consumption. Equations (14) to (16) are the CES production function and its factor derivatives in normalized form. Equation (17) is the resource constraint. Equation (18) shows that technology shocks are assumed to follow an $\mathrm{AR}(1)$ process.

The model could straightforwardly be expanded to contain monopolistic competition and investment adjustment costs as in the NK model discussed below. In the limiting case of fully-flexible prices the NK model would encompass that more generalized RBC version as a special case. The importance of these limiting cases comparisons becomes clear from section 6 onwards.

\section{The New Keynesian Model}

The NK model builds on the RBC framework with the addition of monopolistic competition, nominal rigidities, investment adjustment costs and a monetary policy rule.

\subsection{Households}

As before, the representative household maximizes utility function, (19) supplemented by (separable) real money balances $m_{t}$,

$$
U_{t}=\frac{C_{t}^{1-\sigma_{c}}}{1-\sigma_{c}}-v \frac{H_{t}^{1+\gamma}}{1+\gamma}+\digamma\left(m_{t}\right)
$$

with $\digamma^{\prime}>0, \digamma^{\prime \prime}<0$. The consumption good is assumed to be a composite good produced with a continuum of differentiated goods, $C_{i, t}, i \in[0,1]$, via the aggregator function:

$$
C_{t}=\left[\int_{0}^{1} C_{i, t}^{1-\frac{1}{\eta}} d i\right]^{\frac{1}{1-\frac{1}{\eta}}}
$$

\footnotetext{
${ }^{10}$ We employ separable utility which makes our analysis more general. Non-separable preferences are examined in Appendix B.
} 
where $\eta$ represents the intra-temporal elasticity of substitution across different varieties of consumption goods. To find total consumption demand for each variety $i$, one can solve the standard problem of minimizing total cost subject to (21), which yields the downward sloped demand function:

$$
C_{i t}=\left(\frac{P_{i t}}{P_{t}}\right)^{-\eta} C_{t}
$$

where $P_{t}$ is the nominal price index given by:

$$
P_{t}=\left[\int_{0}^{1} P_{i t}^{1-\eta} d i\right]^{\frac{1}{1-\eta}}
$$

Households are assumed to have access to a complete set of nominal statecontingent assets. The household maximizes (19) subject to a sequence of flow budget constraints given by,

$$
q_{t} b_{t}+m_{t}+C_{t}+I_{t}=\frac{b_{t-1}+m_{t-1}}{\pi_{t}}+w_{t} H_{t}+r_{t}^{K} K_{t-1}+\Pi_{t}
$$

where $b_{t}$ represents the quantity of one-period nominally riskless discount bonds (or any one-period claim) purchased (or issued) in period $t$ and maturing in period $t+1$. Each bond pays one unit of money at maturity and its price is $q_{t}$. Because $1-q_{t}$ corresponds to the nominal loss of purchasing one unit of money instead of purchasing bonds the equality $q_{t}=R_{t}^{-1}$ must hold, where $R_{t}$ is the gross nominal interest rate. Variable $I_{t}$ is investment, $r_{t}^{K}$ the rental price of the capital stock, $\pi_{t}=\frac{P_{t}}{P_{t-1}}$ is the gross inflation rate and $\Pi_{t}$ denotes profits received from the ownership of firms.

The resource constraint is,

$$
C_{t}+I_{t} \leq Y_{t}
$$

and capital accumulation is given by,

$$
K_{t}=(1-\delta) K_{t-1}+I_{t}\left[1-\frac{\psi}{2}\left(\frac{I_{t}}{I_{t-1}}-1\right)^{2}\right]
$$

Changes in the capital stock are thus assumed to be subject to a convex adjustment cost with $\psi>0$.

We assume the investment good $I_{t}$ to be a composite made of the aggregator function type (21). Hence, investment demand for each variety has the same form as the consumption function: $I_{i t}=\left(P_{i t} / P_{t}\right)^{-\eta} I_{t}$. 
The household chooses $C_{t}, b_{t}, H_{t}, K_{t}, I_{t}$, and $m_{t}$ to maximize utility (19) subject to (24), (26) and the no-Ponzi-game constraint. Letting $\lambda_{t}$ and $\lambda_{t} q_{t}$ denote the Lagrange multipliers associated with constraints (24) and (26), respectively, yields,

$$
\sum_{t=0}^{\infty} \beta^{t}\left\{\begin{array}{c}
{\left[\frac{C_{t}^{1-\sigma_{c}}}{1-\sigma_{c}}-v \frac{H_{t}^{1+\gamma}}{1+\gamma}+\digamma\left(m_{t}\right)\right]} \\
+\lambda_{t}\left[w_{t} H_{t}+r_{t}^{K} K_{t-1}-C_{t}-I_{t}-R_{t}^{-1} b_{t}-m_{t}+\frac{b_{t-1}+m_{t-1}}{\pi_{t}}+\Pi_{t}\right] \\
+\lambda_{t} q_{t}\left[(1-\delta) K_{t-1}+I_{t}\left(1-\frac{\psi}{2}\left(\frac{I_{t}}{I_{t-1}}-1\right)^{2}\right)-K_{t}\right]
\end{array}\right\}
$$

The first-order conditions are:

$$
\begin{gathered}
C_{t}^{-\sigma_{c}}=\lambda_{t} \\
\lambda_{t}=\beta R_{t} \frac{\lambda_{t+1}}{\pi_{t+1}} \\
w_{t}=v \frac{H_{t}^{\gamma}}{\lambda_{t}} \\
\lambda_{t} q_{t}=\beta \lambda_{t+1}\left[r_{t+1}^{k}+(1-\delta) q_{t+1}\right] \\
+\beta \psi \lambda_{t+1} q_{t+1}\left(\frac{I_{t+1}}{I_{t}}\right)^{2}\left(\frac{I_{t+1}}{I_{t}}-1\right) \\
\left.\left.\digamma^{\prime}=\lambda_{t}-\beta \frac{I_{t+1}}{I_{t+1}}-1\right)^{2}-\psi \frac{I_{t}}{I_{t-1}}\left(\frac{I_{t}}{I_{t-1}}-1\right)\right]
\end{gathered}
$$

The last condition determines the demand for money function. However, in the current framework, with a Taylor-rule based monetary policy (described below), money demand is purely recursive.

\subsection{Firms}

We assume that single firms operating in a monopolistically competitive environment and produce one good of variety $i$. The firm does so by using capital and labor following a production technology:

$$
e^{z_{t}^{\sharp}} F\left(e^{z_{t}^{K}} K_{i t}, e^{z_{t}^{H}} H_{i t}\right)-\chi
$$


where the $F$ is the same normalized CES production function presented before and $\chi$ represents fixed costs in production. ${ }^{11}$ Given the consumption and investment demand functions, aggregate demand for good $i$ will then be given by:

$$
Y_{i t}=\left(\frac{P_{i t}}{P_{t}}\right)^{-\eta} Y_{t}
$$

Real profits for firm $i$ expressed in terms of the composite good are,

$$
\Pi_{i t}=\frac{P_{i t}}{P_{t}} Y_{i t}-r_{t}^{K} K_{i t-1}-w_{t} H_{i t}
$$

We assume, as usual in this literature, that firms rent labor and capital services from a centralized market and that capital input can be readily reallocated across industries. The objective of the firm is to choose the plan $H_{i t}, K_{i t-1}$ and $p_{i t}$, so as to maximize the present discounted value of the profit stream assuming that the firm satisfies demand at the posted price subject to the production function constraint. Hence,

$$
\sum_{s=t}^{\infty} R_{t, s}^{-1} P_{s}\left\{\Pi_{i s}+m c_{i s}\left[e^{z_{t}^{H}} F\left(e^{z_{t}^{K}} K_{i t-1}, e^{z_{t}^{H}} H_{i t}\right)-\chi-Y_{i s}\right]\right\}
$$

where $R_{t, s}=\prod_{\tau=t}^{s} R_{\tau}$ with $R_{t, t}=1$ and $R_{t, t+1}=R_{t}$. Variable $m c_{i t}$ is the Lagrange multiplier related to the production function constraint. We derive the following cost-minimization conditions with respect to labor and capital:

$$
\begin{aligned}
& m c_{i t} e^{z_{t}^{\boxplus}} F_{H}\left(e^{z_{t}^{K}} K_{i t-1}, e^{z_{t}^{H}} H_{i t}\right)=w_{t} \\
& m c_{i t} e^{z_{t}^{\sharp}} F_{K}\left(e^{z_{t}^{K}} K_{i t-1}, e^{z_{t}^{H}} H_{i t}\right)=r_{t}^{K}
\end{aligned}
$$

We see that the Lagrange multiplier $m c_{i t}$ has a clear economic interpretation, i.e. the real marginal cost of the firm. It is also straightforward to show that $m c_{i t}$ is the same for all firms $i$. The ratio of (38) and (39) imply that $\frac{F_{K}\left(K_{i t-1}, H_{i, t}\right)}{F_{H}\left(K_{i, t-1}, H_{i, t}\right)}=\frac{r_{t}^{K}}{w_{t}}$, the right-hand-side of which is common to all firms. We know that the marginal productivities of labor and capital for any linearly homogeneous production function can be presented in terms of capital intensity $\frac{K_{i t-1}}{H_{t}}$ and common technical progress.

\footnotetext{
${ }^{11}$ These are chosen to ensure zero profits in steady state. This in turn guarantees that there is no incentive for other firms to enter the market in the long run. See Coenen, McAdam and Straub (2008).
} 
Now common input prices imply that capital intensities and hence also marginal productivities and marginal costs must be equal across firms, i.e. $m c_{i t}=m c_{t}$.

The second part of the firm's optimization problem is to set the optimal price level subject to Calvo pricing, where in each period the probability of re-optimizing is $1-\theta$ (for simplicity, we do not assume price indexation). Therefore, utilizing demand function (35), the Lagrangian (37) covering only those firms which are allowed to re-optimize their price level on period $t$, can be transformed into,

$$
\sum_{s=t}^{\infty} R_{t s}^{-1} P_{s} \theta^{s-t}\left\{\begin{array}{c}
\left(\frac{\tilde{P_{i t}}}{P_{s}}\right)^{1-\eta} Y_{s}-r_{t}^{K} K_{i t-1}-w_{t} H_{i t} \\
+m c_{s}\left[e^{z_{t}^{\boxplus !}} F\left(e^{z_{t}^{K}} K_{i t-1}, e^{z_{t}^{H}} H_{i t}\right)-\chi-\left(\frac{\tilde{P_{i t}}}{P_{s}}\right)^{-\eta} Y_{s}\right]
\end{array}\right\}
$$

where we denote the re-optimized price level by $\tilde{P}_{i t}$. Maximizing (40) with respect to $\tilde{P}_{i t}$ yields,

$$
\sum_{s=t}^{\infty} R_{t s}^{-1} \theta^{s-t}\left(\frac{\tilde{P}_{i t}}{P_{s}}\right)^{-1-\eta} Y_{s}\left(m c_{s}-\frac{\eta-1}{\eta} \frac{\tilde{P}_{i t}}{P_{s}}\right)=0
$$

This expression tells us that the optimal price equals a weighed sum of future expected mark-ups over marginal cost and that the re-optimized price level is same for all firms, i.e. $\widetilde{P}_{i t}=\widetilde{P}_{t}$. Utilizing (29), this condition can be expressed recursively, by introducing two auxiliary variables $x 1_{t}$ and $x 2_{t}$, as:

$$
\begin{gathered}
x 1_{t}=\widetilde{p}_{t}^{-1-\eta} Y_{t} m c_{t}+\theta \beta\left[\frac{\lambda_{t+1}}{\lambda_{t}} \pi_{t+1}^{\eta}\left(\frac{\widetilde{p}_{t}}{\widetilde{p}_{t+1}}\right)^{-1-\eta} x 1_{t+1}\right] \\
x 2_{t}=\widetilde{p}_{t}^{-\eta} Y_{t}+\theta \beta\left[\frac{\lambda_{t+1}}{\lambda_{t}} \pi_{t+1}^{\eta-1}\left(\frac{\widetilde{p}_{t}}{\widetilde{p}_{t+1}}\right)^{-\eta} x 2_{t+1}\right] \\
x 2_{t}=\frac{\eta}{\eta-1} x 1_{t}
\end{gathered}
$$

where $\widetilde{p}_{t}=\frac{\widetilde{P}_{t}}{P_{t}}$.

\subsection{Monetary authority}

The monetary authority follows a Taylor-type rule:

$$
\log \left(R_{t} / R\right)=\alpha_{r} \log \left(R_{t-1} / R\right)+\alpha_{\pi} \log \left(\pi_{t} / \pi\right)+\alpha_{y} \log \left(Y_{t} / Y_{t}^{f}\right)
$$


where $R_{t}$ denotes the nominal interest rate. Consistent with the DSGE model, potential output, $Y^{f}$, is defined as the level of output that would prevail under flexible prices and wages, e.g., Smets and Wouters (2007).

We also consider the responses of the economy under optimal (Ramsey) policy according to which the monetary authority sets the optimal path of all variables in the economy by maximizing agents' welfare subject to the relations describing the competitive economy, e.g., Levine, McAdam and Pearlman (2008). ${ }^{12}$

\subsection{Aggregation and Equilibrium}

Looking at expression (41) we see that all firms that are able to change their price in a given period chose the same price so we can drop subscript $i$ from the equilibrium conditions. By taking into account relative price dispersion across varieties, the resource constraint in this model is given by the following three expressions:

$$
\begin{gathered}
Y_{t}=\frac{1}{S_{t}}\left[e^{z_{t}^{\boxplus}} F(\cdot, t)-\chi\right] \\
Y_{t}=C_{t}+I_{t} \\
S_{t}=(1-\theta) \tilde{p}_{t}^{-\eta}+\theta \pi_{t}^{\eta} S_{t-1}
\end{gathered}
$$

where $S$ is a state variable that measures the resource costs induced by the inefficient price dispersion present in the Calvo problem in equilibrium. ${ }^{13}$

\section{$5 \quad$ Calibration and impulse-response analysis}

Our calibration of the models reflects common practice. Table 1 reports the parameter values used for the NK model. For the RBC model we use the same parameter values whenever they are common to both models. We set the discount factor to represent a discount rate of around $4 \%$ per year. Utility function parameters are set consistent with balanced growth. The normalized capital share is set to 0.4 . The investment adjustment cost parameter is set to 2.5 (a common benchmark value, e.g., Christiano, Eichenbaum and Evans (2005)). The price elasticity of demand, $\eta$, ensures a steady-state price mark-up of $20 \%$ over marginal costs. The depreciation rate of capital is $10 \%$ per year. The Calvo parameter implies

\footnotetext{
${ }^{12}$ These results are reported in Appendix D.

${ }^{13}$ Although this variable is redundant when there is zero steady-state inflation (see SchmittGrohé and Uribe (2007)), we introduce it because we also analyzed the results with positive inflation for robustness. The results regarding the reaction of hours did not change and are available on request.
} 
a fixed price duration of 4 quarters. The substitution elasticity is set to a range from 0.4 above Leontief (i.e., at the lower end of the "Chirinko (2008) interval"), at Cobb-Douglas, and at 0.4 above Cobb-Douglas, thus traversing gross complements and gross substitutes. The auto-regressive parameter of technology shocks is set to 0.95 . For simplicity, in our core calibration, we assume monetary policy only responds to deviations of inflation from the steady state with a coefficient just respecting the Taylor principle. ${ }^{14}$ Parameter $v$ is set to equate the real wage expressions in (13) and (15), implying $v=\frac{\left(1-\alpha_{0}\right) r_{0}^{\sigma_{c}}}{\left(r_{0}-\delta \alpha_{0}\right)^{\sigma_{c}}}$. Both models are normalized around the same steady state point.

\begin{tabular}{|c|c|c|}
\hline \multicolumn{3}{|c|}{ Households } \\
\hline \hline$\beta$ & Discount Factor & $1.04^{-1 / 4}$ \\
\hline$\gamma$ & Inverse Frisch Elasticity & 1 \\
\hline$\sigma_{c}$ & Coefficient of Relative Risk Aversion & 1 \\
\hline$v$ & Leisure Scaling Constant & 0.84 \\
\hline \multicolumn{3}{|c|}{ Firms } \\
\hline \hline$\eta$ & Price Elasticity of Demand & 6 \\
\hline$\theta$ & Calvo Parameter & 0.75 \\
\hline$\delta$ & Depreciation Rate & $1.1^{1 / 4}-1$ \\
\hline$\psi$ & Investment Adjustment Costs & 2.5 \\
\hline$\alpha_{0}$ & Normalized Capital Income Share & 0.4 \\
\hline$\sigma$ & Elasticity of Factor Substitution & {$[0.4,1,1.4]$} \\
\hline$\rho$ & Shock Auto-Regressive Parameter & 0.95 \\
\hline \multicolumn{3}{|c|}{ Monetary policy } \\
\hline \hline$\pi$ & Steady State Inflation & 1 \\
\hline$\alpha_{\pi}$ & Taylor Coefficient on Inflation & 1.1 \\
\hline$\alpha_{y}$ & Taylor Coefficient on Output Gap & 0.0 \\
\hline$\alpha_{r}$ & Taylor Coefficient on Lagged Interest Rate & 0.0 \\
\hline
\end{tabular}

Table 1: Parameter Calibration

Figures 1 to 4 depict the dynamic responses of selected variables to a persistent one percentage point increase in $\varepsilon_{t}^{K}$ and $\varepsilon_{t}^{H}$ in the standard RBC and NK models, respectively. ${ }^{15}, 16$ For the NK model, we additionally show the responses of inflation, nominal interest rates, and real marginal costs. The shocks are conducted

\footnotetext{
${ }^{14}$ We relax this in Appendix A, where we analyze the sensitivity to changes in the monetary policy parameters. D also presents results using optimal policy.

${ }^{15}$ We also computed dynamic responses for the Hicks-neutral shock $\varepsilon_{t}^{\mathbb{H}}$ (see Appendix E).

${ }^{16}$ Both models were solved and simulated using first-order approximation methods around their non-stochastic steady state using Dynare, Juillard (2009).
} 
against the parameters values shown in Table 1 with the substitution variations $\sigma \in(0.4,1,1.4)$. Variations in $\sigma$, recall, are admissible in our framework since we expressed the supply side in normalized form.

Summarizing, the effect of the positive technology shocks is to stimulate output, consumption and investment. Movements in factor income shares (excluding Cobb Douglas, where shares are constant) are symmetrical and, following Section 2.2, "favor" either factor depending on the source of the technology improvement and whether factors are gross complements or substitutes.

The figures thus verify that the sign of the hours response on impact depends on $\operatorname{sign}\{\sigma-1\}$. Factor shares and relative prices (marginal productivities) behave as anticipated. Differences in consumption and investment responses corresponding to alternative substitution values are, in turn, explained by, inter alia, investment reactions to changes in relative marginal productivities of capital and labor.

As expected, where qualitative differences may arise lies in the hours response. Furthermore, these differences arise not only across the models but also within them. Whilst capital-augmenting technology-hours impacts appear positive in the standard RBC model, labor-augmenting technology-hours impacts appear negative in the NK one. So far, these results - summarized in the diagonal of Table 2 appear in line with expectations. However, the off-diagonal elements confirm that the models are capable of generating technology-hours impacts of either sign. ${ }^{17}$ For example, for the RBC model, when the substitution elasticity is 0.4 , a negative impact results. For the NK model, the same elasticity induces a positive response.

\begin{tabular}{|l|l|l|}
\hline & RBC & NK \\
\hline$\frac{d H_{t}}{d z_{t}^{K}}$ & $>0$ & $\lesseqgtr 0$ \\
\hline$\frac{d H_{t}}{d z^{H}}$ & $\lesseqgtr 0$ & $<0$ \\
\hline
\end{tabular}

Table 2: Sign Responses

These figures, note, only give a snapshot of our results. They confirm that both models can generate ambiguous responses even for standard parameterizations. In the following section we give a more analytic and generalized interpretation of the technology-hours response in the models. It transpires that the response has threshold characteristics such that technology-hours responses may change sign (a key determinant of which is the value of the substitution elasticity and the source of the technology shock and their interaction with other model features).

To anticipate some of those more general interactions, we perform some additional analysis. Figures $\mathbf{5 a}$ to $\mathbf{5 d}$ analyze the impact response of hours along

\footnotetext{
${ }^{17}$ The negative hours response for the RBC model with labor-augmenting shocks and the positive one for capital-augmenting shocks in the NK model are more pronounced the lower is the value of the substitution elasticity.
} 

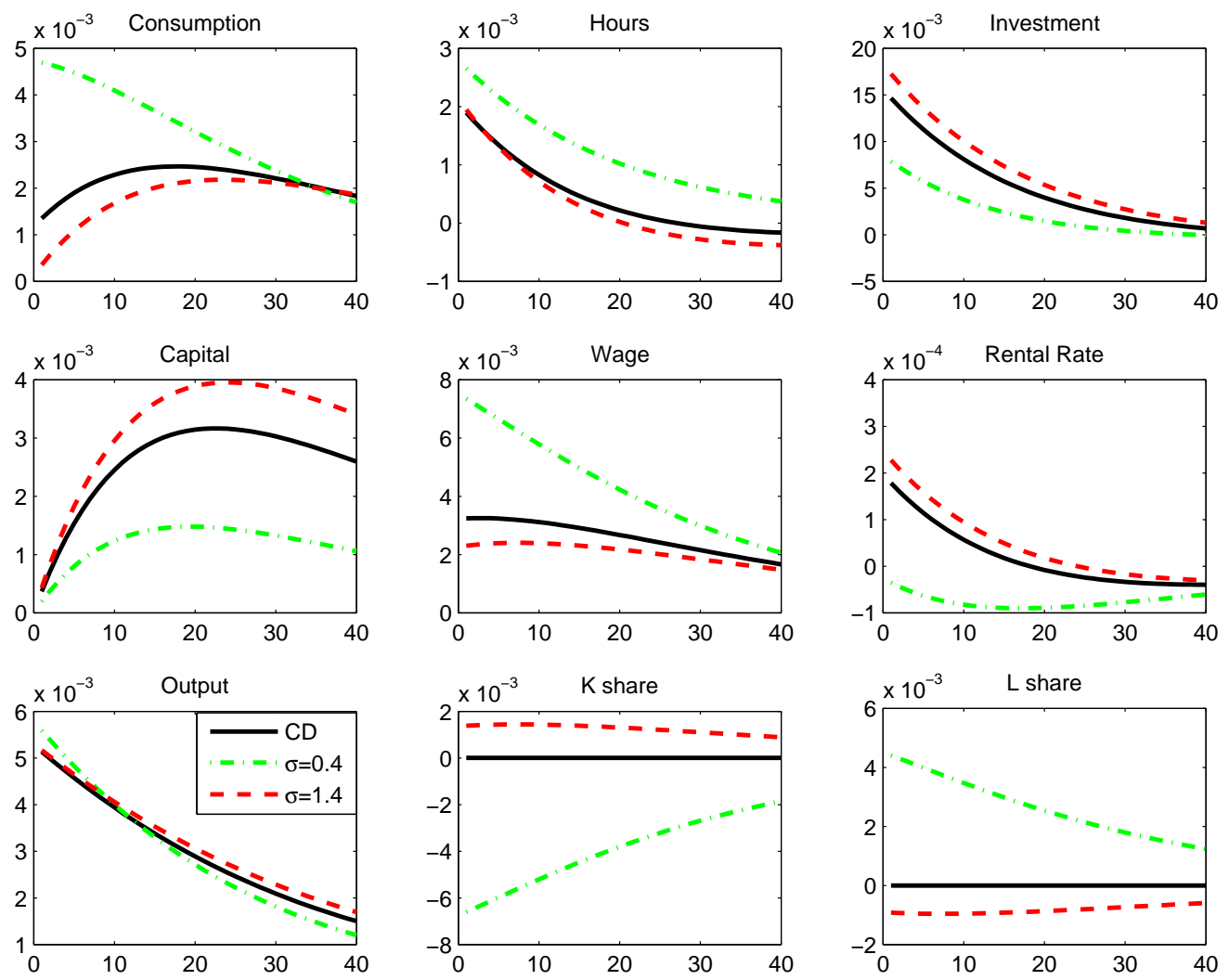

Figure 1: RBC model - Capital Augmenting Shock

a $\sigma \in(0,2]$ support, with a steady-state capital income share of $\alpha_{0}=0.4$ (the baseline) and $\alpha_{0}=0.7$. The latter value is admittedly counter factual, but its use illustrates quite well the threshold characteristics involved. For the RBC model, 5a shows that for all $\sigma$ values sufficiently above $\alpha_{0}$, the labor-augmenting/hours sign flips from negative to positive. For the NK model, it is the capital-augmenting shock that switches sign but as the capital share increases (compare plots (d) to (c)), the threshold point moves inwards.

\section{Technology and Hours: Threshold Rules}

The simulation evidence suggests that for both canonical models the sign response of hours may change for empirically relevant parameter values of the model. We now derive analytical rules determining the sign of the technology-hours impact. 

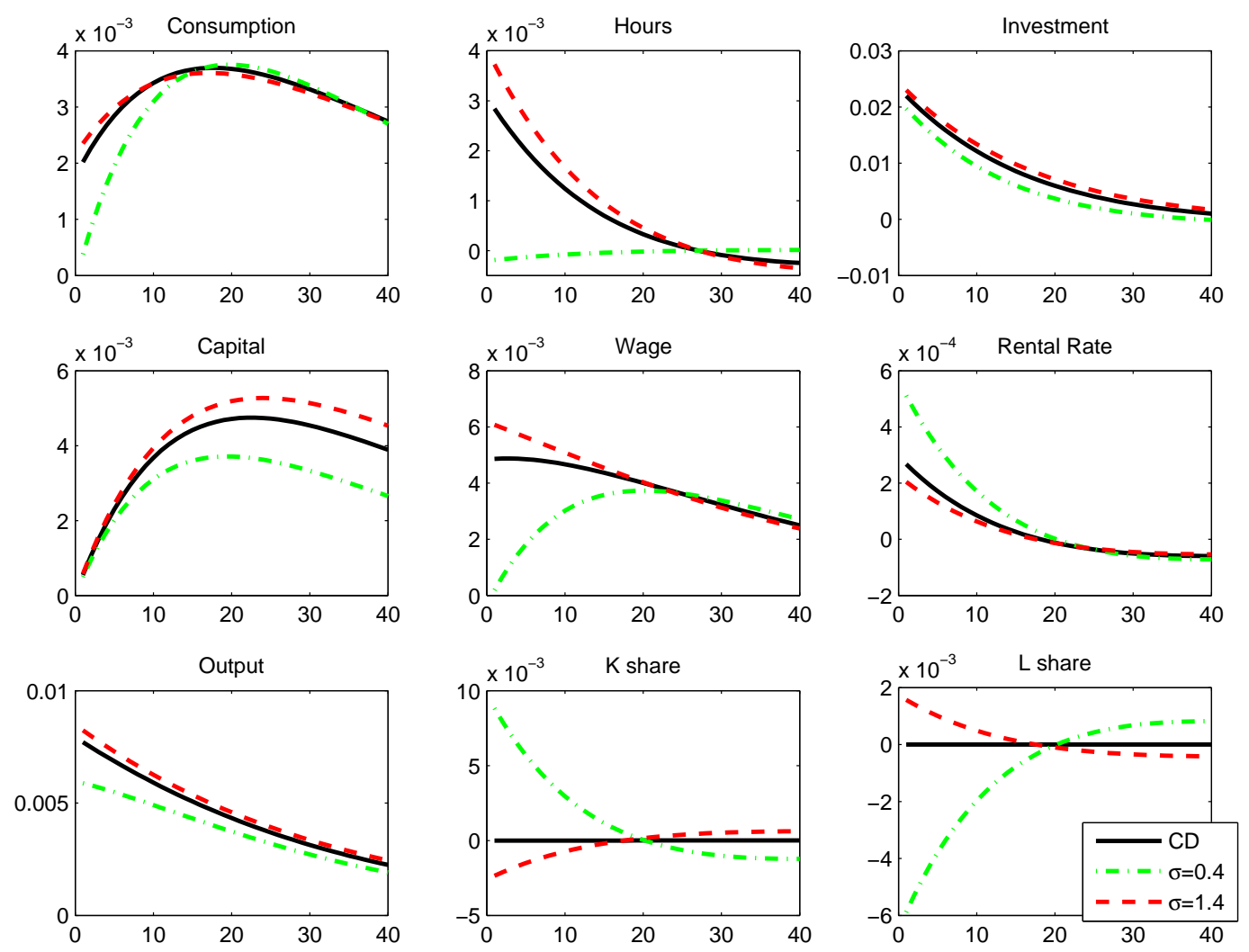

Note: Hours Impact when $\sigma=0.4$ is negative (at -0.00189)

Figure 2: RBC model - Labor Augmenting Shock 

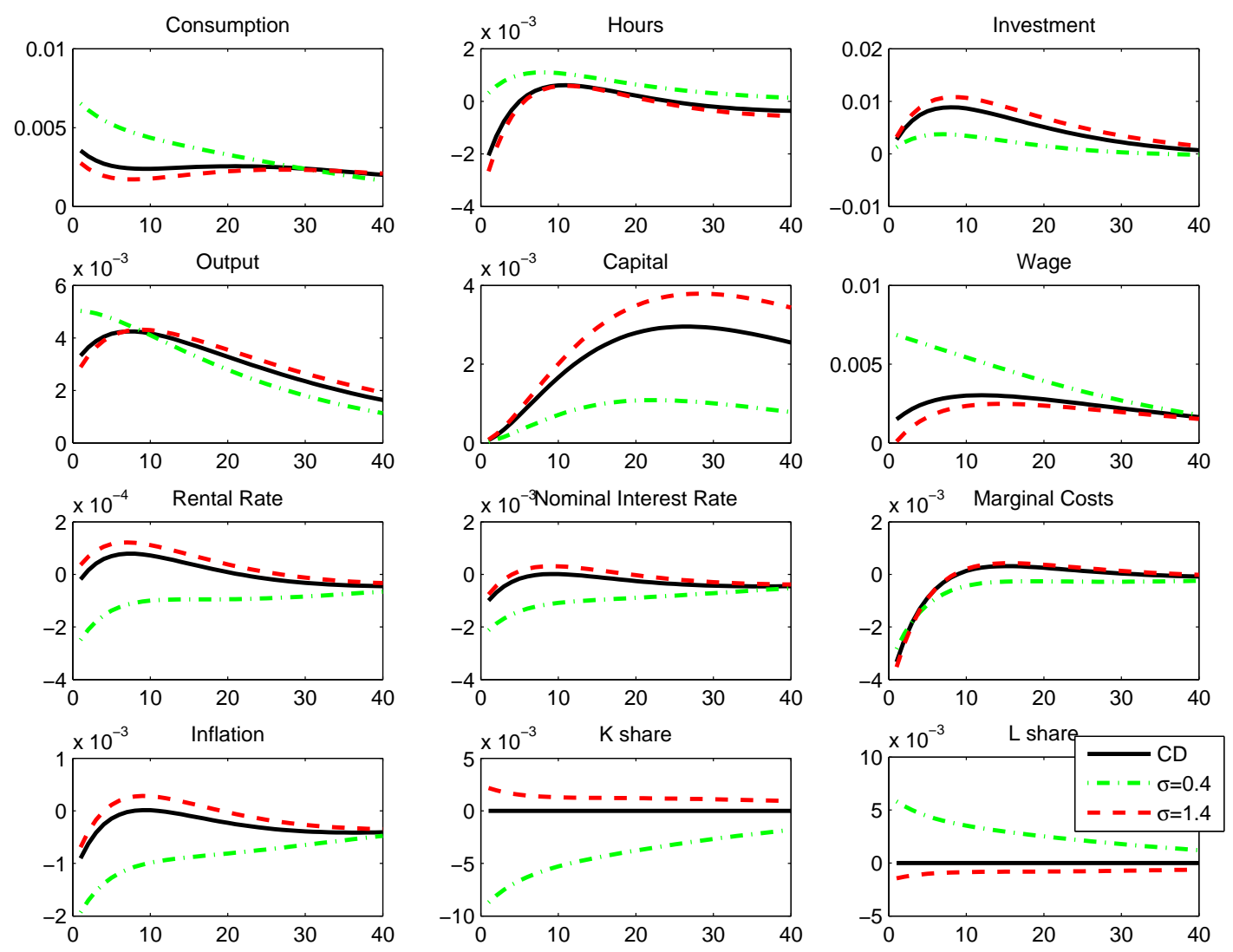

Note: Hours Impact when $\sigma=0.4$ is positive (at 0.0032 )

Figure 3: NK model - Capital Augmenting Shock 

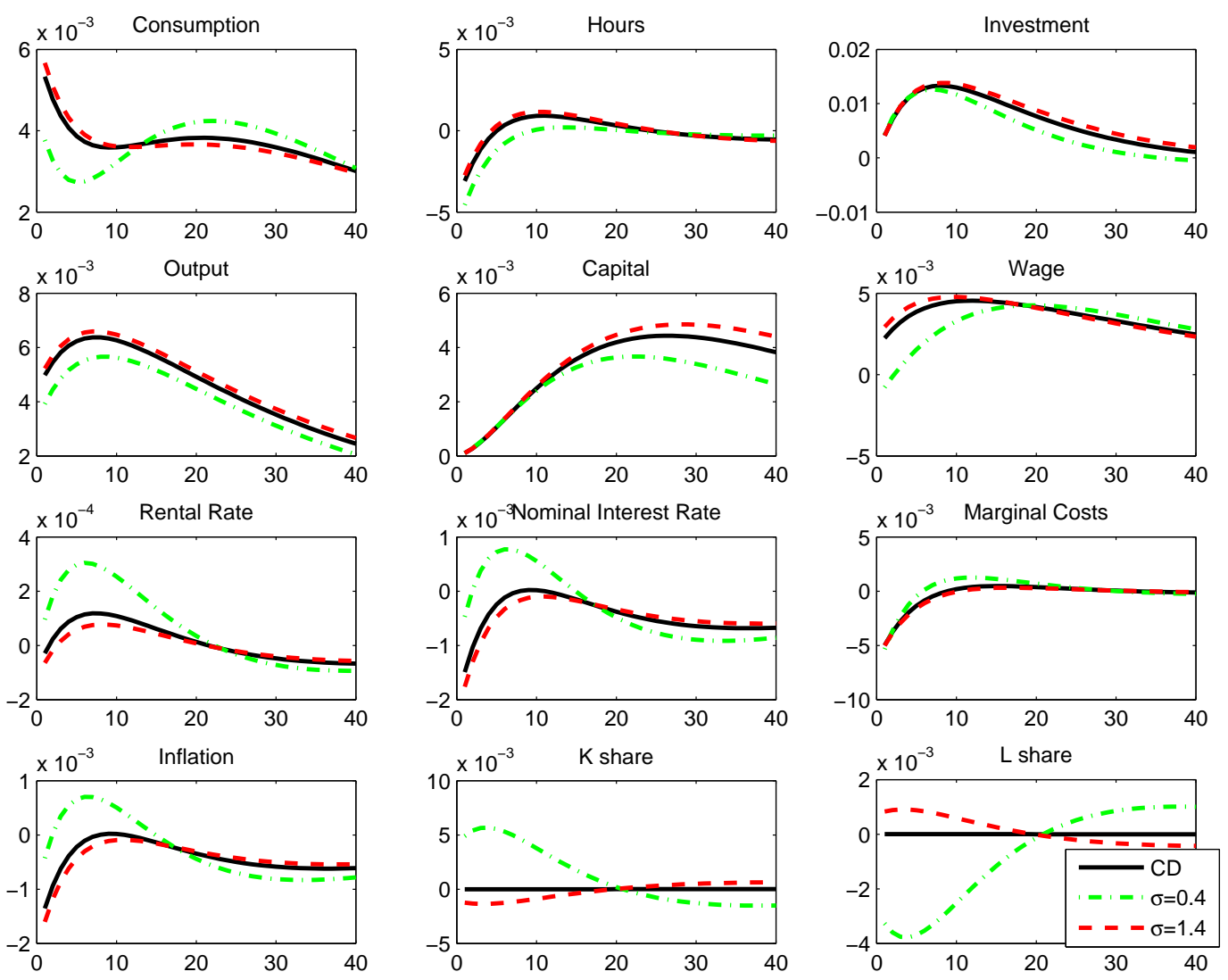

Figure 4: NK model - Labor Augmenting Shock 


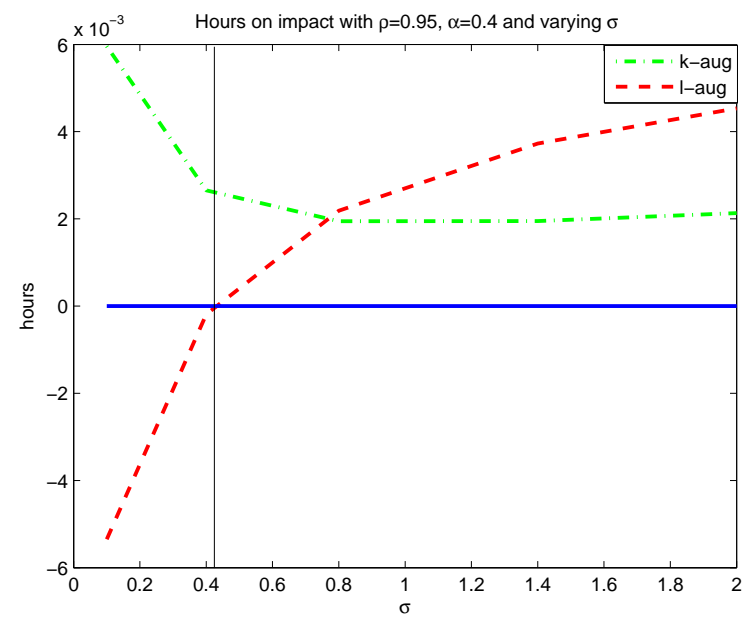

(a) Sensitivity for $\sigma$ with $\alpha=0.4$, RBC Model

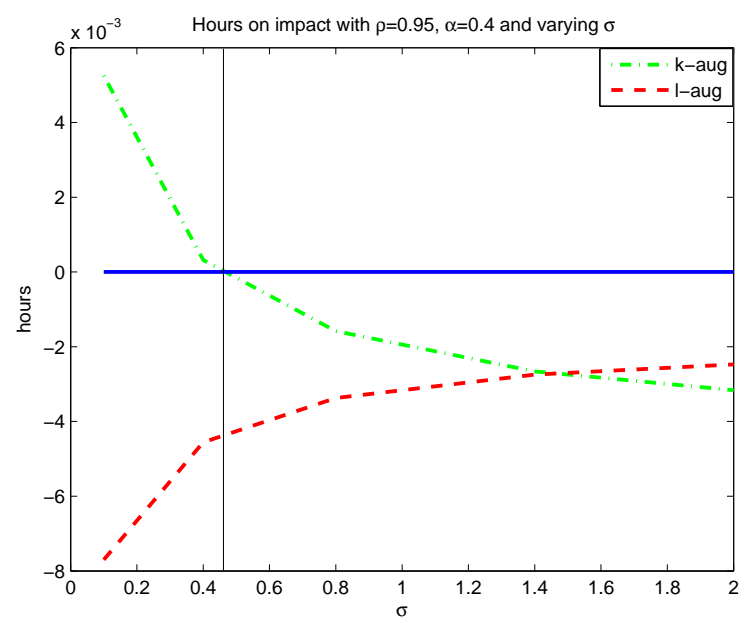

(c) Sensitivity for $\sigma$ with $\alpha=0.4$, NK Model

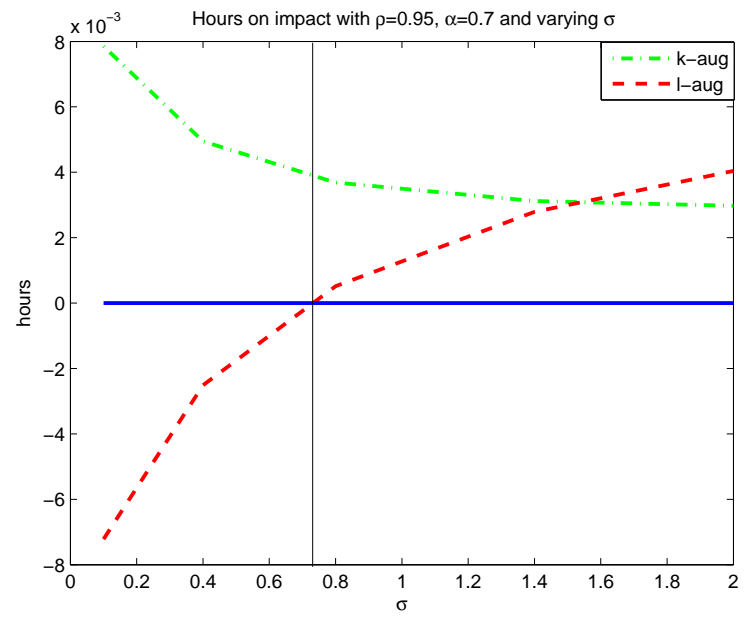

(b) Sensitivity for $\sigma$ with $\alpha=0.7$, RBC Model

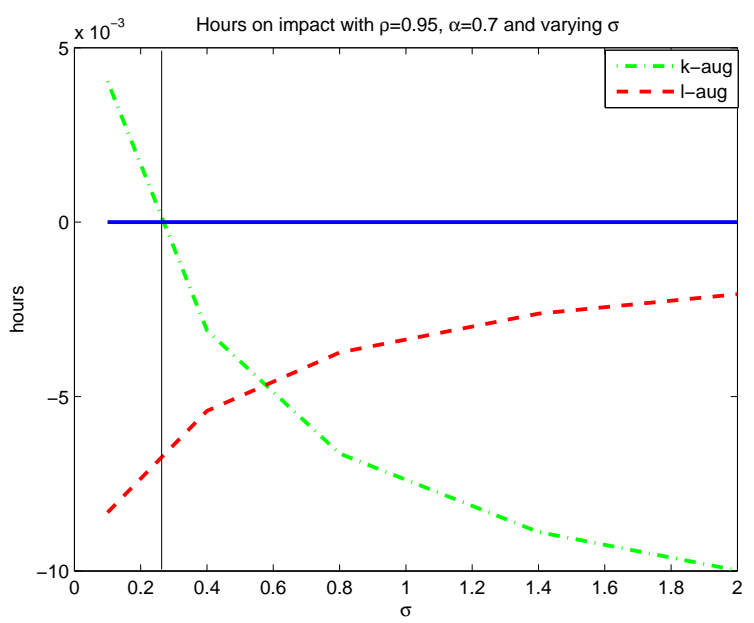

(d) Sensitivity for $\sigma$ with $\alpha=0.7$, NK Model

Figure 5: Changes in $\sigma$ and $\alpha$ 
We first derive the rule for the more general case of the NK model, which nests the $\mathrm{RBC}$ case when prices are flexible and markets are perfectly competitive. The rule reveals some important features usually bypassed by the existing literature. However, to enhance the intuition, we then present the rule for the simpler RBC case under particular assumptions about consumption smoothing. We then frame our discussion (algebraically and graphically) in terms of the effect of technology shocks on labor demand and supply across the two models, and under a variety of parameter configurations.

\subsection{The General Hours-Technology Rule}

Without loss of generality, we normalize supply around the following steady-state: $Y_{0}=H_{0}=1 \Rightarrow K_{0}=\frac{\alpha_{0}}{r_{0}}, w_{0}=1-\alpha_{0}$ and $C_{0}=Y_{0}-\delta K_{0}=\frac{r_{0}-\delta \alpha_{0}}{r_{0}}$. This implies that, around our baseline steady state, $d Y_{t}=d Y_{t} / Y_{0}=d \log Y_{t}$. For simplicity, we abstract from Hicks-neutral shocks $\left(z_{t}^{\mathbb{H}}=0\right) .{ }^{18}$

Consider now the full NK model (of which the standard RBC model is a limiting case). Although the labor supply function is common to both models, a crucial difference is that Calvo price stickiness, $\theta \in(0,1)$, activates the working of the marginal cost channel into NK labor demand.

Zero profits in the steady state imply $\chi=\frac{Y_{0}}{\eta-1}$. Taking this into account, together with normalization, allows us to write production function (34) as:

$$
\begin{gathered}
Y_{t}=\left(\frac{\eta}{\eta-1}\right)\left[\alpha_{0}\left(e^{z_{t}^{K}} \frac{r_{0}}{\alpha_{0}} K_{t-1}\right)^{\frac{\sigma-1}{\sigma}}+\left(1-\alpha_{0}\right)\left(e^{z_{t}^{H}} H_{t}\right)^{\frac{\sigma-1}{\sigma}}\right]^{\frac{\sigma}{\sigma-1}}-\frac{1}{\eta-1} \quad(49) \\
\Rightarrow d \log Y_{t}=\left(\frac{\eta}{\eta-1}\right) d \log C E S_{t}=\left(\frac{\eta}{\eta-1}\right)\left[\alpha_{0} d z_{t}^{K}+\left(1-\alpha_{0}\right)\left(d z_{t}^{H}+d \log H_{t}\right)\right]
\end{gathered}
$$

To proceed, note that the rhs of (15) is the normalized CES condition for $F_{H}$ and, hence, on the basis of the NK marginal cost equation (38), the FOC for labor, we can write,

$$
d \log w_{t}=\frac{\sigma-1}{\sigma} d z_{t}^{H}+\frac{1}{\sigma} d \log C E S_{t}-\frac{1}{\sigma} d \log H_{t}+d \log m c_{t}
$$

Now (50) and (51) imply the following labor demand relation:

$$
d \log w_{t}=d \log m c_{t}+\frac{\alpha_{0}}{\sigma} d z_{t}^{K}+\frac{\sigma-\alpha_{0}}{\sigma} d z_{t}^{H}-\frac{\alpha_{0}}{\sigma} d \log H_{t} .
$$

\footnotetext{
${ }^{18}$ These are examined in Appendix E.
} 
Equations (28) and (30), in turn, imply the labor supply relation:

$$
d \log w_{t}=\gamma d \log H_{t}+\sigma_{c} d \log C_{t} .
$$

The equilibrium change in hours, thus, can be found by combining (52) and (53). After some transformations, this yields:

$$
d \log H_{t}=\frac{1}{\Upsilon}\left[d \log m c_{t}+\frac{\alpha_{0}}{\sigma} d z_{t}^{K}+\frac{\sigma-\alpha_{0}}{\sigma} d z_{t}^{H}-\sigma_{c} d \log C_{t}\right],
$$

where $\Upsilon=\frac{\gamma \sigma+\alpha_{0}}{\sigma}$.

The impact effect of technology shocks on equilibrium hours is then given by:

$$
\begin{gathered}
\frac{d \log H_{t}}{d z_{t}^{H}}=\frac{1}{\Upsilon}\left[\frac{d \log m c_{t}}{d z_{t}^{H}}+\frac{\sigma-\alpha_{0}}{\sigma}-\sigma_{c} \frac{d \log C_{t}}{d z_{t}^{H}}\right] . \\
\frac{d \log H_{t}}{d z_{t}^{K}}=\frac{1}{\Upsilon}\left[\frac{d \log m c_{t}}{d z_{t}^{K}}+\frac{\alpha_{0}}{\sigma}-\sigma_{c} \frac{d \log C_{t}}{d z_{t}^{K}}\right],
\end{gathered}
$$

Given that $\Upsilon>0$, the sign of the technology-hours correlation depends on the signs of the elements in brackets in (55) and (56). The first two elements in both expressions reflect the impact of shocks through labor demand shifts: changes in the marginal cost and changes in the marginal product of labor (through both increased output and factor substitution). The third element reflects the impact of shocks through changes in labor supply decisions. Recall that labor supply increases only if wages change faster than consumption (weighted by the coefficient of relative risk aversion).

Given the potential relevance of the reaction of consumption, we can develop further (55) and (56) so as to enhance the intuition. For a given shock $d z_{t}^{i}(i=$ $H, K)$, around the baseline steady state, we know that:

$$
\frac{d \log C_{t}}{d z_{t}^{i}}=\frac{d \log C_{t}}{d \log Y_{t}} \cdot \frac{d \log Y_{t}}{d z_{t}^{i}}=\frac{d C_{t}}{d Y_{t}} \frac{Y_{0}}{C_{0}} \cdot \frac{d \log Y_{t}}{d z_{t}^{i}}=\frac{m p c_{t}}{a p c} \cdot \frac{d \log Y_{t}}{d z_{t}^{i}}
$$

where $m p c_{t}$ is the marginal propensity to consume, and apc is the average propensity to consume in the baseline steady state period $\left(C_{0} / Y_{0}>0\right)$. We can then substitute $\frac{d \log Y_{t}}{d z_{t}^{i}}$ in this expression by making use of (50). The resulting expression can then be used in conjunction with (55) and (56). This yields the expressions for the impact of both shocks as functions of the parameters of the model, $m p c_{t}$, and the reaction of the marginal cost: 


$$
\begin{gathered}
\frac{d \log H_{t}}{d z_{t}^{H}}=\frac{1}{\Upsilon+\sigma_{c} \frac{m p c_{t}}{a p c} \phi\left(1-\alpha_{0}\right)}\left[\frac{d \log m c_{t}}{d z_{t}^{H}}+\frac{\sigma-\alpha_{0}}{\sigma}-\sigma_{c} \frac{m p c_{t}}{a p c} \phi\left(1-\alpha_{0}\right)\right] \\
\frac{d \log H_{t}}{d z_{t}^{K}}=\frac{1}{\Upsilon+\sigma_{c} \frac{m p c_{t}}{a p c} \phi\left(1-\alpha_{0}\right)}\left[\frac{d \log m c_{t}}{d z_{t}^{K}}+\frac{\alpha_{0}}{\sigma}-\sigma_{c} \frac{m p c_{t}}{a p c} \phi \alpha_{0}\right]
\end{gathered}
$$

where $\phi=\frac{\eta}{\eta-1}>1$.

Now we are in a position to establish the threshold values for $\sigma$ that would imply a change in the sign of the response in hours, our general threshold rules: ${ }^{19}$

$$
\begin{array}{llc}
\frac{d \log H_{t}}{d z_{t}^{H}}>0 \quad \text { if } & \sigma>\frac{\alpha_{0}}{1+\frac{d \log m c_{t}}{d z_{t}^{H}}-\sigma_{c} \frac{m p c_{t}}{a p c} \phi\left(1-\alpha_{0}\right)} \\
\frac{d \log H_{t}}{d z_{t}^{K}}>0 & \text { if } & \sigma<\frac{\alpha_{0}}{\sigma_{c} \frac{m p c_{t}}{a p c} \phi \alpha_{0}-\frac{d \log m c_{t}}{d z_{t}^{K}}} .
\end{array}
$$

The key margins for the rules are the $\sigma-\alpha_{0}$ wedge, the capital income share, risk aversion and the response of the marginal propensity to consume. The latter two, though, are linked since the higher is $\sigma_{c}$ (equivalently, the lower is the elasticity of inter-temporal substitution), the less able are agents to translate income gains into a smooth consumption profile and, thus, ceteris paribus, the higher is the impact of the marginal propensity to consume. The latter, as we shall discuss below, is also strongly related to the persistence of shocks as well as to the strength of investment adjustment costs.

Both expressions (60) and (61) highlight the fact that the responses of hours can take any sign depending on the kind of shock hitting the economy and the deep parameters of the model. The threshold, however, appears to be a complicated expression. We will make more sense of it in the next two sections when presenting simplified rules for the RBC model and a graphical interpretation.

\subsection{The Simple Hours-Technology Rule}

Consider the standard RBC model (no investment adjustment costs, perfect competition, no price stickiness). Thus, $\phi \rightarrow 1$ and $d \log m c_{t}=0$. The simplified threshold rules for the substitution elasticity then become:

\footnotetext{
${ }^{19}$ We assume that the expression outside the brackets is positive. Since $\Upsilon$ is positive, then this will be the case so long as $m p c_{t}$ is positive (or a small negative number). Also, the rule for labor-augmenting shocks holds if $\left[1+\frac{d \log m c_{t}}{d z_{t}^{H}}-\sigma_{c} \frac{m p c_{t}}{a p c} \phi\left(1-\alpha_{0}\right)\right]>0$ which is almost sure to be the case.
} 


$$
\begin{array}{lll}
\frac{d \log H_{t}}{d z_{t}^{H}}>0 & \text { if } & \sigma>\frac{\alpha_{0}}{1-\sigma_{c} \frac{m p c_{t}}{a p c}\left(1-\alpha_{0}\right)} \\
\frac{d \log H_{t}}{d z_{t}^{K}}>0 & \text { if } & \sigma<\frac{1}{\sigma_{c} \frac{m p c_{t}}{a p c}}
\end{array}
$$

Accordingly, we can now rationalize the responses of hours found in our baseline calibration and summarized in Table 2 for the RBC model. If the consumption smoothing motive is strong, which will depend on the persistence of shocks in the RBC model (and also the investment adjustment costs in the NK model), then $m p c_{t}$ will be small. This implies that $\frac{d \log H_{t}}{d z_{t}^{K}}>0$ for almost all empirically relevant values of $\sigma$.

For $\frac{d \log H_{t}}{d z_{t}^{H}}$ we have a positive response for $\sigma \varpi>\alpha_{0}$ where $\varpi=1-\sigma_{c} \frac{m p c_{t}}{a p c}(1-$ $\alpha_{0}$ ). If the consumption smoothing motive was such that $m p c_{t} \rightarrow 0$, then $\varpi \rightarrow 1$, and the threshold value for $\sigma$ would simply be $\alpha_{0}$. This case would imply that the shock has a negligible effect on labor supply and all changes in hours are driven by changes in labor demand. For the labor-augmenting case, the shock has a factor substitution effect on labor demand equal to $\frac{\sigma-1}{\sigma}$, and an output effect equal to $\frac{1-\alpha_{0}}{\sigma}$. The sign of the sum of these effects on labor demand will then depend on $\operatorname{sign}\left[\sigma-\alpha_{0}\right]^{20}$

For the NK case, in turn, we need to consider the effects of shocks on the real marginal cost. For the parameter values used (and for most parameterizations), the real marginal cost falls after a technology shock. Looking at expressions (60) and (61), it is easy to see that we can now find a switching sign for the capitalaugmenting shock. For the labor-augmenting shock, sign changes may still happen, but it is most likely that $\sigma$ will be below the threshold.

\section{Hours and Technology:}

\section{A Labor Demand and Supply Interpretation}

Consider again the labor demand and supply schedules arising under flexible prices. From (52) and (53), considering the flexible prices RBC case, we obtain:

$$
d \log H_{t}^{D}=-\frac{\sigma}{\alpha_{0}} d \log w_{t}+\left(\frac{\sigma-\alpha_{0}}{\alpha_{0}}\right) d z_{t}^{H}+d z_{t}^{K}
$$

\footnotetext{
${ }^{20}$ In other words, as discussed below, this arises if there is complementarity between labor and labor-augmenting technical change. However, this property is not sufficient to generate positive hours changes to labor-augmenting shocks as should be clear from (62) and (63).
} 


$$
d \log H_{t}^{S}=\frac{1}{\gamma+\sigma_{c} \frac{m p c t}{a p c}\left(1-\alpha_{0}\right)}\left\{d \log w_{t}-\sigma_{c} \frac{m p c_{t}}{a p c}\left[\left(1-\alpha_{0}\right) d z_{t}^{H}+\alpha_{0} d z_{t}^{K}\right]\right\}
$$

Straightforwardly, these two schedules can be graphed in the hours-wage space and the resulting shifts from changes in shocks and parameters analyzed accordingly. This is done in Section 7.3. Before then however, we analyze each schedule in turn.

\subsection{Technology Shocks and Hours Demand}

Whilst the effect of capital-augmenting shocks on labor demand is independent of factor substitution, whether positive labor-augmenting shocks increase labor demand depends on whether the substitution elasticity exceeds the capital income share.

As mentioned, this property arises if there is complementarity between labor demand and labor-augmenting technical progress, $\frac{\partial^{2} Y}{\partial \Gamma^{H} \partial H}>0$ and is straightforward to show. ${ }^{21}$ Complementarity, though, is neither necessary nor sufficient to produce a positive hours response following $d z_{t}^{H}>0$ since the net effect depends on labor supply reactions and - in the NK case - the effect of the reaction of real marginal costs on labor demand.

For the familiar cases of Cobb-Douglas (respectively, Leontief) this effect is always positive (negative). Note, though, that a labor-augmenting technology shock will decrease labor demand within the interval $\sigma \in\left[0, \alpha_{0}\right)$.

In the Leontief case, labor demand is unresponsive to real wages (as expected under a fixed-proportions production) and falls one-for-one with positive labor augmenting technical improvements. Only positive capital-augmenting shocks raise labor demand (one-for-one).

\subsection{Technology Shocks and Hours Supply}

Looking at equation (65), shifts in labor supply depend on the Frisch-elasticity and risk aversion. They also depend on the endogenous responses of marginal consumption decisions. In the conventional $m p c_{t}>0$ case, labor supply is positively

\footnotetext{
${ }^{21}$ Given production function (1), if labor-augmenting technical progress raises labor demand we would have,

$$
\frac{\partial^{2} Y}{\partial \Gamma^{H} \partial H}=f(\kappa)-\kappa f^{\prime}(\kappa)+\kappa^{2} f^{\prime \prime}(\kappa)>0
$$

Exploiting the definition of the substitution elasticity (equation (2)) and noting that $\alpha=\frac{\kappa f^{\prime}(\kappa)}{f(\kappa)}$, it follows that $\sigma-\alpha=\frac{f^{\prime}(\kappa)}{f(\kappa)}\left[f(\kappa)-\kappa f^{\prime}(\kappa)+\kappa^{2} f^{\prime \prime}(\kappa)\right]$, which when abstracting from $\frac{f^{\prime}(\kappa)}{f(\kappa)}>$ 0 retrieves $(\mathrm{f} 1)$.
} 
related to the real wage but is decreasing in both technical progress terms (the latter reflects the familiar dominance of the income effect over the substitution effect). ${ }^{22}$

For the most part, the strength of the mpc responses depend on the values of investment adjustment costs and risk aversion and also on how persistent the technology shocks are. ${ }^{23}$ As equation (26) shows, investment adjustment costs inhibit capital accumulation and hence future consumption possibilities. Such costs raise the trade-off between current and future consumption in favor of the former. In the limit of infinite costs, the investment/saving motive disappears, leaving all extra income to be consumed, $m p c \rightarrow 1$.

However, even without such costs, consumption smoothing works to decrease the initial fraction of income consumed. When technology shocks are temporary, $m p c_{t}$ is positive and small with all feasible parameters. ${ }^{24}$ Euler equation (12) reminds us that current consumption depends positively on future consumption and negatively on future capital productivity. Via (16), current output and the marginal product of capital increase inducing a positive capital-stock effect. If the shock is temporary, most of the positive output effect rapidly disappears while the increased capital stock remains persistently at a higher level. Hence, nextperiod marginal product of capital decreases marginally below the steady state. Because of the increasing next-period consumption and the decreasing next-period marginal product of capital, current consumption rises.

\subsection{A Graphical Analysis}

For simplicity, we use the RBC case for a graphical analysis. With $\log H_{t}$ on the vertical axis and $\log w_{t}$ on the horizontal, the labor demand schedule (64) is always downward sloping with the slope converging towards the horizontal (i.e., flattening) as $\sigma \rightarrow 0$. With $\gamma>0$ and, at least, $m p c_{t} \geq 0$, the labor supply schedule is upward sloping. ${ }^{25}$

\footnotetext{
${ }^{22}$ In the context of a permanent (or highly persistent) productivity shock the consumption effect is ambiguous: future marginal product of capital increases with a negative impact on current period consumption. The higher is the intertemporal substitution (i.e. the inverse of risk aversion $\sigma_{c}$ ) the stronger is that negative effect raising the possibility that $m p c_{t}<0$ (e.g., agents deny themselves current consumption to accelerate the transition to a higher level of capital and income).

${ }^{23}$ Accordingly, it is worth stressing that even though our emphasis is on the initial hours response, this response is nonetheless determined by the entire dynamic trajectory of the model, its forward-lookingness and shock persistence.

${ }^{24}$ The following is based on extensive simulation exercises. Tables analyzing a wide range of cases are provided in Appendix C.

${ }^{25} \mathrm{~A}$ negative slope is also possible but that would require that $m p c_{t}<-\gamma \frac{a p c}{\sigma_{c}\left(1-\alpha_{0}\right)}$ which appears unlikely.
} 


\subsubsection{The Labor Augmenting Shock}

As regards a labor augmenting shock the relative size of the substitution elasticity and the capital income share is crucial in two ways. If $\sigma>(<) \alpha_{0}$ (see Figures $\mathbf{6 a}$ and $\mathbf{6 b}$ ), then (i) the negative slope exceeds (is below) unity, in absolute value, and (ii) the shift of the demand schedule is upwards (downwards) in response to a positive labor augmenting shock.

Regarding supply reactions, as already discussed, in the context of a temporary shock, the marginal propensity to consume is always small and positive implying an upward-sloping labor supply (Figure 7a). However, when coupled with a permanent (or highly persistent) shock and/or low risk aversion the mpc-reaction is more likely to be negative. This steepens labor supply compared to Figure 7a and a positive labor-augmenting shock shifts labor supply upwards (Figure 7b).

Accordingly, we conclude there are two unambiguous sign cases. Combining the cases of Figures $\mathbf{6 a}$ and $\mathbf{7 b}$ - i.e. both demand and supply shift up - the hours-technology impact is positive. This is so when $\sigma \geq \alpha_{0}$ and $m p c_{t}<0$. Combining Figures $\mathbf{6 b}$ and $7 \mathbf{a}$ cases - i.e., both curves shift downwards - the hours-technology impact is negative. This is so when $\sigma<\alpha_{0}$ and $m p c_{t}>0$.

In turn, combining the cases described by Figures $\mathbf{6 a}$ and $7 \mathbf{a}$, on one hand, and $6 \mathrm{~b}$ and $7 \mathrm{~b}$, on the other, results in an ambiguous hours sign. In both cases, the sign depends, besides the relative sizes of the shifts, also on the relative slopes of the demand and supply curves. Empirically, the former case, i.e. the combination of Figure $\mathbf{6 a}$ and $\mathbf{7 a}$, appears by far the more relevant. In that case, it is clear that the more $\sigma$ exceeds $\alpha_{0}$ the more probable it is that the positive upward shift of labor demand dominates the downward shift of labor supply.

However, $\sigma \rightarrow \infty$ does not guarantee a positive technology-hours impact. On the demand side, while the widening of the $\sigma-\alpha_{0}$ wedge increases the upward shift of demand, the downward slope of the demand curve steepens as $\sigma$ rises and compensates, at least partly, the rightward shift in the intersection point of the demand and the supply curves.

The supply-curve, in turn, becomes flatter and its downward shift larger the higher the $m p c_{t}$, on one hand, and the higher the risk aversion, on the other. We know that the rising adjustment costs raise $m p c_{t}$ towards unity. This flattens the upward slope of the supply curve and increases the size of its downward shift. Hence, with sufficiently high $m p c_{t}$ and risk aversion the downward shift of the supply curve dominates the upward shift of demand even if the latter shift approaches infinity. This case is depicted by Figure 8 .

\subsubsection{The Capital Augmenting Shock}

Positive capital augmenting shocks shift the demand curve always upwards. Independently from the size of substitution elasticity or other model parameters, the 

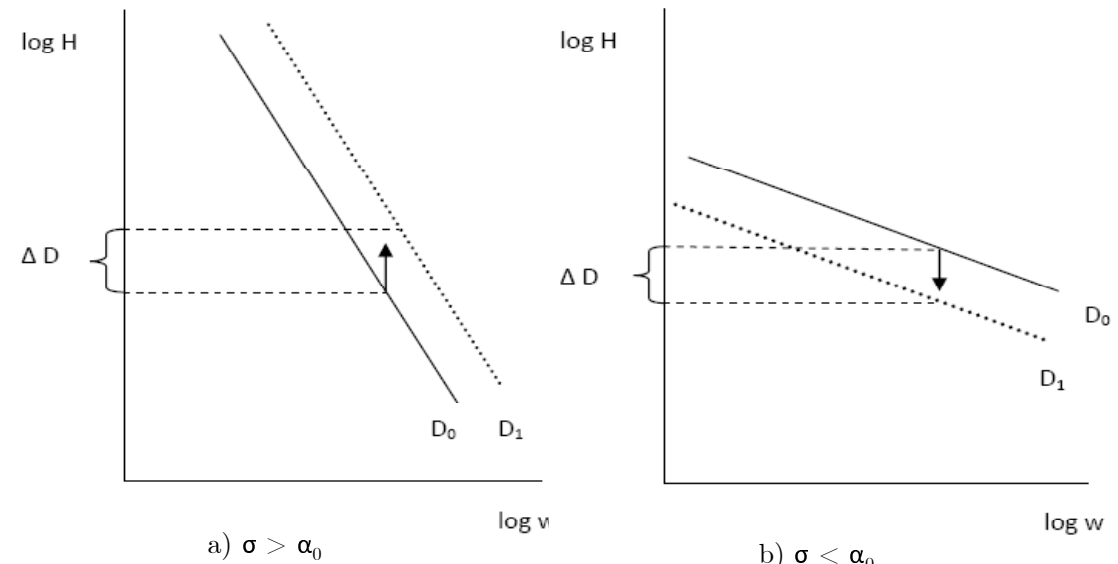

b) $\sigma<\alpha$

Figure 6: Changes in labor demand.

semi-elasticity of the labor demand with respect to the capital augmenting shock is unity.

Hence, the hours impact from the capital-augmenting shock is unambiguously positive only if the $m p c$-impact is negative and both the demand and supply curves shift upwards. As discussed earlier, this requires that the shock is highly persistent and risk aversion not too high. In all other cases, labor demand shifts upward and labor supply downwards and the sign of the shift of the intersection point is ambiguous.

However, we know that the downward slope of the demand curve steepens when $\sigma$ increases and that the upward shift of the demand curve remains the same as a response to a capital augmenting shock. Hence, when the downward shift of the supply curve is coupled with the upward shift of two alternative demand curves, the equilibrium related to the steeper demand curve is to the left and downside of the intersection point corresponding to the less steeply downwards sloping demand curve. These cases are depicted in Figure $\mathbf{9 a}$ and $\mathbf{9 b}$ where the initial equilibrium point is at point $A$ and the two alternative equilibria after the shock are at point $B$ for high (panel a) and low (panel b) values of $\sigma$.

This implies that when substitution elasticity is low (i.e. labor demand is flat) the possibility that the hours-technology impact of the capital augmenting shock is positive increases compared to the case when substitution elasticity is higher (i.e. demand is steep). 


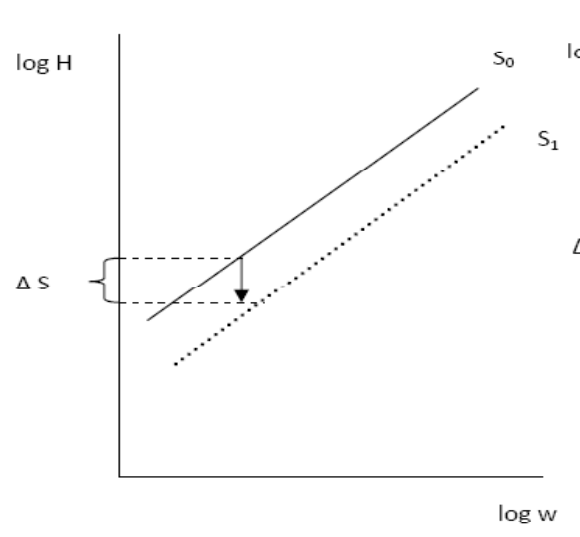

a) $\mathrm{mpc}_{\mathrm{t}}>0$

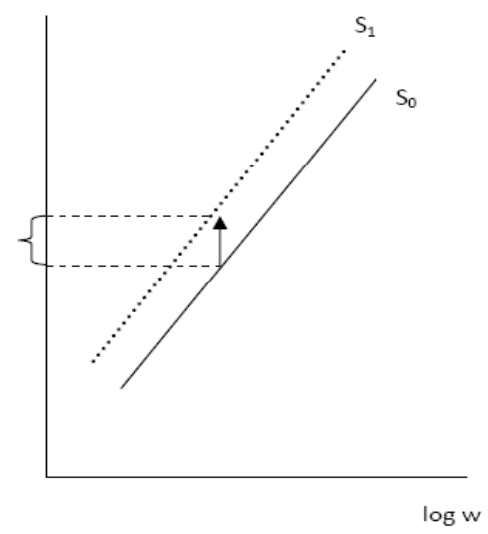

b) $\mathrm{mpc}_{\mathrm{t}}<0$

Figure 7: Changes in labor supply.

\subsection{Labor Demand and Supply in the NK Model}

The impact effects of technology shocks on labor demand and supply are defined by equations (52) and (53), the latter common to both models. Price stickiness, however, implies that real marginal costs deviate from their steady-state level and affect labor demand. Interestingly the marginal cost channel affects the shift of the demand curve but not its slope. The crucial questions are

(i) whether marginal cost reactions strengthen or compensate the shift effects that could otherwise occur without price stickiness, and;

(ii) how big these effects are.

There is no clear-cut answer to the first issue and, in fact, as our numerical analysis (Appendix C) indicates, real marginal costs may, depending on the values of model parameters, either increase or decrease as a response to technology shocks. The answer to the second issue is more clear-cut because the importance of the marginal cost channel is by definition positively related to Calvo-parameter $\theta$. Thus with small $\theta$, the marginal cost channel, independently from the sign of the effect, has only a minor effect on the shift of the demand curve and therefore our previous analysis remain qualitatively essentially unchanged. The importance of the marginal cost channel strengthens as $\theta \rightarrow 1$. However, to validate even approximately the conventional wisdom that the hours impact of labor augmenting shock is negative, would require that only a small deviation of $\theta$ from zero suffices to render the marginal cost channel dominating and that with a very wide range of 


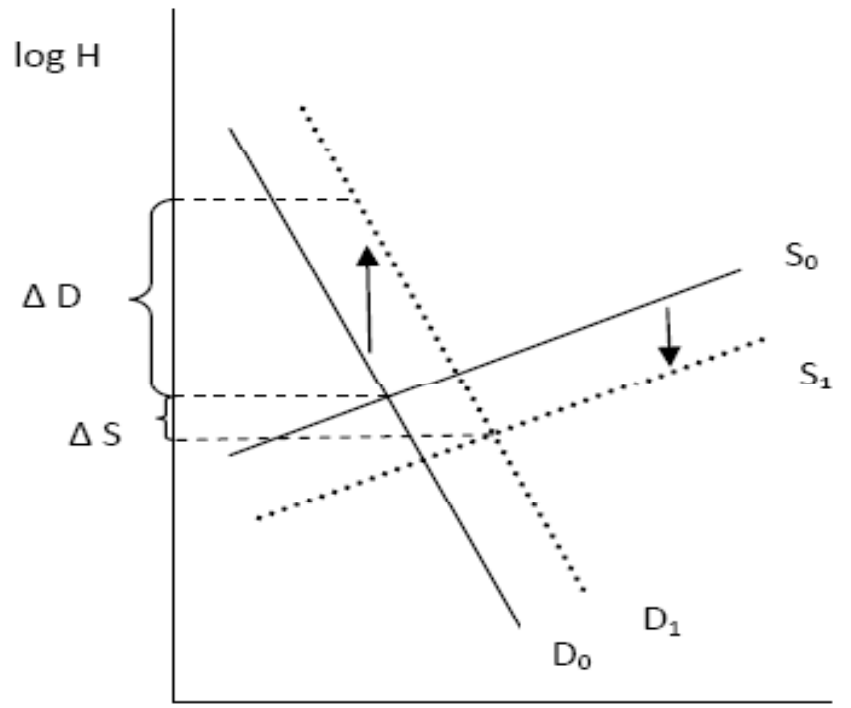

$\log w$

Figure 8: Labor augmenting shock: $\sigma>\alpha_{0}$ and $m p c_{t}>0$.

feasible parameter values of the model the effect of this channel on labor demand is negative. 


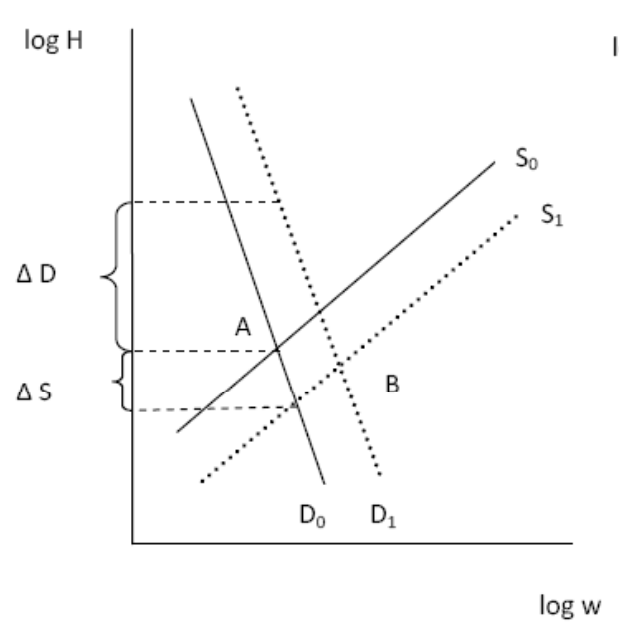

a) $\operatorname{High} \sigma$

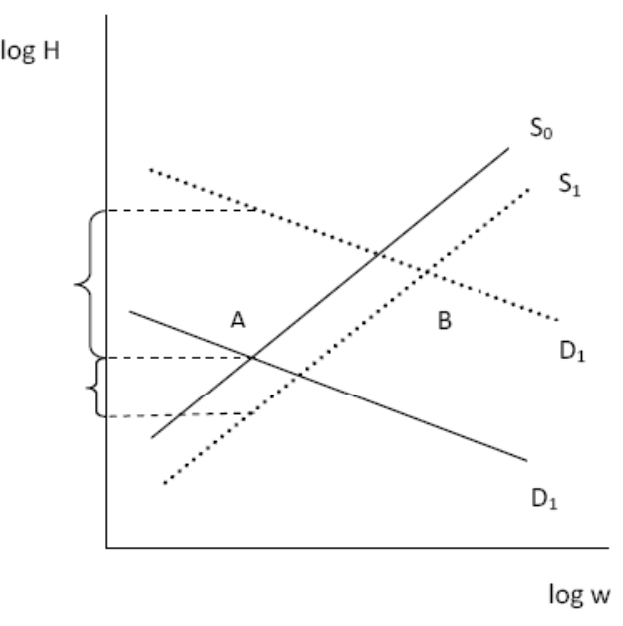

b) Low $\sigma$

Figure 9: Capital augmenting shock.

To provide further evidence regarding this point, Figure $\mathbf{1 0}$ plots the impact effect of both technology shocks in the NK model using the baseline values of the parameters in Table 1 but changing simultaneously the Calvo parameter $(\theta)$ and the investment adjustment cost parameter $(\psi)$. Parameter $\psi$ is important as it affects the marginal propensity to consume. One result worth mentioning is that, for capital-augmenting shocks with low values of $\sigma$, the response remains positive even for high values of $\theta$ and for all values of $\psi$ considered. It is also noticeable that when investment adjustment costs are low, very high levels of price rigidity generate a positive rather than negative hours effect for high $\sigma$ values.

\section{Some Robustness analysis}

The appendices provide a wide range of robustness exercises. For reasons of space, we do not devote much space commenting them here. Appendix A reports the impact response of hours varying several key parameters of the model. Appendix $B$ derives the labor supply equation and the threshold rules for non-separable utility. This an important case as it has been used widely in the literature given its compatibility with no secular trend in the share of hours allocated to work. Appendix D presents the results from a model in which monetary policy is optimal, and not derived from a simple non-optimized Taylor-type rule. The results show 


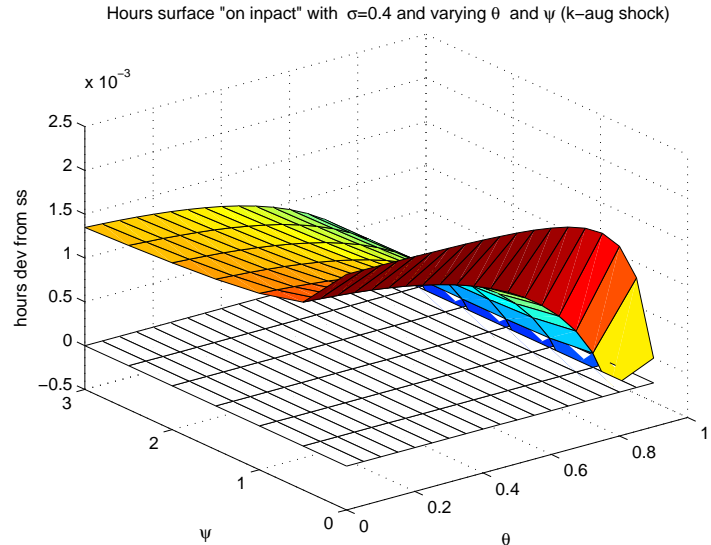

(a) K-aug $\sigma=0.4$

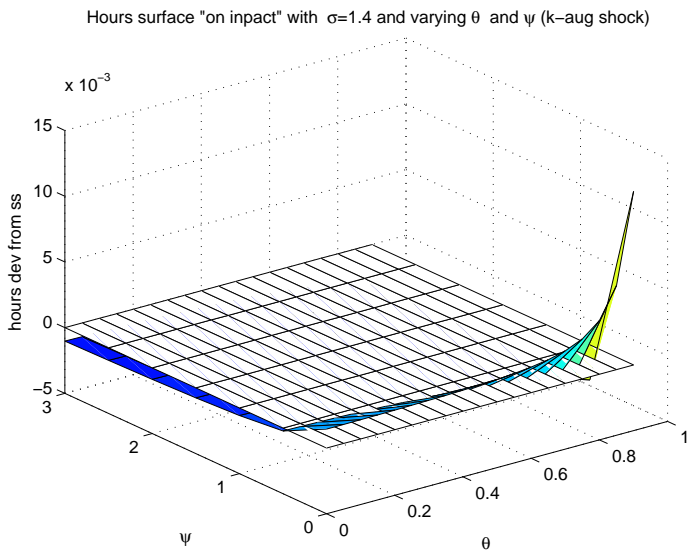

(c) K-aug $\sigma=1.4$

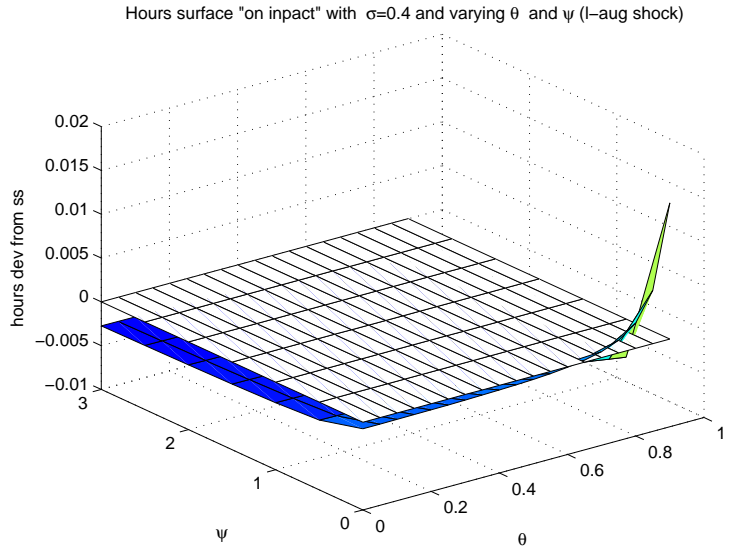

(b) L-aug $\sigma=0.4$

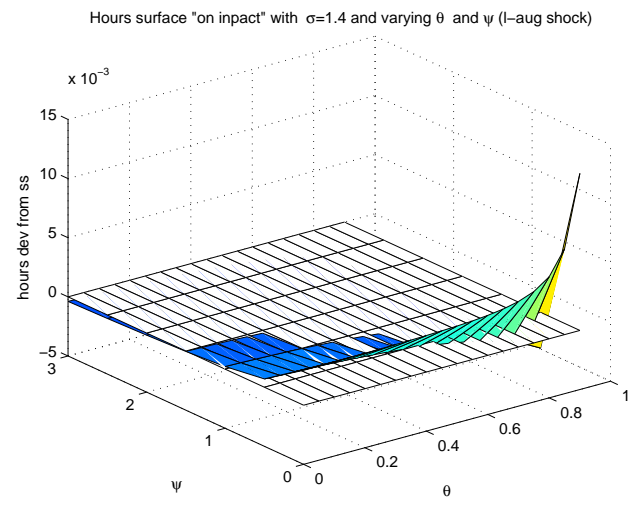

(d) L-aug $\sigma=1.4$

Figure 10: Sensitivity analysis in the NK model on the "impact" of Hours for Calvo parameter and Investment Adjustment Costs

that the change in sign in the NK model from both types of shocks becomes more pronounced than in the baseline calibration. Combining these results with those

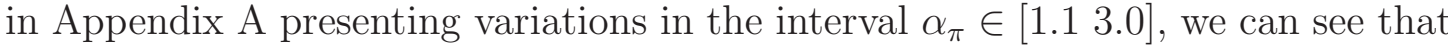
the Taylor coefficient is not crucial to determine the sign of the response of hours. ${ }^{26}$ Finally, Appendix E presents the IRF's for Hicks-neutral shocks, from which we had abstracted in the previous sections for simplicity.

\footnotetext{
${ }^{26}$ Taylor rule coefficients may, of course, change the hours-technology impact correlation in larger models (e.g., Galí, López-Salido and Vallés (2003)).
} 
The results from these exercises simply reflect, quantitatively, the mechanisms discussed in sections 6 and 7, and confirm that our results are robust to alternative policy and preferences specifications. It is worth stressing that we have also considered the effects of highly persistent and permanent shocks.

\section{A Time-Varying Correlation?}

Potentially, our study can help interpret some recent evidence that has suggested that technology-hours responses are time-varying. Fisher (2006), Fernald (2007), and Gambetti (2006), amongst others, report changes in the impact of technology shocks since the mid 1960s. Technology shocks appear to have a strong negative hours effect before the 1980s which then become almost insignificant (with another possible change in the mid-1990s). ${ }^{27}$ At the same time, the dynamics of the labor share in the US show a sharp decrease after the early 1980s and then a steady but slow increase until the mid 1990s. It is then likely that shocks that reduce the labor share were more prominent in the 1980s as suggested by theories of "directed technical change" (Acemoglu (2002)) if firms introduce technologies that reduce the use of the factors with a larger cost share. ${ }^{28}$

Thus, for an observed evolution of factor volumes, prices and income shares and preferred $\sigma$ - we may back out the sequence of labor- and capital-augmenting technology shocks consistent with that evolution. Accordingly, it is entirely plausible that the response of hours to technology shocks would be time-varying if the technology shocks are themselves time varying in their relative intensity. The value of the substitution elasticity will matter for the transmission of those shocks, and indeed may itself change over time, or, at the least, will differ across countries. ${ }^{29}$

In the same vein, if the introduction of certain production methods (e.g., IT technologies) changed the adjustment cost or depreciation rate of capital, this can have an impact on the response of hours as can easily be seen in the derivation of our threshold rules. Such changes modify the proportion of output devoted to saving, hence affecting the response of hours for given parameter values. ${ }^{30}$ Thus,

\footnotetext{
${ }^{27}$ Results differ by study but, in general, changes on the impact response can be observed around 1973, 1982 and also the first half of the 1990s

${ }^{28}$ McAdam and Willman (2008) develop a similar argument for the euro area.

${ }^{29}$ Muredduy and Strauch (2010) report that time-variations in sectoral substitution elasticities for the US and euro area map to changes in the share of ICT investment.We may also recall the Jones short-run/long run arguments relating to the substitution elasticity. Similarly, Yuhn (1991), following the "La Grandville (1989b) hypothesis", examined whether the high growth rate of east Asian countries was due, not to higher technical progress, but to a higher elasticity of substitution.

${ }^{30}$ Depreciation rates have trended upwards in recent years - see Evans (2000). This is compatible with the commonly-held view that the share of equipment in capital has increased while the share of structures has decreased and hence investment is characterized by shorter mean lives.
} 
our analysis may help interpret some of the time-varying evidence reported and provide a model-based foundation to such approaches.

\section{Conclusions}

We re-examined the impact of technology shocks on hours worked in business cycle models. The usual interpretation being that, in a Real Business Cycle (RBC) model, hours increase after a positive technology shock but initially fall in a New Keynesian (NK) one. This difference has been taken as a means of empirically discriminating between different theories of business-cycle fluctuations and remains a key controversy in macroeconomics.

Given the evidence, we argued that it is no longer defensible for business-cycle models to ignore non-unitary factor substitution and, by implication, factor-biased technical change; Cobb-Douglas is typically rejected by the data, and factor shares display important short- and medium-run fluctuations which, by definition, cannot be captured by unitary elasticity models.

Further, we demonstrated that introducing a CES supply with differing substitution possibility requires "normalization" for the calibration of dynamic general equilibrium models. By using normalized CES production functions, we re-stated that technology shocks can be either capital or labor saving. To give a flavor of the sensitivities involved, we showed that, in the standard RBC model, capitalaugmenting shocks yield positive hours responses, whilst labor-augmenting shocks can lead to responses of either sign. In the standard NK model, however, laboraugmenting shocks yield negative responses and capital-augmenting shocks were ambiguous.

However, more generally, we derived threshold rules for the determination of the technology-hours sign on impact. In each model and shock case, we showed that there exists some value of the elasticity of substitution whereby a given technologyhours impact changed sign. The key margins - other than the substitution elasticity itself and the factor bias of technology shocks - are the capital share, risk aversion, and the marginal propensity to consume. The latter essentially boils down to how persistent shocks are and the strength of investment adjustment costs. It is also important to stress that variations in these parameters prove to be much more important than variations in the degree of price rigidity when determining the sign of the response. These margins are then interpreted using a simple labor supply and demand framework.

We conclude that the impact of technology shocks on hours worked can hardly be taken as evidence in support of any particular business-cycle model. This is not to say that empirical evidence cannot discriminate between models only that concentrating on the hours response may lead to ambiguous or inconclusive 
evidence. Consequently, researchers may consider a wider class of discriminatory metrics and try to understand better the entire response space of their model.

Finally, our threshold rule may help shed light on possible time-variation in the hours-technology correlation. This is potentially a useful insight since much of the literature appears motivated by concerns of statistical fit rather than uncovering the underlying economic mechanisms. In our analysis, time variations may result from (i) time variation in the relative intensity of labor and capital augmenting shocks and (ii) time-variations in parameters (the substitution elasticity being prime among them).

These results open important new avenues for research. For instance, if it is not satisfactory for business-cycle models to assume unitary factor substitution, the practice implemented here of appropriately normalizing the supply side for calibration of DSGE models should become standard and related sensitivities analyzed. Moreover, although theoretical, our results can have empirical implications. We might, for instance, be able to exploit changes in factor income shares to identify different sorts of technology shocks in modeling and SVAR analysis. The models presented here can also serve as a benchmark to study the business cycle properties of movements in factor shares.

\section{References}

Acemoglu, Daron. 2002. "Directed technical change." Review of Economic Studies, 69: 781-809.

Acemoglu, Daron. 2009. Introduction to Modern Economic Growth. MIT Press.

Alexopoulos, Michelle. 2010. "Read all about it! What happens following a technology shock?" American Economic Review, forthcoming.

Arrow, Kenneth J., Hollis B. Chenery, Bagicha S. Minhas, and Robert M. Solow. 1961. "Capital-labor substitution and economic efficiency." Review of Economics and Statistics, 43(3): 225-250.

Barro, Robert J., and Xavier Sala-i-Martin. 2004. Economic Growth. Cambridge, MA.:2nd Edition, MIT Press.

Basu, Susanto, John G. Fernald, and Miles S. Kimball. 2006. "Are technology improvements contractionary?" American Economic Review, 96: 1418-1448.

Beach, Earl F. 1971. "Hicks on Ricardo on Machinery." Economic Journal, 81: 916-922.

Blanchard, Olivier J. 1997. "The Medium Run." Brookings Papers on Economic Activity, 2: 89-158. 
Chari, V. V., Patrick J. Kehoe, and Ellen R. McGrattan. 2008. "New Keynesian Models: Not Yet Useful for Policy Analysis." American Economic Journal: Macroeconomics, 1(1): 24-66.

Chirinko, Robert S. 2008. "Sigma: The Long and Short of It." Journal of Macroeconomics, 30(2): 671-686.

Christiano, Lawrence J., Martin Eichenbaum, and Charles L. Evans. 2005. "Nominal Rigidities and the Dynamic Effects of a Shock to Monetary Policy." Journal of Political Economy, 113(1): 1-45.

Christiano, Lawrence J., Martin Eichenbaum, and Robert Vigfusson. 2003. "What Happens After a Technology Shock?" National Bureau of Economic Research NBER Working Papers 9819.

Coenen, Günter, Peter McAdam, and Roland Straub. 2008. "Tax reform and labour-market performance in the euro area: a simulation-based analysis using the New Area-Wide Model." Journal of Economic Dynamics and Control, $32(8): 2543-2583$.

Dedola, Luca, and Stefano Neri. 2007. "What does a technology shock do? A VAR analysis with model-based sign restrictions." Journal of Monetary Economics, 54(2): 512-549.

Diamond, Peter A., and Daniel McFadden. 1965. "Identification of the Elasticity of Substitution and the bias of Technical Change: An Impossibility Theorem." Working Paper No. 62, University of California Berkeley.

Diamond, Peter A., Daniel McFadden, and Miguel Rodriguez. 1978. "Measurement of the elasticity of substitution and bias of technical change." In Production Economics, Vol. 2. , ed. Melvyn Fuss and Daniel McFadden, 125147. Amsterdam and North Holland.

Evans, Paul. 2000. "Stylized Facts and their implications for Growth Theory." Mimeo, Ohio State University.

Fernald, John G. 2007. "Trend breaks, long-run restrictions, and contractionary technology improvements." Journal of Monetary Economics, 54(8): 2467-2485.

Fernández-Villaverde, Jesús. 2010. "The Econometrics of DSGE Models." Journal of the Spanish Economic Association, 1(1): 3-49.

Fisher, Jonas D. M. 2006. "The Dynamic Effects of Neutral and InvestmentSpecific Technology Shocks." Journal of Political Economy, 114(3): 413-451.

Francis, Neville, and Valerie A. Ramey. 2005. "Is the technology-driven real business cycle hypothesis dead? Shocks and aggregate fluctuations revisited." Journal of Monetary Economics, 52(8): 1379-1399.

Francis, Neville, Michael T. Owyang, and Athena T. Theodorou. 2003. "The use of long-run restrictions for the identification of technology shocks." Federal Reserve Bank of St. Louis Review, 85(6): 53-66. 
Galí, Jordi. 1999. "Technology, Employment, and the Business Cycle: Do Technology Shocks Explain Aggregate Fluctuations?" American Economic Review, 89(1): 249-271.

Galí, Jordi. 2008. Monetary Policy, Inflation and the Business Cycle. Princeton University Press.

Galí, Jordi, and Luca Gambetti. 2009. "On the Sources of the Great Moderation." American Economic Journal: Macroeconomics, 1(1): 26-57.

Galí, Jordi, and Pau Rabanal. 2004. "Technology Shocks and Aggregate Fluctuations: How Well Does the RBC Model Fit Postwar U.S. Data?" NBER Macroeconomics Annual, 225-288.

Galí, Jordi, J. David López-Salido, and Javier Vallés. 2003. "Technology shocks and monetary policy: Assessing the Fed's performance." Journal of Monetary Economics, 50: 723-743.

Gambetti, Luca. 2006. "Time-Varying Dynamics Matter: Technology Shocks and the Response of Hours Worked." Mimeo.

Hicks, John R. 1969. A Theory of Economic History. Oxford University Press.

Jones, Charles I. 2003. "Growth, Capital Shares, and a New Perspective on Production Functions." mimeo, Stanford University.

Jones, Charles I. 2005. "The Shape of Production Functions and the Direction of Technical Change." Quarterly Journal of Economics, 120(2): 517-549.

Juillard, Michel. 2009. "Dynare Manual." Available at http: //www. dynare.org.

Klump, Rainer, and Olivier de La Grandville. 2000. "Economic growth and the elasticity of substitution: Two theorems and some suggestions." American Economic Review, 90: 282-291.

Klump, Rainer, Peter McAdam, and Alpo Willman. 2007. "Factor Substitution and Factor Augmenting Technical Progress in the US." Review of Economics and Statistics, 89(1): 183-92.

La Grandville, Olivier de. 1989a. "Curvature and the elasticity of substitution: Straightening it out." Journal of Economics, 66(1): 23-34.

La Grandville, Olivier de. 1989b. "In Quest of the Slutzky Diamond." American Economic Review, 79: 468-481.

La Grandville, Olivier de. 2009. Economic Growth: A Unified Approach. Cambridge University Press.

León-Ledesma, Miguel A., Peter McAdam, and Alpo Willman. 2010. "Identifying the Elasticity of Substitution with Biased Technical Change." American Economic Review, 100(4): 1330-1357. 
Levine, Paul, Peter McAdam, and Joseph G. Pearlman. 2008. "Quantifying and Sustaining Welfare Gains from Monetary Commitment." Journal of Monetary Economics, 55(2): 1253-78.

McAdam, Peter, and Alpo Willman. 2008. "Medium Run Redux." Working Paper No. 915, European Central Bank.

Muredduy, Francesco, and Rolf Strauch. 2010. "Accounting for the Productivity Slowdown." Mimeo, European Central Bank.

Pesavento, Elena, and Barbara Rossi. 2005. "Do Technology Shocks Drive Hours Up Or Down? A Little Evidence From An Agnostic Procedure." Macroeconomic Dynamics, 9(04): 478-488.

Prescott, Edward C. 1986. "Theory Ahead of Business Cycle Measurement." Quarterly Review, Federal Reserve Bank of Minneapolis, Fall: 9-22.

Rotemberg, Julio J. 2003. "Stochastic Technical Progress, Smooth Trends, and nearly distinct Business Cycles." American Economic Review, 93(5): 1543-1559.

Schmitt-Grohé, Stephanie, and Martin Uribe. 2007. "Optimal simple and implementable monetary and fiscal rules." Journal of Monetary Economics, 54(6): 1702-1725.

Smets, Frank, and Rafael Wouters. 2007. "Shocks and Frictions in US Business Cycles: A Bayesian DSGE Approach." American Economic Review, 97(3): 586-606.

Tovar, Camilo. 2008. "DSGE models and central banks." Working Papers No 258, Bank for International Settlements.

Uhlig, Harald. 2004. "Do Technology Shocks Lead to a Fall in Total Hours Worked?" Journal of the European Economic Association, 2(2-3): 361-371.

Whelan, Karl T. 2009. "Technology shocks and hours worked: Checking for robust conclusions." Journal of Macroeconomics, 31(2): 231-239.

Wicksell, Knut. 1911. Lectures on Political Economy. London: G. Routledge. 2nd edn, trans. E. Classen, 2 vols, 1934-5.

Yuhn, Ky-hyang. 1991. "Economic Growth, Technical Change Biases, and the Elasticity of Substitution: A Test of the De La Grandville Hypothesis." Review of Economics and Statistics, 73(2): 340-346. 


\section{Technical Appendices}

\section{A Sensitivity graphs}



(a) Sensitivity for $\rho$ with $\sigma=0.4$, RBC Model

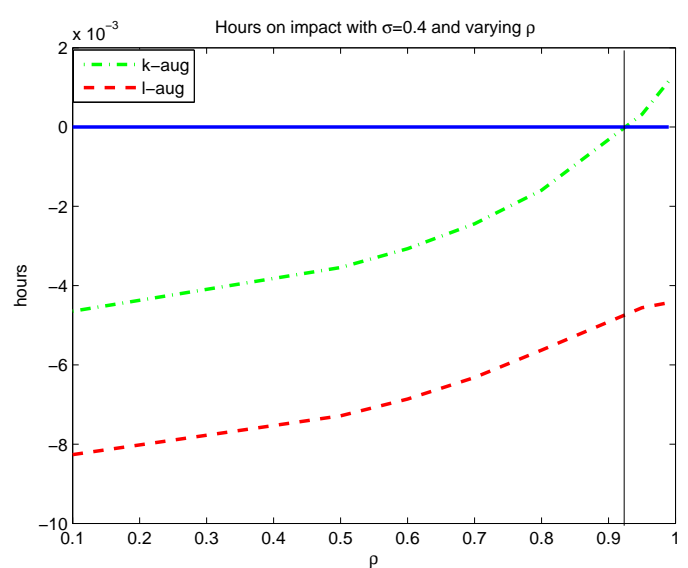

(c) Sensitivity for $\rho$ with $\sigma=0.4$, NK Model

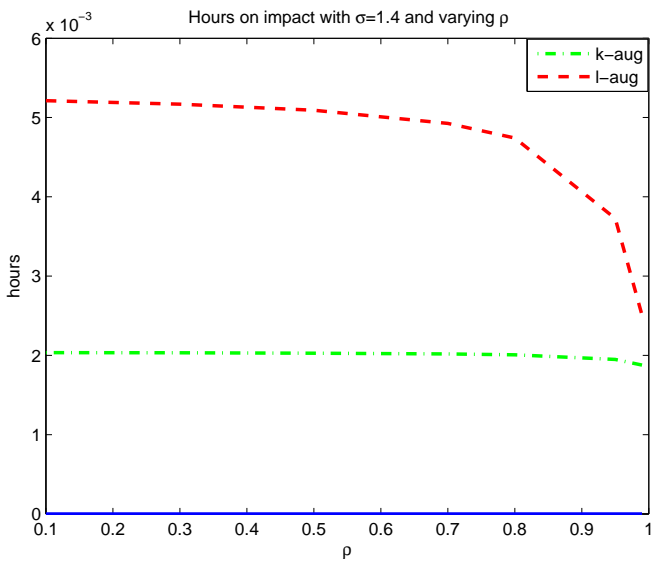

(b) Sensitivity for $\rho$ with $\sigma=1.4$, RBC Model

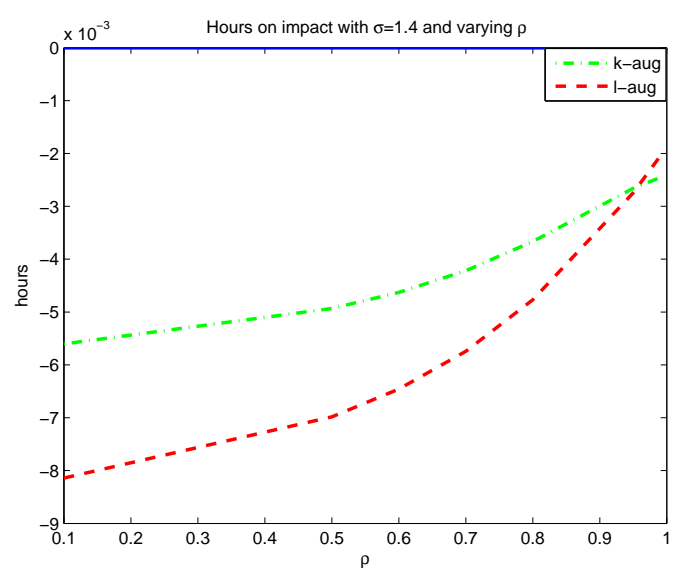

(d) Sensitivity for $\rho$ with $\sigma=1.4$, NK Model

Figure 11: Sensitivity analysis for $\rho$ 


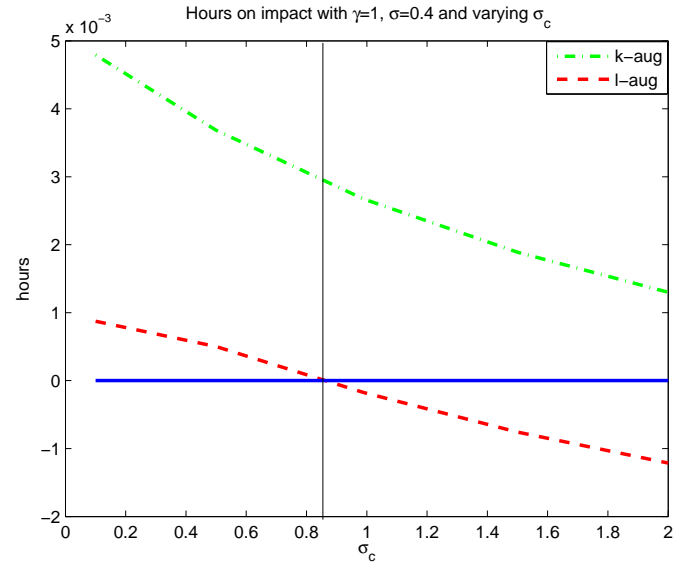

(a) Sensitivity for $\sigma_{c}$ with $\sigma=0.4$, RBC Model

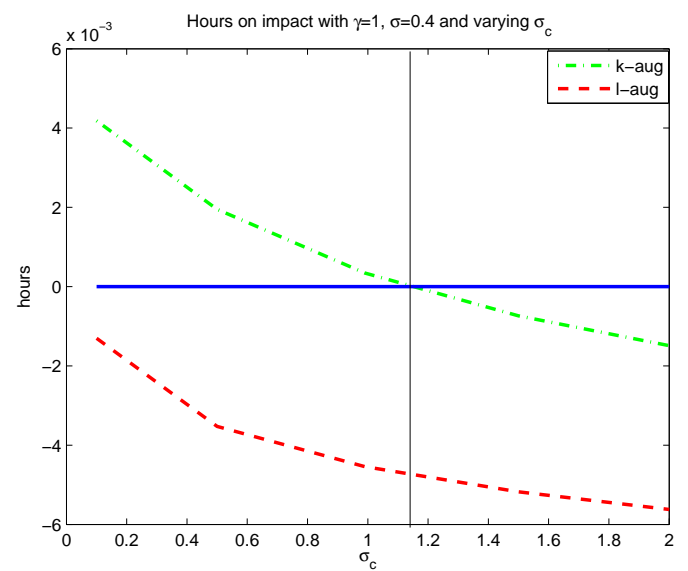

(c) Sensitivity for $\sigma_{c}$ with $\sigma=0.4$, NK Model

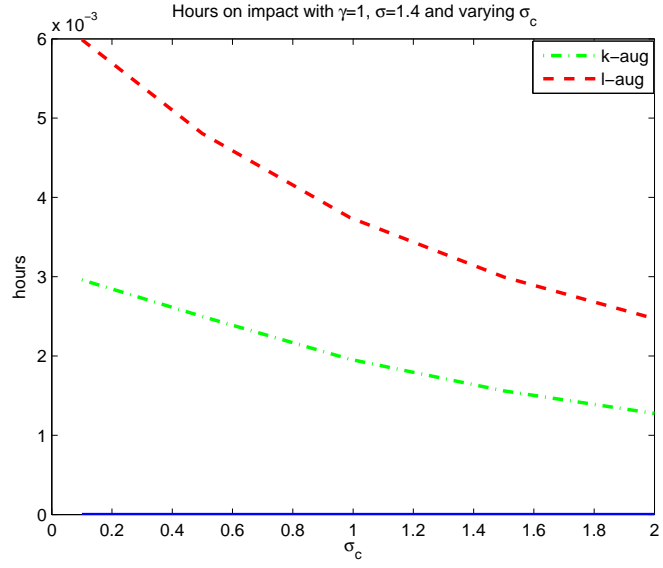

(b) Sensitivity for $\sigma_{c}$ with $\sigma=1.4, \mathrm{RBC}$ Model

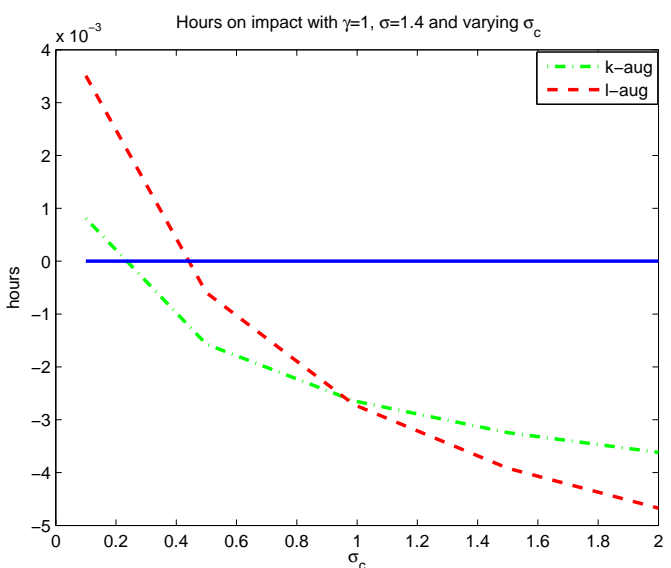

(d) Sensitivity for $\sigma_{c}$ with $\sigma=1.4$, NK Model

Figure 12: Sensitivity analysis for $\sigma_{c}$ 


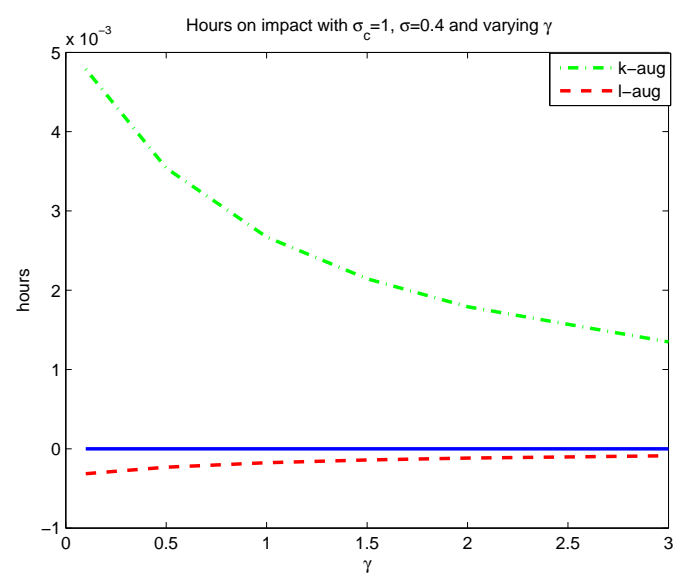

(a) Sensitivity for $\gamma$ with $\sigma=0.4$, RBC Model

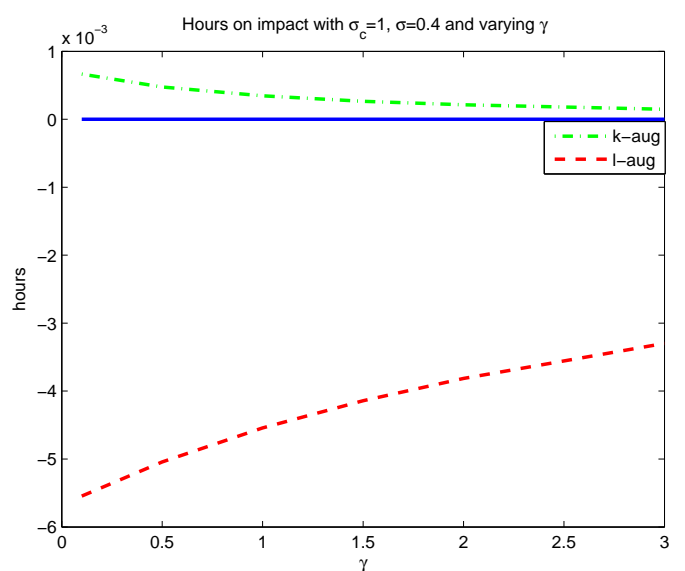

(c) Sensitivity for $\gamma$ with $\sigma=0.4$, NK Model

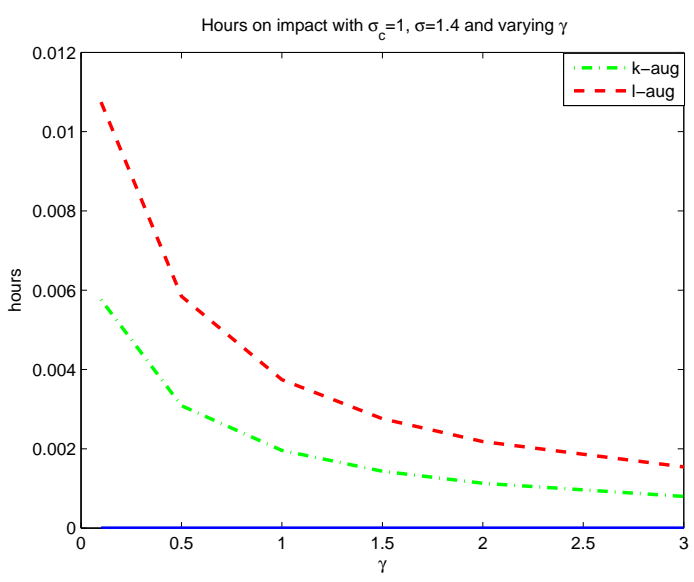

(b) Sensitivity for $\gamma$ with $\sigma=1.4$, RBC Model

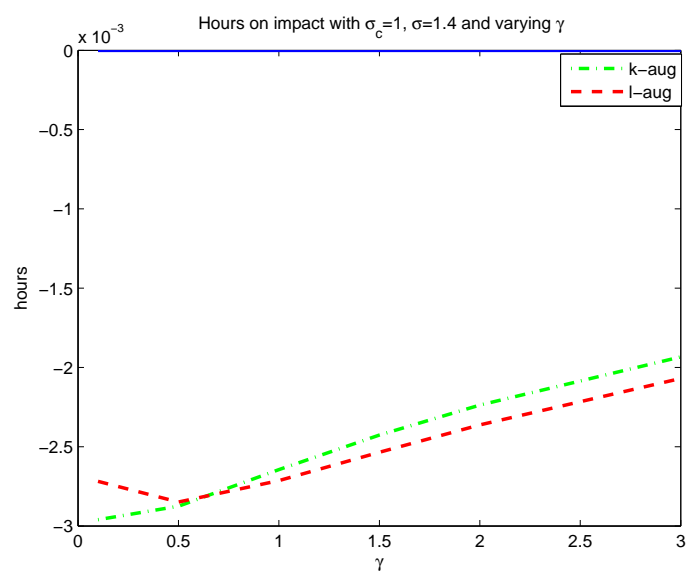

(d) Sensitivity for $\gamma$ with $\sigma=1.4$, NK Model

Figure 13: Sensitivity analysis for $\gamma$ 


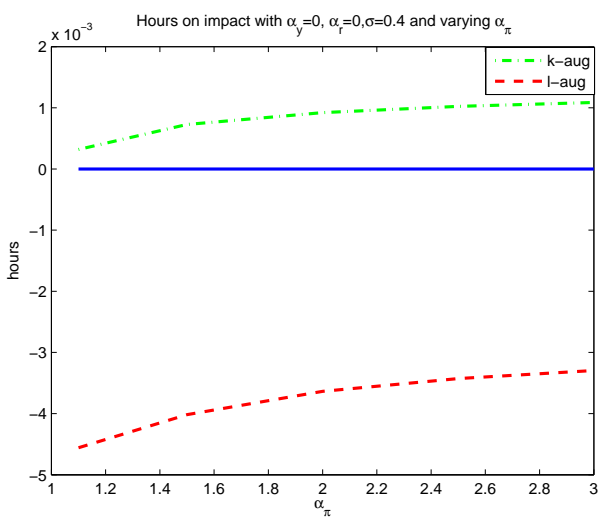

(a) Sensitivity for $\alpha_{\pi}$ when $\sigma=0.4$

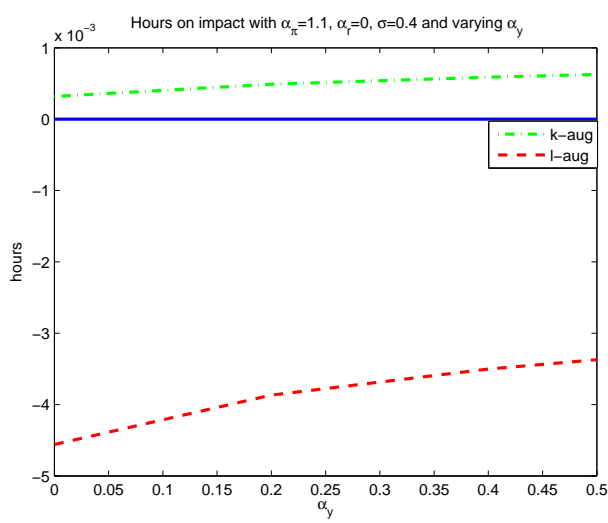

(c) Sensitivity for $\alpha_{y}$ when $\sigma=0.4$

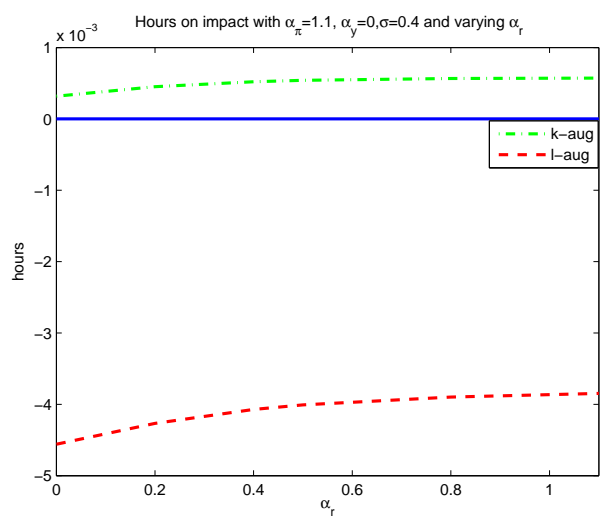

(e) Sensitivity for $\alpha_{r}$ when $\sigma=0.4$

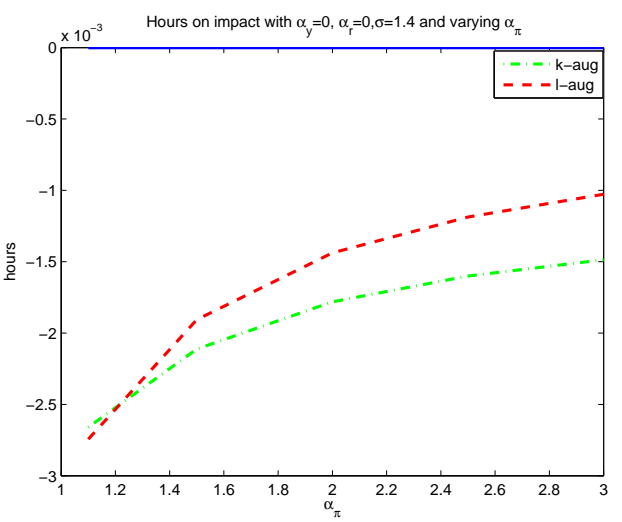

(b) Sensitivity for $\alpha_{\pi}$ when $\sigma=1.4$

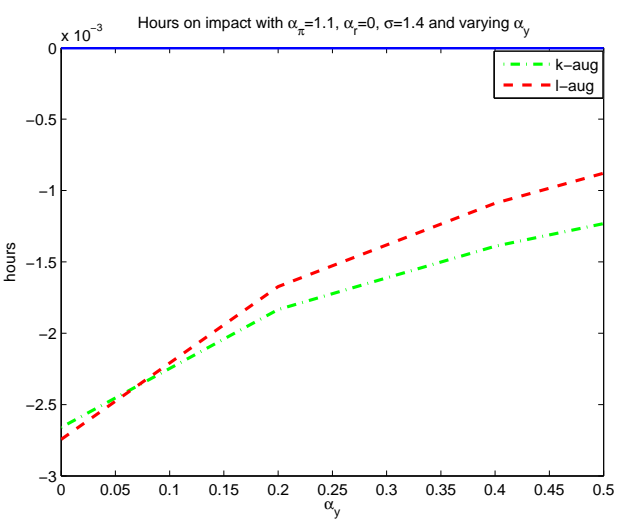

(d) Sensitivity for $\alpha_{y}$ when $\sigma=1.4$



(f) Sensitivity for $\alpha_{r}$ when $\sigma=1.4$

Figure 14: Sensitivity analysis in the NK model for monetary policy parameters 


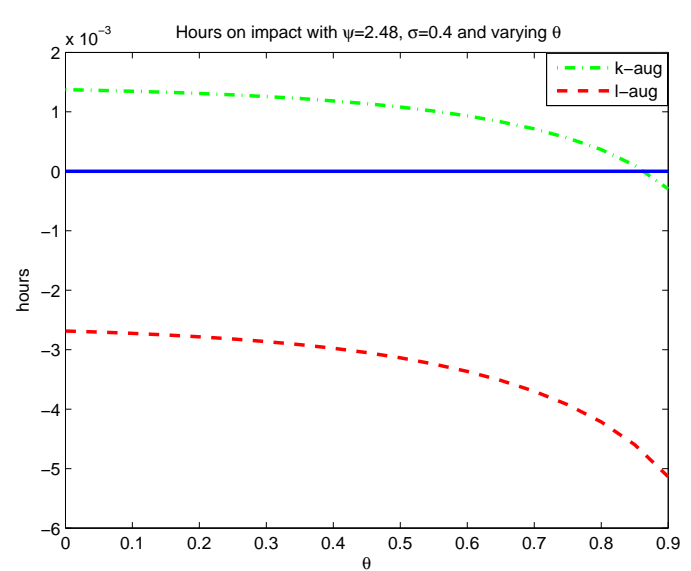

(a) Sensitivity for $\theta$ with $\sigma=0.4$, NK Model

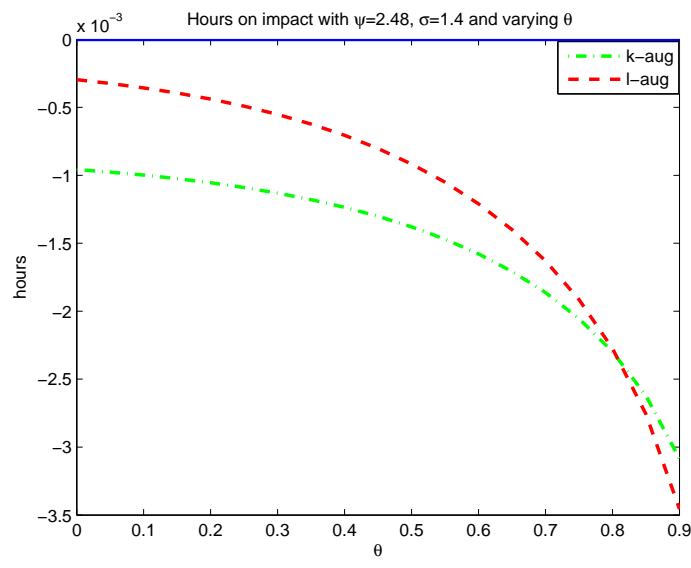

(b) Sensitivity for $\theta$ with $\sigma=1.4$, NK Model

Figure 15: Sensitivity analysis for $\theta$

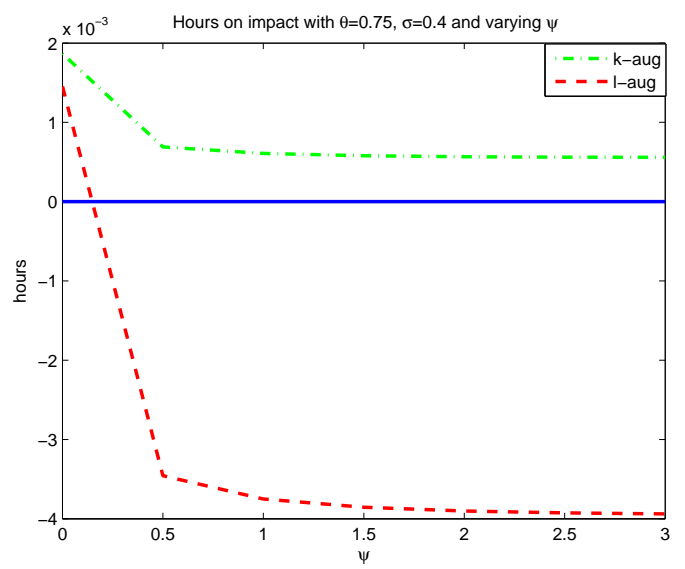

(a) Sensitivity for $\psi$ with $\sigma=0.4$, NK Model



(b) Sensitivity for $\psi$ with $\sigma=1.4$, NK Model

Figure 16: Sensitivity analysis for $\psi$ 


\section{B Threshold with non-separable preferences}

In the non-separable case, we define the utility function as,

$$
U\left(C_{t}, H_{t}\right)=\frac{\left[C_{t}^{\gamma}\left(T-H_{t}\right)^{1-\gamma}\right]^{1-\sigma_{c}}}{1-\sigma_{c}}
$$

where $T$ represents the total number of hours available in each period and thus $\Re=\frac{T-H}{H}$ represents the ratio of non-market-to-market activities. Corresponding to the steady state $H_{0}=1$, which we used earlier, a natural choice of $T$ would be around 3 , i.e. $\Re \approx 2$. Note, we could just as easily have set $T=1$ as commonly done, although for familiarity with our earlier case, we chose $H_{0}=1$. This yields the following labor supply equation (in log-difference form):

$$
d \log w_{t}=-d \log \left(T-H_{t}\right)+d \log C_{t} .
$$

The labor demand equation is obviously not affected by the change in the utility function. Around the steady state, $\Re=\frac{T-H}{H}$ and hence the growth of $w_{t}$ can be expressed as:

$$
d \log w_{t}=\frac{1}{\Re} d \log \left(T-H_{t}\right)+d \log C_{t} .
$$

This implies that the expression for the labor supply schedule (equation (65) in the main text) now becomes:

$$
d \log H_{t}=\frac{1}{1 / \Re+\frac{m p c t}{a p c} \phi\left(1-\alpha_{0}\right)}\left[d \log w_{t}-\frac{m p c_{t}}{a p c} \phi\left(\alpha_{0} d z_{t}^{K}+\left(1-\alpha_{0}\right) d z_{t}^{H}\right)\right] .
$$

It is clear that the rest of the analysis in the main text then remains the same, as this case of utility function is simply a limiting case of the separable utility function with $\sigma_{c}=1$ and $\gamma=1 / \Re{ }^{1}$ Hence, the new general threshold rule now

\footnotetext{
${ }^{1}$ This is logical as this form of utility function goes back to Prescott (1986) and is restricted to display both constant intertemporal and unit intra-temporal elasticities of substitution. This function implies an elasticity of substitution between consumption and leisure of 1 and is consistent with the observation that leisure per capita has shown virtually no secular trend. $1 / \sigma_{c}>0$ is the elasticity of substitution between different date composite commodities $C_{t}^{\gamma}\left(1-H_{t}\right)^{1-\gamma}$ where $\gamma$ is the consumption share parameter.
} 
becomes:

$$
\begin{array}{llll}
\frac{d \log H_{t}}{d z_{t}^{H}}>0 & \text { if } & \sigma>\frac{\alpha_{0}}{1+\frac{d \log m c_{t}}{d z_{t}^{H}}-\frac{m p c_{t}}{a p c} \phi\left(1-\alpha_{0}\right)} . \\
\frac{d \log H_{t}}{d z_{t}^{K}}>0 & \text { if } & \sigma<\frac{\alpha_{0}}{\frac{m p c_{t}}{a p c} \phi \alpha_{0}-\frac{d \log m c_{t}}{d z_{t}^{K}}}
\end{array}
$$




\section{Numerical simulation tables}

The general threshold conditions (60) and (61) can be written in the following form form:

$$
\begin{aligned}
& \frac{d \log H_{t}}{d z_{t}^{H}}>0 \quad \text { if } \underbrace{m p c_{t}-\frac{a p c}{\phi \sigma_{c}\left(1-\alpha_{0}\right)} \frac{d \log m c_{t}}{d z_{t}^{H}}}_{m p c^{-} \chi m c_{t}}<\frac{a p c}{\phi\left(1-\alpha_{0}\right) \sigma_{c}}\left(1-\frac{\alpha_{0}}{\sigma}\right) ; \\
& \frac{d \log H_{t}}{d z_{t}^{K}}>0 \quad \text { if } \underbrace{m p c_{t}-\frac{a p c}{\phi \sigma_{c} \alpha_{0}} \frac{d \log m c_{t}}{d z_{t}^{K}}}_{m p c_{t}-v m c_{t}}<\frac{a p c}{\phi \sigma_{c} \sigma} .
\end{aligned}
$$

Hence, these two conditions can be described with help of two curves. The right hand sides of both (C.1) and (C.2) are determined solely by the parameters of the model. The functional forms of the right hand sides with respect to $\sigma$ are hyperbolas. The hyperbola corresponding to the labor-augmenting threshold condition increases from minus infinity to its horizontal asymptote $\frac{a p c}{\phi\left(1-\alpha_{0}\right) \sigma_{c}}$, when $\sigma$ grows from zero to infinity. The hyperbola related to the capital augmenting threshold condition is downward sloping and it falls from infinity to zero, when $\sigma$ grows from zero to infinity. The left-hand sides, in turn, represent the endogenous reactions of the marginal propensity to consume $\left(m p c_{t}\right)$ and the real marginal cost $\left(m c_{t}\right)$ and, hence, are known a priori only in some special cases as depicted in the main text.

In the RBC-model real marginal costs $\left(m c_{t}\right)$ do not react to shocks and, hence, conditions (C.1) and (C.2) simplify to,

$$
\begin{array}{ll}
\frac{d \log H_{t}}{d z_{t}^{H}}>0 \quad \text { if } \quad m p c_{t}<\frac{a p c}{\phi\left(1-\alpha_{0}\right) \sigma_{c}}\left(1-\frac{\alpha_{0}}{\sigma}\right) \\
\frac{d \log H_{t}}{d z_{t}^{K}}>0 \quad \text { if } \quad m p c_{t}<\frac{a p c}{\phi \sigma_{c} \sigma} .
\end{array}
$$

Tables A1-A5 present the $m p c_{t}$ and $m p c_{t^{-}} \chi m c_{t}$ reactions to a labor augmenting shock, when $\sigma$ takes the range of values $(0.3,0.9,1.4,2,10)$ with different combinations of other model parameters. As long-as the $m p c_{t}$ (RBC-model) or $m p c_{t^{-}} \chi m c_{t}$ (NK-model) response is below (above) the right hand side value of the hyperbola with the same parameter configuration, then the hours-response to the shock is positive (negative). This is indicated by $(+)$ or $(-)$ signs in the tables. Tables A6-A10 present corresponding results for capital augmenting shocks. 
Table A1. RBC: Impact responses of $m p c_{t}$ to a labor augmenting shock, the values of the hyperbola $\frac{a p c}{\left(1-\alpha_{0}\right) \sigma_{c}}\left(1-\frac{\alpha_{0}}{\sigma}\right)$ and the sign of $d H / d z^{H},\left(\right.$ apc $=0.718, \Psi=0$ and $\left.\alpha_{0}=0.4\right)$.

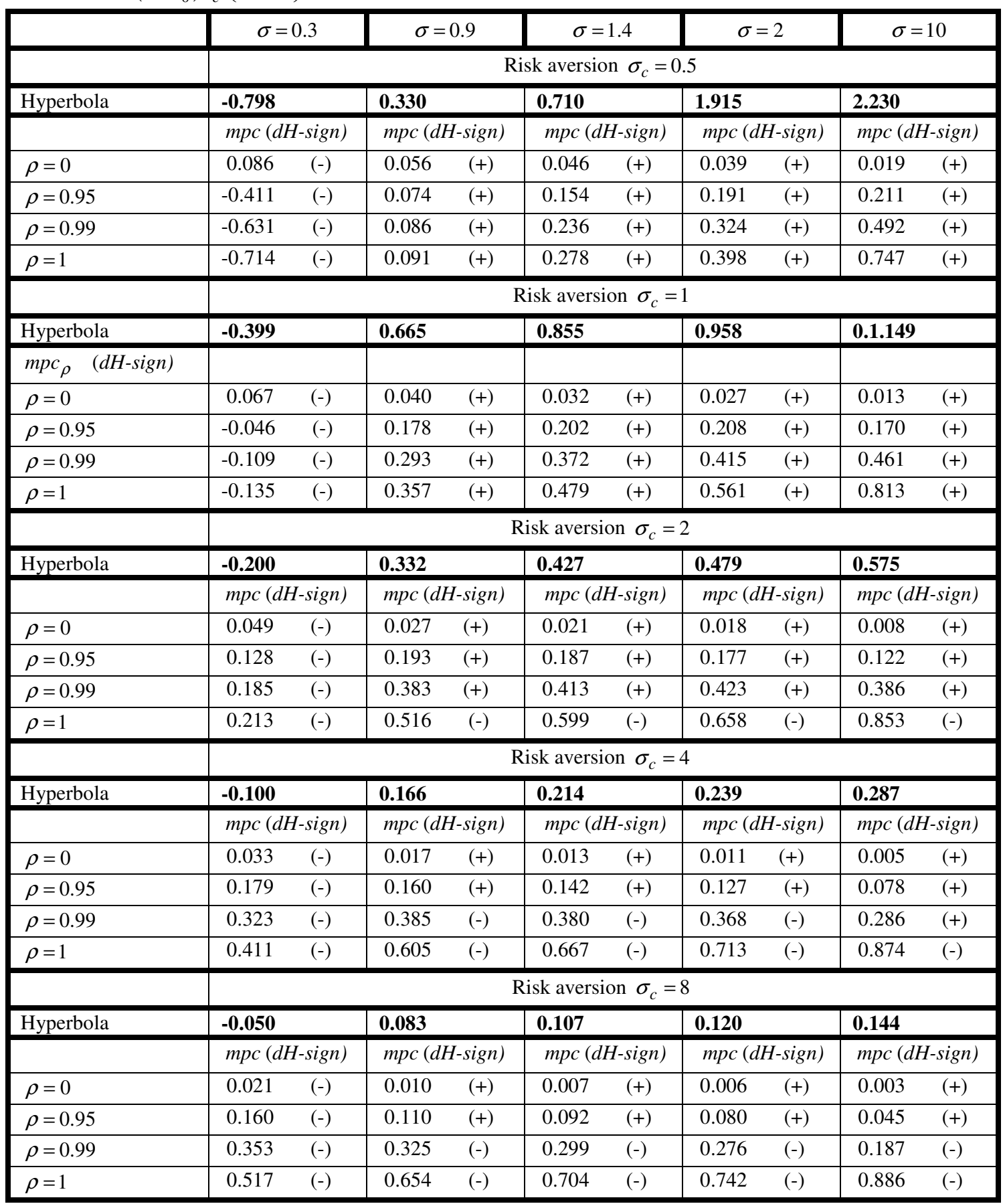


Table A2. Generalized RBC ( Finite $\eta$ ): Impact responses of $m p c_{t}$ to a labor augmenting shock, the values of the hyperbola $\frac{a p c}{\left(1-\alpha_{0}\right) \sigma_{c}}\left(\frac{\eta-1}{\eta}\right)\left(1-\frac{\alpha_{0}}{\sigma}\right)$ and the sign of $d H / d z^{H}$, $\left(a p c=0.718, \Psi=0\right.$ and $\left.\alpha_{0}=0.4\right)$.

\begin{tabular}{|c|c|c|c|c|c|}
\hline & $\sigma=0.3$ & $\sigma=0.9$ & $\sigma=1.4$ & $\sigma=2$ & $\sigma=10$ \\
\hline & \multicolumn{5}{|c|}{ Risk aversion $\sigma_{c}=2$, price elasticity $\eta=\infty$} \\
\hline \multirow[t]{2}{*}{ Hyperbola } & -0.200 & 0.332 & 0.427 & 0.479 & 0.575 \\
\hline & $m p c(d H$-sign $)$ & $m p c(d H$-sign $)$ & $m p c(d H-s i g n)$ & $m p c(d H$-sign $)$ & $m p c(d H$-sign $)$ \\
\hline$\rho=0$ & 0.049 & 0.027 & 0.021 & 0.018 & 0.008 \\
\hline$\rho=0.95$ & 0.128 & 0.193 & 0.187 & 0.177 & 0.122 \\
\hline$\rho=0.99$ & 0.185 & 0.383 & 0.413 & 0.423 & 0.386 \\
\hline \multirow[t]{2}{*}{$\rho=1$} & 0.213 & 0.516 & 0.599 & 0.658 & 0.853 \\
\hline & \multicolumn{5}{|c|}{ Risk aversion $\sigma_{c}=2$, price elasticity $\eta=10$} \\
\hline \multirow[t]{2}{*}{ Hyperbola } & -0.180 & 0.299 & 0.385 & 0.431 & 0.517 \\
\hline & $m p c(d H$-sign $)$ & $m p c(d H-s i g n)$ & $m p c(d H-s i g n)$ & $m p c(d H$-sign $)$ & $m p c(d H$-sign $)$ \\
\hline$\rho=0$ & 0.050 & 0.028 & 0.022 & 0.018 & 0.009 \\
\hline$\rho=0.95$ & 0.179 & 0.217 & 0.206 & 0.193 & 0.134 \\
\hline$\rho=0.99$ & 0.269 & 0.430 & 0.451 & 0.457 & 0.415 \\
\hline \multirow[t]{2}{*}{$\rho=1$} & 0.312 & 0.575 & 0.650 & 0.703 & 0.878 \\
\hline & \multicolumn{5}{|c|}{ Risk aversion $\sigma_{c}=2$, price elasticity $\eta=2$} \\
\hline \multirow[t]{2}{*}{ Hyperbola } & -0.100 & 0.166 & 0.214 & 0.239 & 0.287 \\
\hline & $m p c(d H$-sign $)$ & $m p c(d H-s i g n)$ & $m p c(d H-s i g n)$ & $m p c(d H$-sign $)$ & $m p c(d H-s i g n)$ \\
\hline$\rho=0$ & 0.059 & 0.032 & 0.025 & 0.022 & 0.013 \\
\hline$\rho=0.95$ & 0.383 & 0.319 & 0.288 & 0.265 & 0.198 \\
\hline$\rho=0.99$ & 0.584 & 0.611 & 0.603 & 0.593 & 0.541 \\
\hline$\rho=1$ & 0.675 & 0.794 & 0.833 & 0.862 & 0.954 \\
\hline & & Risk aversi & $\sigma_{c}=1$, price ela & ticity $\eta=10$ & \\
\hline Hyperbola & -0.359 & 0.598 & 0.769 & 0.862 & 1.034 \\
\hline & $m p c(d H$-sign $)$ & $m p c(d H$-sign $)$ & $m p c(d H$-sign $)$ & $m p c(d H$-sign $)$ & $m p c(d H$-sign $)$ \\
\hline$\rho=0$ & 0.069 & 0.042 & 0.033 & 0.028 & 0.014 \\
\hline$\rho=0.95$ & 0.038 & 0.217 & 0.233 & 0.233 & 0.188 \\
\hline$\rho=0.99$ & 0.021 & 0.362 & 0.428 & 0.463 & 0.496 \\
\hline$\rho=1$ & 0.014 & 0.439 & 0.547 & 0.620 & 0.845 \\
\hline
\end{tabular}


Table A3. Generalized RBC ( $\Psi \neq 0$ ): Impact responses of $m p c_{t}$ to a labor augmenting shock, the values of the hyperbola $\frac{a p c}{\left(1-\alpha_{0}\right) \sigma_{c}}\left(1-\frac{\alpha_{0}}{\sigma}\right)$ and the sign of $d H / d z^{H},(a p c=0.718$ and $\left.\alpha_{0}=0.4\right)$

\begin{tabular}{|c|c|c|c|c|c|}
\hline & $\sigma=0.3$ & $\sigma=0.9$ & $\sigma=1.4$ & $\sigma=2$ & $\sigma=10$ \\
\hline Hyperbola & -0.200 & 0.332 & 0.427 & 0.479 & 0.575 \\
\hline & \multicolumn{5}{|c|}{ Risk aversion $\sigma_{c}=2$, Adjustment costs of investment $\Psi=0$} \\
\hline & $m p c(d H$-sign $)$ & $m p c(d H$-sign $)$ & $m p c(d H$-sign $)$ & $m p c(d H$-sign $)$ & $m p c(d H-$ sign $)$ \\
\hline$\rho=0$ & 0.049 & 0.027 & 0.021 & 0.018 & 0.008 \\
\hline$\rho=0.95$ & 0.128 & 0.193 & 0.187 & 0.177 & 0.122 \\
\hline$\rho=0.99$ & 0.185 & 0.383 & 0.413 & 0.423 & 0.386 \\
\hline \multirow[t]{3}{*}{$\rho=1$} & 0.213 & 0.516 & 0.599 & 0.658 & 0.853 \\
\hline & \multicolumn{5}{|c|}{ Risk aversion $\sigma_{c}=2$, Adjustment costs of investment $\Psi=0.1$} \\
\hline & $m p c(d H$-sign $)$ & $m p c(d H$-sign $)$ & $m p c(d H$-sign $)$ & $m p c(d H$-sign $)$ & $m p c(d H-s i g n)$ \\
\hline$\rho=0$ & 0.226 & 0.208 & 0.202 & 0.198 & 0.189 \\
\hline$\rho=0.95$ & 0.220 & 0.286 & 0.281 & 0.272 & 0.219 \\
\hline$\rho=0.99$ & 0.271 & 0.462 & 0.491 & 0.502 & 0.471 \\
\hline \multirow[t]{3}{*}{$\rho=1$} & 0.296 & 0.582 & 0.659 & 0.713 & 0.880 \\
\hline & \multicolumn{5}{|c|}{ Risk aversion $\sigma_{c}=2$, Adjustment costs of investment $\Psi=1$} \\
\hline & $m p c(d H$-sign $)$ & $m p c(d H$-sign $)$ & $m p c(d H$-sign $)$ & $m p c(d H-$ sign $)$ & $m p c(d H-s i g n)$ \\
\hline$\rho=0$ & 0.665 & 0.654 & 0.650 & 0.648 & 0.641 \\
\hline$\rho=0.95$ & 0.492 & 0.540 & 0.537 & 0.529 & 0.484 \\
\hline$\rho=0.99$ & 0.517 & 0.661 & 0.684 & 0.693 & 0.672 \\
\hline \multirow[t]{3}{*}{$\rho=1$} & 0.534 & 0.744 & 0.797 & 0.832 & 0.934 \\
\hline & \multicolumn{5}{|c|}{ Risk aversion $\sigma_{c}=2$, Adjustment costs of investment $\Psi=2.5$} \\
\hline & $m p c(d H$-sign $)$ & $m p c(d H$-sign $)$ & $m p c(d H-$ sign $)$ & $m p c(d H-$ sign $)$ & $m p c(d H-$ sign $)$ \\
\hline$\rho=0$ & 0.816 & 0.810 & 0.808 & 0.806 & 0.802 \\
\hline$\rho=0.95$ & 0.625 & 0.663 & 0.660 & 0.654 & 0.616 \\
\hline$\rho=0.99$ & 0.637 & 0.750 & 0.768 & 0775 & 0.759 \\
\hline \multirow[t]{3}{*}{$\rho=1$} & 0.648 & 0.812 & 0.853 & 0.879 & 0.954 \\
\hline & \multicolumn{5}{|c|}{ Risk aversion $\sigma_{c}=2$, Adjustment costs of investment $\Psi=10$} \\
\hline & $m p c(d H$-sign $)$ & $m p c(d H$-sign $)$ & $m p c(d H-s i g n)$ & $m p c(d H-s i g n)$ & $m p c(d H-s i g n)$ \\
\hline$\rho=0$ & 0.940 & 0.938 & 0.937 & 0.937 & 0.936 \\
\hline$\rho=0.95$ & 0.795 & 0.817 & 0.816 & 0.813 & 0.791 \\
\hline$\rho=0.99$ & 0.789 & 0.858 & 0.869 & 0.874 & 0.865 \\
\hline$\rho=1$ & 0.792 & 0.893 & 0.917 & 0.932 & 0.975 \\
\hline
\end{tabular}


Table A4. NK ( $\Psi=0)$ : Impact responses of $m p c_{t}$ and $m p c_{t}-\chi \cdot m c_{t}$ to a labor augmenting shock, the values of the hyperbola $\frac{a p c}{\left(1-\alpha_{0}\right) \sigma_{c}}\left(\frac{\eta-1}{\eta}\right)\left(1-\frac{\alpha_{0}}{\sigma}\right)$ and the sign of $d H / d z^{H}$, $\left(a p c=0.718, \sigma_{c}=1 \eta=10, \alpha_{0}=0.4\right)$.

\begin{tabular}{|c|c|c|c|c|c|}
\hline & $\sigma=0.3$ & $\sigma=0.9$ & $\sigma=1.4$ & $\sigma=2$ & $\sigma=10$ \\
\hline Hyperbola & -0.359 & 0.598 & 0.769 & 0.862 & 1.034 \\
\hline & \multicolumn{5}{|c|}{ Calvo parameter $\theta=0.5$, Adjustment costs of investment $\Psi=0$} \\
\hline & $($ dH-sign $)$ & $($ dH-sign $)$ & $(d H$-sign $)$ & $m p c(d H$-sign $)$ & $($ dH-sign $)$ \\
\hline $\operatorname{mpc} \rho=0$ & 0.069 & 0.042 & 0.033 & 0.028 & 0.014 \\
\hline $\mathrm{mpc}-\chi \cdot \mathrm{mc} \rho=0$ & 0.074 & 0.045 & 0.036 & 0.030 & 0.014 \\
\hline $\operatorname{mpc} \rho=0.95$ & 0.038 & 0.213 & 0.229 & 0.231 & 0.188 \\
\hline $\mathrm{mpc}-\chi \cdot \mathrm{mc} \rho=0.95$ & -0.060 & 0.166 & 0.196 & 0.206 & 0.182 \\
\hline $\operatorname{mpc} \rho=0.99$ & 0.022 & 0.354 & 0.421 & 0.457 & 0.495 \\
\hline $\mathrm{mpc}-\chi \cdot \mathrm{mc} \rho=0.99$ & -0.081 & 0.309 & 0.390 & 0.435 & 0.490 \\
\hline $\operatorname{mpc} \rho=1$ & 0.016 & 0.430 & 0.538 & 0.612 & 0.842 \\
\hline \multirow[t]{3}{*}{$\mathrm{mpc}-\chi \cdot \mathrm{mc} \rho=1$} & -0.089 & 0.386 & 0.508 & 0.591 & 0.838 \\
\hline & \multicolumn{5}{|c|}{ Calvo parameter $\theta=0.75$, Adjustment costs of investment $\Psi=0$} \\
\hline & $(d H$-sign $)$ & $(d H$-sign $)$ & $($ dH-sign $)$ & $m p c(d H-s i g n)$ & $(d H-$ sign $)$ \\
\hline $\operatorname{mpc} \rho=0$ & 0.069 & 0.042 & 0.033 & 0.028 & 0.014 \\
\hline $\mathrm{mpc}-\chi \cdot \mathrm{mc} \rho=0$ & 0.100 & 0.063 & 0.50 & 0.040 & 0.017 \\
\hline $\operatorname{mpc} \rho=0.95$ & 0.041 & 0.197 & 0.215 & 0.219 & 0.185 \\
\hline $\mathrm{mpc}-\chi \cdot \mathrm{mc} \rho=0.95$ & -0.539 & -0.082 & 0.018 & 0.073 & 0.151 \\
\hline $\operatorname{mpc} \rho=0.99$ & 0.027 & 0.323 & 0.391 & 0.431 & 0.487 \\
\hline $\mathrm{mpc}-\chi \cdot \mathrm{mc} \rho=0.99$ & -0.578 & 0.055 & 0.207 & 0.298 & 0.458 \\
\hline $\operatorname{mpc} \rho=1$ & 0.021 & 0.391 & 0.499 & 0.576 & 0.829 \\
\hline $\mathrm{mpc}-\chi \cdot \mathrm{mc} \rho=1$ & -0.590 & 0.130 & 0.324 & 0.452 & 0.805 \\
\hline & $\mathrm{Ca}$ & parameter $\theta=0$ & , Adjustment $\mathrm{cc}$ & ts of investment & $=0$ \\
\hline & $($ dH-sign $)$ & $(d H$-sign $)$ & $(d H$-sign $)$ & $m p c(d H$-sign $)$ & $(d H$-sign $)$ \\
\hline $\operatorname{mpc} \rho=0$ & 0.069 & 0.042 & 0.033 & 0.028 & 0.014 \\
\hline $\mathrm{mpc}-\chi \cdot \mathrm{mc} \rho=0$ & 0.163 & 0.108 & 0.083 & 0.066 & 0.023 \\
\hline $\operatorname{mpc} \rho=0.95$ & 0.045 & 0.166 & 0.186 & 0.194 & 0.179 \\
\hline $\mathrm{mpc}-\chi \cdot \mathrm{mc} \rho=0.95$ & -1.740 & -0.693 & -0.422 & -0.259 & 0.072 \\
\hline $\operatorname{mpc} \rho=0.99$ & 0.034 & 0.267 & 0.333 & 0.377 & 0.469 \\
\hline mpc- $\chi \cdot \mathrm{mc} \rho=0.99$ & -1.816 & -0.556 & -0.231 & -0.032 & 0.379 \\
\hline $\operatorname{mpc} \rho=1$ & 0.031 & 0.322 & 0.423 & 0.503 & 0.798 \\
\hline $\mathrm{mpc}-\chi \cdot \mathrm{mc} \rho=1$ & -1.835 & -0.476 & 0.111 & 0.123 & 0.722 \\
\hline
\end{tabular}


Table A5. NK: Impact responses of $m p c_{t}$ and $m p c_{t}-\chi \cdot m c_{t}$ to a labor augmenting shock, the values of the hyperbola $\frac{a p c}{\left(1-\alpha_{0}\right) \sigma_{c}}\left(\frac{\eta-1}{\eta}\right)\left(1-\frac{\alpha_{0}}{\sigma}\right)$ and the sign of $d H / d z^{H}$, $\left(a p c=0.718, \sigma_{c}=1 \eta=10, \alpha_{0}=0.4\right)$.

\begin{tabular}{|c|c|c|c|c|c|}
\hline & $\sigma=0.3$ & $\sigma=0.9$ & $\sigma=1.4$ & $\sigma=2$ & $\sigma=10$ \\
\hline Hyperbola & -0.359 & 0.598 & 0.769 & 0.862 & 1.034 \\
\hline & \multicolumn{5}{|c|}{ Calvo parameter $\theta=0.75$, Adjustment costs of investment $\Psi=0$} \\
\hline & $(d H$-sign $)$ & $(d H$-sign $)$ & $(d H$-sign $)$ & $m p c(d H$-sign $)$ & $(d H-s i g n)$ \\
\hline $\operatorname{mpc} \rho=0$ & 0.069 & 0.042 & 0.033 & 0.028 & 0.014 \\
\hline $\mathrm{mpc}-\chi \cdot \mathrm{mc} \rho=0$ & 0.100 & 0.063 & 0.50 & 0.040 & 0.017 \\
\hline $\operatorname{mpc} \rho=0.95$ & 0.042 & 0.194 & 0.213 & 0.218 & 0.185 \\
\hline $\mathrm{mpc}-\chi \cdot \mathrm{mc} \rho=0.95$ & -0.538 & -0.084 & 0.017 & 0.072 & 0.151 \\
\hline mpc $\rho=0.99$ & 0.029 & 0.319 & 0.388 & 0.429 & 0.487 \\
\hline $\mathrm{mpc}-\chi \cdot \mathrm{mc} \rho=0.99$ & -0.575 & 0.050 & 0.205 & 0.296 & 0.458 \\
\hline $\operatorname{mpc} \rho=1$ & 0.024 & 0.386 & 0.495 & 0.574 & 0.829 \\
\hline \multirow[t]{3}{*}{$\mathrm{mpc}-\chi \cdot \mathrm{mc} \rho=1$} & -0.587 & 0.125 & 0.321 & 0.450 & 0.805 \\
\hline & \multicolumn{5}{|c|}{ Calvo parameter $\theta=0.75$, Adjustment costs of investment $\Psi=0.1$} \\
\hline & $($ dH-sign $)$ & $(d H$-sign $)$ & $(d H$-sign $)$ & $m p c(d H-$ sign $)$ & $(d H$-sign $)$ \\
\hline $\operatorname{mpc} \rho=0$ & 0.287 & 0.267 & 0.260 & 0.254 & 0.238 \\
\hline $\mathrm{mpc}-\chi \cdot \mathrm{mc} \rho=0$ & 1.315 & 1.492 & 1.529 & 1.550 & 1.590 \\
\hline mpc $\rho=0.95$ & 0.170 & 0.360 & 0.382 & 0.388 & 0.349 \\
\hline $\mathrm{mpc}-\chi \cdot \mathrm{mc} \rho=0.95$ & 0.357 & 0.662 & 0.717 & 0.746 & 0.789 \\
\hline mpc $\rho=0.99$ & 0.149 & 0.492 & 0.560 & 0.598 & 0.642 \\
\hline $\mathrm{mpc}-\chi \cdot \mathrm{mc} \rho=0.99$ & 0.315 & 0.681 & 0.750 & 0.788 & 0.849 \\
\hline $\operatorname{mpc} \rho=1$ & 0.141 & 0.558 & 0.659 & 0.724 & 0.900 \\
\hline \multirow[t]{3}{*}{$\mathrm{mpc}-\chi \cdot \mathrm{mc} \rho=1$} & 0.304 & 0.701 & 0.782 & 0.831 & 0.946 \\
\hline & \multicolumn{5}{|c|}{ Calvo parameter $\theta=0.75$, Adjustment costs of investment $\Psi=1$} \\
\hline & $(d H$-sign $)$ & (dH-sign) & (dH-sign) & (dH-sign) & $($ dH-sign $)$ \\
\hline $\operatorname{mpc} \rho=0$ & 0.718 & 0.706 & 0.701 & 0.698 & 0.688 \\
\hline $\mathrm{mpc}-\chi \cdot \mathrm{mc} \rho=0$ & 2.103 & 2.222 & 2.246 & 2.258 & 2.280 \\
\hline mpc $\rho=0.95$ & 0.492 & 0.625 & 0.638 & 0.641 & 0.610 \\
\hline $\mathrm{mpc}-\chi \cdot \mathrm{mc} \rho=0.95$ & 0.938 & 1.046 & 1.064 & 1.074 & 1.090 \\
\hline $\operatorname{mpc} \rho=0.99$ & 0.458 & 0.704 & 0.747 & 0.770 & 0.794 \\
\hline $\mathrm{mpc}-\chi \cdot \mathrm{mc} \rho=0.99$ & 0.882 & 0.987 & 1.001 & 1.008 & 1.020 \\
\hline $\operatorname{mpc} \rho=1$ & 0.447 & 0.747 & 0.809 & 0.848 & 0.947 \\
\hline $\mathrm{mpc}-\chi \cdot \mathrm{mc} \rho=1$ & 0.869 & 0.973 & 0.985 & 0.990 & 0.999 \\
\hline & Calv & ameter $\theta=0$. & djustment cos & of investment $\Psi$ & $=2.5$ \\
\hline & $(d H$-sign $)$ & (dH-sign) & $(d H$-sign $)$ & $m p c(d H$-sign $)$ & $(d H$-sign $)$ \\
\hline $\operatorname{mpc} \rho=0$ & 0.849 & 0.841 & 0.839 & 0.837 & 0.831 \\
\hline $\mathrm{mpc}-\chi \cdot \mathrm{mc} \rho=0$ & 2.267 & 2.382 & 2.404 & 2.417 & 2.439 \\
\hline $\mathrm{mpc} \rho=0.95$ & 0.644 & 0.737 & 0.746 & 0.748 & 0.726 \\
\hline $\mathrm{mpc}-\chi \cdot \mathrm{mc} \rho=0.95$ & 1.054 & 1.115 & 1.125 & 1.130 & 1.141 \\
\hline mpc $\rho=0.99$ & 0.607 & 0.787 & 0.818 & 0.834 & 0.852 \\
\hline $\mathrm{mpc}-\chi \cdot \mathrm{mc} \rho=0.99$ & 0.993 & 1.036 & 1.039 . & 1.041 & 1.044 \\
\hline $\operatorname{mpc} \rho=1$ & 0.596 & 0.817 & 0.862 & 0.890 & 0.962 \\
\hline $\mathrm{mpc}-\chi \cdot \mathrm{mc} \rho=1$ & 0.978 & 1.014 & 1.014 & 1.012 & 1.005 \\
\hline
\end{tabular}


Table A6. RBC: Impact responses of $m p c_{t}$ to a capital augmenting shock, the values of the hyperbola $\frac{a p c}{\sigma \sigma_{c}}$ and the sign of $d H / d z^{K},(a p c=0.718, \Psi=0)$.

\begin{tabular}{|c|c|c|c|c|c|}
\hline & $\sigma=0.3$ & $\sigma=0.9$ & $\sigma=1.4$ & $\sigma=2$ & $\sigma=10$ \\
\hline & \multicolumn{5}{|c|}{ Risk aversion $\sigma_{c}=0.5$} \\
\hline \multirow[t]{2}{*}{ Hyperbola } & 4.787 & 1.596 & 1.026 & 0.718 & 0.143 \\
\hline & $m p c(d H$-sign $)$ & $m p c(d H$-sign $)$ & $m p c(d H$-sign $)$ & $m p c(d H$-sign $)$ & $m p c(d H$-sign $)$ \\
\hline$\rho=0$ & 0.086 & 0.056 & 0.046 & 0.039 & 0.019 \\
\hline$\rho=0.95$ & 0.723 & 0.158 & -0.091 & -0.272 & -0.771 \\
\hline$\rho=0.99$ & 1.101 & 0.226 & $-0.182 \quad(+)$ & -0.474 & -1.252 \\
\hline \multirow[t]{2}{*}{$\rho=1$} & 1.268 & 0.258 & -0.225 & -0.569 & -1.467 \\
\hline & \multicolumn{5}{|c|}{ Risk aversion $\sigma_{c}=1$} \\
\hline \multirow[t]{2}{*}{ Hyperbola } & 2.394 & 0.798 & 0.513 & 0.359 & 0.072 \\
\hline & $m p c(d H-s i g n)$ & $m p c(d H$-sign $)$ & $m p c(d H$-sign $)$ & $m p c(d H$-sign $)$ & $m p c(d H$-sign $)$ \\
\hline$\rho=0$ & 0.067 & 0.040 & 0.032 & 0.027 & 0.013 \\
\hline$\rho=0.95$ & 0.702 & 0.231 & 0.049 & -0.074 & -0.375 \\
\hline$\rho=0.99$ & 1.229 & 0.402 & 0.063 & -0.155 & -0.633 \\
\hline \multirow[t]{2}{*}{$\rho=1$} & 1.515 & 0.498 & 0.070 & -0.197 & -0.754 \\
\hline & \multicolumn{5}{|c|}{ Risk aversion $\sigma_{c}=2$} \\
\hline \multirow[t]{2}{*}{ Hyperbola } & 1.197 & 0.399 & 0.257 & 0.180 & 0.036 \\
\hline & $m p c(d H-s i g n)$ & $m p c(d H$-sign $)$ & $m p c(d H$-sign $)$ & $m p c(d H$-sign $)$ & $m p c(d H$-sign $)$ \\
\hline$\rho=0$ & 0.049 & 0.027 & 0.021 & 0.018 & 0.008 \\
\hline$\rho=0.95$ & 0.653 & 0.229 & 0.091 & 0.005 & -0.179 \\
\hline$\rho=0.99$ & 1.429 & 0.477 & 0.165 & -0.006 & -0.313 \\
\hline$\rho=1$ & 2.035 & 0.622 & 0.215 & -0.013 & -0.378 \\
\hline & & & sk aversion $\sigma_{c}=$ & & \\
\hline Hyperbola & 0.598 & 0.200 & 0.128 & 0.090 & 0.018 \\
\hline & $m p c(d H$-sign $)$ & $m p c(d H$-sign $)$ & $m p c(d H$-sign $)$ & $m p c(d H$-sign $)$ & $m p c(d H$-sign $)$ \\
\hline$\rho=0$ & 0.033 & 0.017 & 0.013 & 0.011 & 0.005 \\
\hline$\rho=0.95$ & 0.570 & 0.183 & 0.082 & 0.025 & -0.085 \\
\hline$\rho=0.99$ & 1.920 & 0.473 & 0.176 & 0.042 & -0.153 \\
\hline$\rho=1$ & 4.724 & 0.798 & 0.253 & 0.053 & -0.188 \\
\hline & & & sk aversion $\sigma_{c}=$ & & \\
\hline Hyperbola & 0.299 & 0.100 & 0.064 & 0.045 & 0.009 \\
\hline & $m p c(d H$-sign $)$ & $m p c(d H$-sign $)$ & $m p c(d H$-sign $)$ & $m p c(d H$-sign $)$ & $m p c(d H$-sign $)$ \\
\hline$\rho=0$ & 0.021 & 0.010 & 0.007 & 0.006 & 0.003 \\
\hline$\rho=0.95$ & 0.454 & 0.124 & 0.057 & 0.022 & -0.041 \\
\hline$\rho=0.99$ & 5.235 & 0.408 & 0.139 & 0.042 & -0.075 \\
\hline$\rho=1$ & $-3.199^{1}$ & 0.982 & 0.223 & 0.059 & -0.093 \\
\hline
\end{tabular}

\footnotetext{
${ }^{1}$ Unlike everywhere else in this Table the negative sign implies here a positive consumption and negative income effect reflecting very strong negative impact effect on labor supply.
} 
Table A7. Generalized RBC ( Finite $\eta$ ): Impact responses of $m p c_{t}$ to a capital augmenting shock, the values of the hyperbola $\frac{a p c}{\sigma \sigma_{c}}\left(\frac{\eta-1}{\eta}\right)$ and the sign of $d H / d z^{K},(a p c=0.718, \Psi=0)$.

\begin{tabular}{|c|c|c|c|c|c|}
\hline & $\sigma=0.3$ & $\sigma=0.9$ & $\sigma=1.4$ & $\sigma=2$ & $\sigma=10$ \\
\hline & \multicolumn{5}{|c|}{ Risk aversion $\sigma_{c}=2$, price elasticity $\eta=\infty$} \\
\hline \multirow[t]{2}{*}{ Hyperbola } & 1.197 & 0.399 & 0.257 & $\mathbf{0 . 1 8 0}$ & 0.036 \\
\hline & $m p c(d H$-sign $)$ & $m p c(d H$-sign $)$ & $m p c(d H-s i g n)$ & $m p c(d H-s i g n)$ & $m p c(d H$-sign $)$ \\
\hline$\rho=0$ & $0.049 \quad(+)$ & 0.027 & $0.021 \quad(+)$ & $0.018 \quad(+)$ & 0.008 \\
\hline$\rho=0.95$ & 0.653 & 0.229 & 0.091 & $0.005 \quad(+)$ & -0.179 \\
\hline$\rho=0.99$ & 1.429 & 0.477 & 0.165 & $-0.006 \quad(+)$ & -0.313 \\
\hline \multirow[t]{2}{*}{$\rho=1$} & 2.035 & 0.622 & 0.215 & $-0.013 \quad(+)$ & -0.378 \\
\hline & \multicolumn{5}{|c|}{ Risk aversion $\sigma_{c}=2$, price elasticity $\eta=10$} \\
\hline \multirow[t]{2}{*}{ Hyperbola } & 1.077 & 0.359 & 0.231 & 0.162 & 0.032 \\
\hline & $m p c(d H-s i g n)$ & $m p c(d H-s i g n)$ & $m p c(d H$-sign $)$ & $m p c(d H-s i g n)$ & $m p c(d H$-sign $)$ \\
\hline$\rho=0$ & 0.050 & 0.028 & 0.022 & 0.018 & 0.009 \\
\hline$\rho=0.95$ & 0.663 & 0.250 & 0.116 & 0.033 & -0.144 \\
\hline$\rho=0.99$ & 1.417 & 0.518 & 0.216 & 0.048 & -0.252 \\
\hline \multirow[t]{2}{*}{$\rho=1$} & 1.983 & 0.715 & 0.281 & 0.056 & -0.305 \\
\hline & \multicolumn{5}{|c|}{ Risk aversion $\sigma_{c}=2$, price elasticity $\eta=2$} \\
\hline \multirow[t]{2}{*}{ Hyperbola } & 0.598 & 0.200 & 0.128 & 0.090 & 0.018 \\
\hline & $m p c(d H-s i g n)$ & $m p c(d H$-sign $)$ & $m p c(d H-s i g n)$ & $m p c(d H$-sign $)$ & $m p c(d H$-sign $)$ \\
\hline$\rho=0$ & 0.059 & 0.032 & 0.025 & 0.022 & 0.013 \\
\hline$\rho=0.95$ & 0.709 & 0.343 & 0.224 & 0.151 & 0.002 \\
\hline$\rho=0.99$ & 1.352 & 0.679 & 0.417 & 0.266 & -0.005 \\
\hline$\rho=1$ & 1.750 & 0.903 & 0.534 & 0.331 & -0.008 \\
\hline & & Risk aversic & $\sigma_{c}=1$, price ela & ticity $\eta=10$ & \\
\hline Hyperbola & 2.154 & 0.718 & 0.462 & 0.323 & 0.064 \\
\hline & $m p c(d H$-sign $)$ & $m p c(d H$-sign $)$ & $m p c(d H$-sign $)$ & $m p c(d H$-sign $)$ & $m p c(d H$-sign $)$ \\
\hline$\rho=0$ & 0.069 & 0.042 & 0.033 & 0.028 & 0.014 \\
\hline$\rho=0.95$ & 0.714 & 0.267 & 0.092 & -0.025 & -0.310 \\
\hline$\rho=0.99$ & 1.227 & 0.462 & 0.141 & -0.067 & -0522 \\
\hline$\rho=1$ & 1.750 & 0.571 & 0.167 & -0.089 & -0.621 \\
\hline
\end{tabular}


Table A8. Generalized RBC ( $\Psi \neq 0)$ : Impact responses of $m p c_{t}$ to a capital augmenting shock, the values of the hyperbola $\frac{a p c}{\sigma \sigma_{c}}$ and the sign of $d H / d z^{K},(a p c=0.718)$.

\begin{tabular}{|c|c|c|c|c|c|}
\hline & $\sigma=0.3$ & $\sigma=0.9$ & $\sigma=1.4$ & $\sigma=2$ & $\sigma=10$ \\
\hline Hyperbola & 1.197 & 0.399 & 0.257 & 0.180 & 0.036 \\
\hline & \multicolumn{5}{|c|}{ Risk aversion $\sigma_{c}=2$, Adjustment costs of investment $\Psi=0$} \\
\hline & $m p c(d H$-sign $)$ & $m p c(d H$-sign $)$ & $m p c(d H-s i g n)$ & $m p c(d H-s i g n)$ & $m p c(d H-$ sign $)$ \\
\hline$\rho=0$ & 0.049 & 0.027 & 0.021 & 0.018 & 0.008 \\
\hline$\rho=0.95$ & 0.653 & 0.229 & 0.091 & 0.005 & -0.179 \\
\hline$\rho=0.99$ & 1.429 & 0.477 & 0.165 & -0.006 & -0.313 \\
\hline \multirow[t]{3}{*}{$\rho=1$} & 2.035 & 0.622 & 0.215 & -0.013 & -0.378 \\
\hline & \multicolumn{5}{|c|}{ Risk aversion $\sigma_{c}=2$, Adjustment costs of investment $\Psi=0.1$} \\
\hline & $m p c(d H$-sign $)$ & $m p c(d H$-sign $)$ & $m p c(d H$-sign $)$ & $m p c(d H$-sign $)$ & $m p c(d H-$ sign $)$ \\
\hline$\rho=0$ & 0.226 & 0.208 & 0.202 & 0.198 & 0.189 \\
\hline$\rho=0.95$ & 0.700 & 0.320 & 0.187 & 0.109 & -0.097 \\
\hline$\rho=0.99$ & 1.355 & 0.548 & 0.256 & 0.085 & -0.251 \\
\hline \multirow[t]{3}{*}{$\rho=1$} & 1.828 & 0.713 & 0.303 & 0.076 & -0.327 \\
\hline & \multicolumn{5}{|c|}{ Risk aversion $\sigma_{c}=2$, Adjustment costs of investment $\Psi=1$} \\
\hline & $m p c(d H-s i g n)$ & $m p c(d H$-sign $)$ & $m p c(d H-s i g n)$ & $m p c(d H$-sign $)$ & $m p c(d H$-sign $)$ \\
\hline$\rho=0$ & $0.665 \quad(+)$ & $0.654 \quad(-)$ & $0.650 \quad(-)$ & $0.648 \quad(-)$ & $0.641 \quad(-)$ \\
\hline$\rho=0.95$ & 0.823 & 0.567 & 0.458 & 0.380 & 0.169 \\
\hline$\rho=0.99$ & 1.192 & 0.722 & 0.503 & 0.349 & -0.035 \\
\hline \multirow[t]{3}{*}{$\rho=1$} & 1.420 & 0.830 & 0.538 & 0.337 & -0.143 \\
\hline & \multicolumn{5}{|c|}{ Risk aversion $\sigma_{c}=2$, Adjustment costs of investment $\Psi=2.5$} \\
\hline & $m p c(d H$-sign $)$ & $m p c(d H$-sign $)$ & $m p c(d H-$ sign $)$ & $m p c(d H-$ sign $)$ & $m p c(d H$-sign $)$ \\
\hline$\rho=0$ & 0.816 & 0.810 & 0.808 & 0.806 & 0.802 \\
\hline$\rho=0.95$ & 0.875 & 0.684 & 0.595 & 0.529 & 0.333 \\
\hline$\rho=0.99$ & 1.133 & 0.797 & 0.622 & 0.490 & 0.110 \\
\hline \multirow[t]{3}{*}{$\rho=1$} & 1.285 & 0.877 & 0.649 & 0.476 & -0.012 \\
\hline & \multicolumn{5}{|c|}{ Risk aversion $\sigma_{c}=2$, Adjustment costs of investment $\Psi=10$} \\
\hline & $m p c(d H$-sign $)$ & $m p c(d H$-sign $)$ & $m p c(d H-s i g n)$ & $m p c(d H$-sign $)$ & $m p c(d H$-sign $)$ \\
\hline$\rho=0$ & 0.940 & 0.938 & 0.937 & 0.937 & 0.936 \\
\hline$\rho=0.95$ & 0.935 & 0.830 & 0.777 & 0.734 & 0.594 \\
\hline$\rho=0.99$ & 1.070 & 0.886 & 0.777 & 0.687 & 0.371 \\
\hline$\rho=1$ & 1.150 & 0.931 & 0.790 & 0.671 & 0.237 \\
\hline
\end{tabular}


Table A9. NK ( $\Psi=0)$ : Impact responses of $m p c_{t}$ and $m p c_{t}-v \cdot m c_{t}$ to a capital augmenting shock, values of the hyperbola $\frac{a p c}{\sigma \sigma_{c}}\left(\frac{\eta-1}{\eta}\right)$ and sign of $d H / d z^{K},\left(a p c=0.718, \sigma_{c}=2 \eta=10\right)$.

\begin{tabular}{|c|c|c|c|c|c|}
\hline & $\sigma=0.3$ & $\sigma=0.9$ & $\sigma=1.4$ & $\sigma=2$ & $\sigma=10$ \\
\hline \multirow[t]{3}{*}{ Hyperbola } & 1.077 & 0.359 & 0.231 & 0.162 & 0.032 \\
\hline & \multicolumn{5}{|c|}{ Calvo parameter $\theta=0.5$, Adjustment costs of investment $\Psi=0$} \\
\hline & $(d H$-sign $)$ & $(d H$-sign $)$ & $(d H$-sign $)$ & $m p c(d H$-sign $)$ & $($ dH-sign $)$ \\
\hline $\operatorname{mpc} \rho=0$ & 0.050 & 0.028 & 0.022 & 0.018 & 0.009 \\
\hline $\mathrm{mpc}-\mathrm{v} \cdot \mathrm{mc} \rho=0$ & 0.058 & 0.030 & 0.023 & 0.019 & 0.009 \\
\hline $\operatorname{mpc} \rho=0.95$ & 0.672 & 0.247 & 0.113 & 0.033 & -0.137 \\
\hline $\mathrm{mpc}-\mathrm{v} \cdot \mathrm{mc} \rho=0.95$ & 0.706 & 0.231 & 0.086 & -0.006 & -0.180 \\
\hline $\operatorname{mpc} \rho=0.99$ & 1.465 & 0.518 & 0.209 & 0.046 & -0.242 \\
\hline $\mathrm{mpc}-\mathrm{v} \cdot \mathrm{mc} \rho=0.99$ & 1.519 & 0.498 & 0.182 & 0.012 & -0.288 \\
\hline $\operatorname{mpc} \rho=1$ & 2.079 & 0.704 & 0.272 & 0.054 & -0.295 \\
\hline \multirow[t]{3}{*}{$\mathrm{mpc}-\mathrm{v} \cdot \mathrm{mc} \rho=1$} & 2.142 & 0.694 & 0.246 & 0.020 & -0.342 \\
\hline & \multicolumn{5}{|c|}{ Calvo parameter $\theta=0.75$, Adjustment costs of investment $\Psi=0$} \\
\hline & $(d H-s i g n)$ & $(d H-s i g n)$ & $(d H-$ sign $)$ & $m p c(d H$-sign $)$ & $(d H-s i g n)$ \\
\hline $\operatorname{mpc} \rho=0$ & 0.050 & 0.028 & 0.022 & 0.018 & 0.009 \\
\hline $\mathrm{mpc}-\mathrm{v} \cdot \mathrm{mc} \rho=0$ & 0.092 & 0.041 & 0.030 & 0.023 & 0.010 \\
\hline $\operatorname{mpc} \rho=0.95$ & 0.722 & 0.231 & 0.101 & 0.030 & -0.108 \\
\hline $\mathrm{mpc}-\mathrm{v} \cdot \mathrm{mc} \rho=0.95$ & 0.922 & 0.135 & 0.059 & -0.165 & -0.366 \\
\hline $\operatorname{mpc} \rho=0.99$ & 1.752 & 0.476 & 0.183 & 0.041 & -0.201 \\
\hline $\mathrm{mpc}-\mathrm{v} \cdot \mathrm{mc} \rho=0.99$ & 2.069 & 0.402 & $0.026 \quad(+)$ & -0.160 & -0.473 \\
\hline $\operatorname{mpc} \rho=1$ & 2.727 & 0.658 & 0.235 & 0.048 & -0.251 \\
\hline \multirow[t]{3}{*}{$\mathrm{mpc}-\mathrm{v} \cdot \mathrm{mc} \rho=1$} & 3.097 & 0.596 & 0.082 & -0.156 & -0.530 \\
\hline & \multicolumn{5}{|c|}{ Calvo parameter $\theta=0.85$, Adjustment costs of investment $\Psi=0$} \\
\hline & $(d H$-sign $)$ & $($ dH-sign $)$ & $(d H$-sign $)$ & $m p c(d H$-sign $)$ & $(d H$-sign $)$ \\
\hline $\operatorname{mpc} \rho=0$ & 0.051 & 0.028 & 0.022 & 0.018 & 0.009 \\
\hline $\mathrm{mpc}-\mathrm{v} \cdot \mathrm{mc} \rho=0$ & 0.176 & 0.067 & 0.045 & 0.034 & 0.011 \\
\hline $\operatorname{mpc} \rho=0.95$ & 0.886 & 0.199 & 0.081 & 0.026 & -0.070 \\
\hline $\mathrm{mpc}-\mathrm{v} \cdot \mathrm{mc} \rho=0.95$ & 1.487 & -0.098 & -0.412 & -0.576 & -0.870 \\
\hline $\operatorname{mpc} \rho=0.99$ & 3.426 & 0.408 & 0.141 & 0.034 & -0.141 \\
\hline $\mathrm{mpc}-\mathrm{v} \cdot \mathrm{mc} \rho=0.99$ & 4.387 & 0.181 & -0.338 & -0.581 & -0.979 \\
\hline $\operatorname{mpc} \rho=1$ & 12.304 & 0.567 & 0.179 & 0.039 & -0.183 \\
\hline $\mathrm{mpc}-\mathrm{v} \cdot \mathrm{mc} \rho=1$ & 13.427 & 0.378 & -0.288 & -0.578 & -1.039 \\
\hline
\end{tabular}


Table A10. NK: Impact responses of $m p c_{t}$ and $m p c_{t}-v \cdot m c_{t}$ to a capital augmenting shock, the values of the hyperbola $\frac{a p c}{\sigma \sigma_{c}}\left(\frac{\eta-1}{\eta}\right)$ and the sign of $d H / d z^{K},\left(a p c=0.718, \sigma_{c}=2 \eta=10\right)$.

\begin{tabular}{|c|c|c|c|c|c|}
\hline & $\sigma=0.3$ & $\sigma=0.9$ & $\sigma=1.4$ & $\sigma=2$ & $\sigma=10$ \\
\hline \multirow[t]{3}{*}{ Hyperbola } & 1.077 & 0.359 & 0.231 & 0.162 & $\mathbf{0 . 0 3 2}$ \\
\hline & \multicolumn{5}{|c|}{ Calvo parameter $\theta=0.75$, Adjustment costs of investment $\Psi=0$} \\
\hline & $(d H$-sign $)$ & $(d H$-sign $)$ & $(d H$-sign $)$ & $m p c(d H-s i g n)$ & $(d H$-sign $)$ \\
\hline $\operatorname{mpc} \rho=0$ & 0.051 & 0.028 & 0.022 & 0.018 & 0.009 \\
\hline $\mathrm{mpc}-\mathrm{v} \cdot \mathrm{mc} \rho=0$ & 0.092 & 0.041 & 0.030 & 0.023 & 0.010 \\
\hline $\operatorname{mpc} \rho=0.95$ & 0.751 & 0.229 & 0.100 & 0.030 & -0.108 \\
\hline $\mathrm{mpc}-\mathrm{v} \cdot \mathrm{mc} \rho=0.95$ & 0.949 & 0.132 & 0.060 & -0.166 & -0.366 \\
\hline $\operatorname{mpc} \rho=0.99$ & 1.945 & 0.471 & 0.181 & 0.041 & -0.201 \\
\hline $\mathrm{mpc}-\mathrm{v} \cdot \mathrm{mc} \rho=0.99$ & 2.262 & 0.397 & $0.024 \quad(+)$ & -0.160 & -0.473 \\
\hline $\operatorname{mpc} \rho=1$ & 3.239 & 0.651 & 0.233 & 0.048 & -0.251 \\
\hline \multirow[t]{3}{*}{$\mathrm{mpc}-\mathrm{v} \cdot \mathrm{mc} \rho=1$} & 3.608 & $0.589 \quad(-)$ & $0.080 \quad(+)$ & -0.154 & -0.529 \\
\hline & \multicolumn{5}{|c|}{ Calvo parameter $\theta=0.75$, Adjustment costs of investment $\Psi=0.1$} \\
\hline & (dH-sign) & $(d H$-sign $)$ & (dH-sign) & $m p c(d H$-sign $)$ & $(d H$-sign $)$ \\
\hline $\operatorname{mpc} \rho=0$ & 0.185 & 0.163 & 0.156 & 0.151 & 0.138 \\
\hline $\mathrm{mpc}-\mathrm{v} \cdot \mathrm{mc} \rho=0$ & 1.364 & 0.838 & 0.722 & 0.656 & 0.523 \\
\hline $\operatorname{mpc} \rho=0.95$ & 0.788 & 0.364 & 0.211 & 0.109 & -0.139 \\
\hline $\mathrm{mpc}-\mathrm{v} \cdot \mathrm{mc} \rho=0.95$ & 1.022 & 0.519 & 0.365 & 0.269 & 0.062 \\
\hline $\operatorname{mpc} \rho=0.99$ & 1.396 & 0.617 & 0.317 & 0.124 & -0.327 \\
\hline mpc-v $\cdot$ mc $\rho=0.99$ & 1.441 & 0.682 & 0.420 & 0.265 & -0.008 \\
\hline $\operatorname{mpc} \rho=1$ & 1.757 & 0.775 & 0.382 & 0.133 & -0.431 \\
\hline \multirow[t]{3}{*}{$\mathrm{mpc}-\mathrm{v} \cdot \mathrm{mc} \rho=1$} & 1.724 & 0.799 & 0.462 & 0.267 & -0.013 \\
\hline & Calv & parameter $\theta=0$ & 5, Adjustment $\mathrm{cc}$ & ts of investment & $=1$ \\
\hline & $(d H$-sign $)$ & $(d H-$ sign $)$ & $(d H-$ sign $)$ & $m p c(d H$-sign $)$ & $(d H$-sign $)$ \\
\hline $\operatorname{mpc} \rho=0$ & 0.570 & 0.550 & 0.543 & 0.539 & 0.526 \\
\hline $\mathrm{mpc}-\mathrm{v} \cdot \mathrm{mc} \rho=0$ & 2.221 & 1.405 & 1.246 & 0.1 .158 & 0.987 \\
\hline $\operatorname{mpc} \rho=0.95$ & 0.862 & 0.579 & 0.443 & 0.338 & 0.021 \\
\hline $\mathrm{mpc}-\mathrm{v} \cdot \mathrm{mc} \rho=0.95$ & 1.119 & 0.809 & 0.677 & 0.580 & 0.303 \\
\hline $\operatorname{mpc} \rho=0.99$ & 1.167 & 0.767 & 0.531 & 0.339 & -0.290 \\
\hline $\mathrm{mpc}-\mathrm{v} \cdot \mathrm{mc} \rho=0.99$ & 1.095 & 0.871 & 0.699 & 0.557 & 0.130 \\
\hline $\operatorname{mpc} \rho=1$ & 1.310 & 0.873 & 0.587 & 0.347 & -0.478 \\
\hline $\mathrm{mpc}-\mathrm{v} \cdot \mathrm{mc} \rho=1$ & 1.100 & 0.921 & 0.725 & 0.555 & 0.056 \\
\hline & Calv & arameter $\theta=0$ & Adjustment cos & of investment 4 & $=2.5$ \\
\hline & $(d H$-sign $)$ & $(d H-s i g n)$ & $(d H$-sign $)$ & $m p c(d H-s i g n)$ & $(d H-$ sign $)$ \\
\hline $\operatorname{mpc} \rho=0$ & 0.741 & 0.727 & 0.723 & 0.720 & 0.710 \\
\hline $\mathrm{mpc}-\mathrm{v} \cdot \mathrm{mc} \rho=0$ & 2.438 & 1.599 & 1.438 & 1.349 & 1.177 \\
\hline $\mathrm{mpc} \rho=0.95$ & 0.896 & 0.684 & 0.574 & 0.486 & 0.197 \\
\hline $\mathrm{mpc}-\mathrm{v} \cdot \mathrm{mc} \rho=0.95$ & 1.144 & 0.900 & 0.791 & 0.707 & 0.447 \\
\hline $\operatorname{mpc} \rho=0.99$ & 1.116 & 0.825 & 0.637 & 0.474 & -0.135 \\
\hline $\mathrm{mpc}-\mathrm{v} \cdot \mathrm{mc} \rho=0.99$ & 1.043 & 0.922 & 0.791 & 0.671 & 0.234 \\
\hline $\operatorname{mpc} \rho=1$ & 1.218 & 0.906 & 0.681 & 0.476 & -0.345 \\
\hline $\mathrm{mpc}-\mathrm{v} \cdot \mathrm{mc} \rho=1$ & 1.010 & 0.950 & 0.807 & 0.666 & 0.123 \\
\hline
\end{tabular}




\section{Optimal policy}
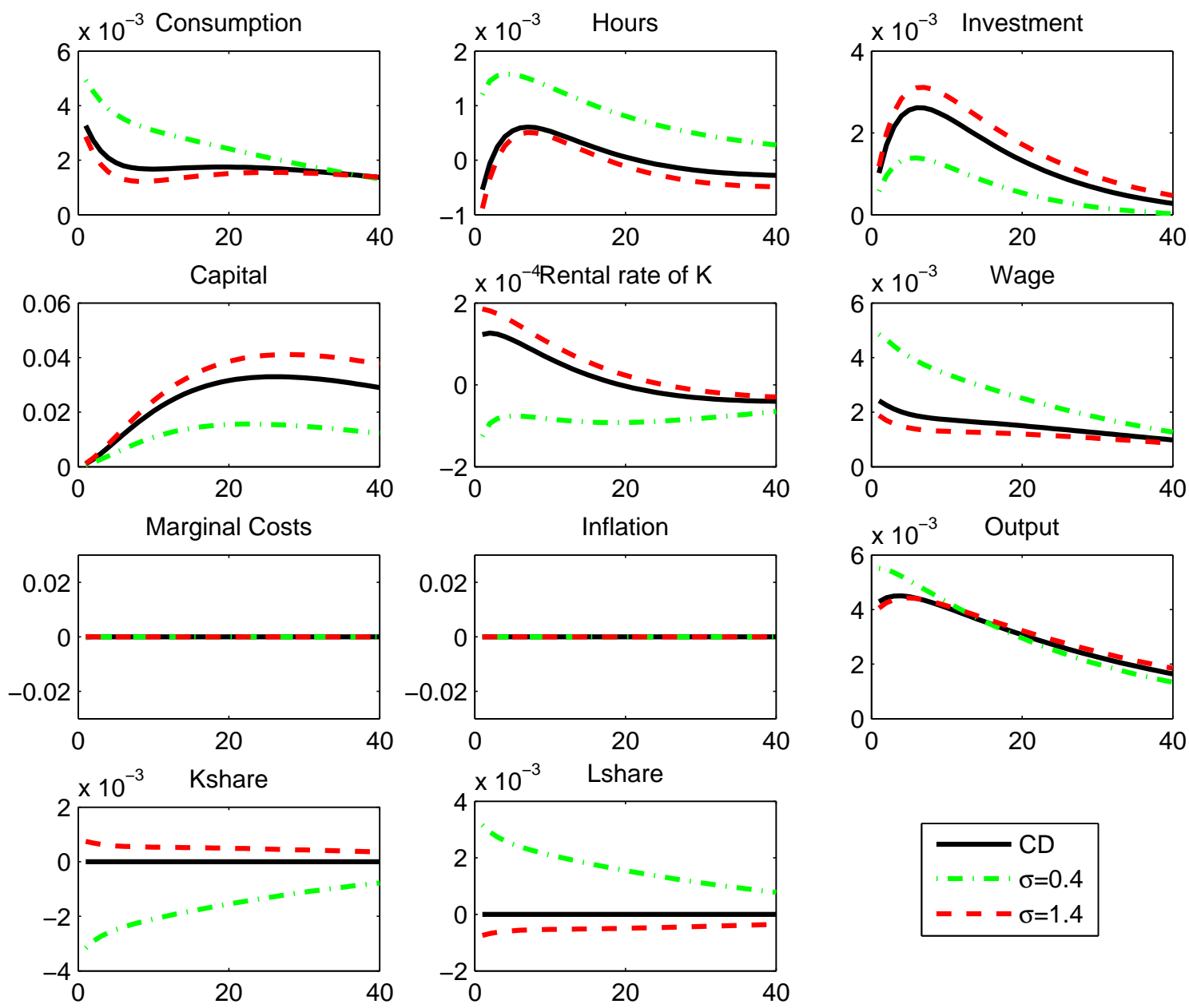

Figure 17: NK model, Ramsey Optimal Monetary Policy - Capital Augmenting Shock 



Figure 18: NK model, Ramsey Optimal Monetary Policy - Labor Augmenting Shock 

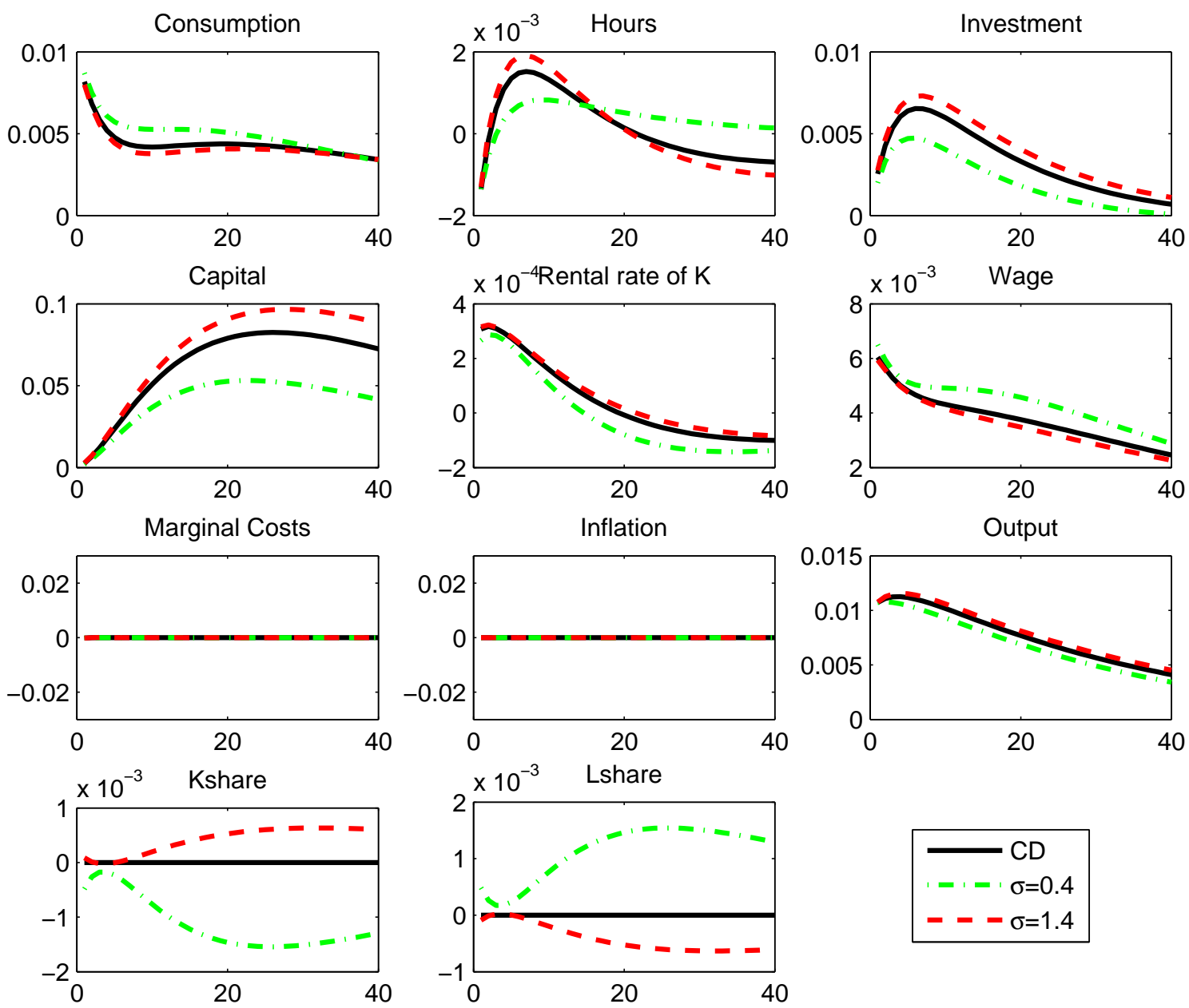

Figure 19: NK model, Ramsey Optimal Monetary Policy - Hicks Neutral Shock 


\section{E Hicks-neutral shocks}
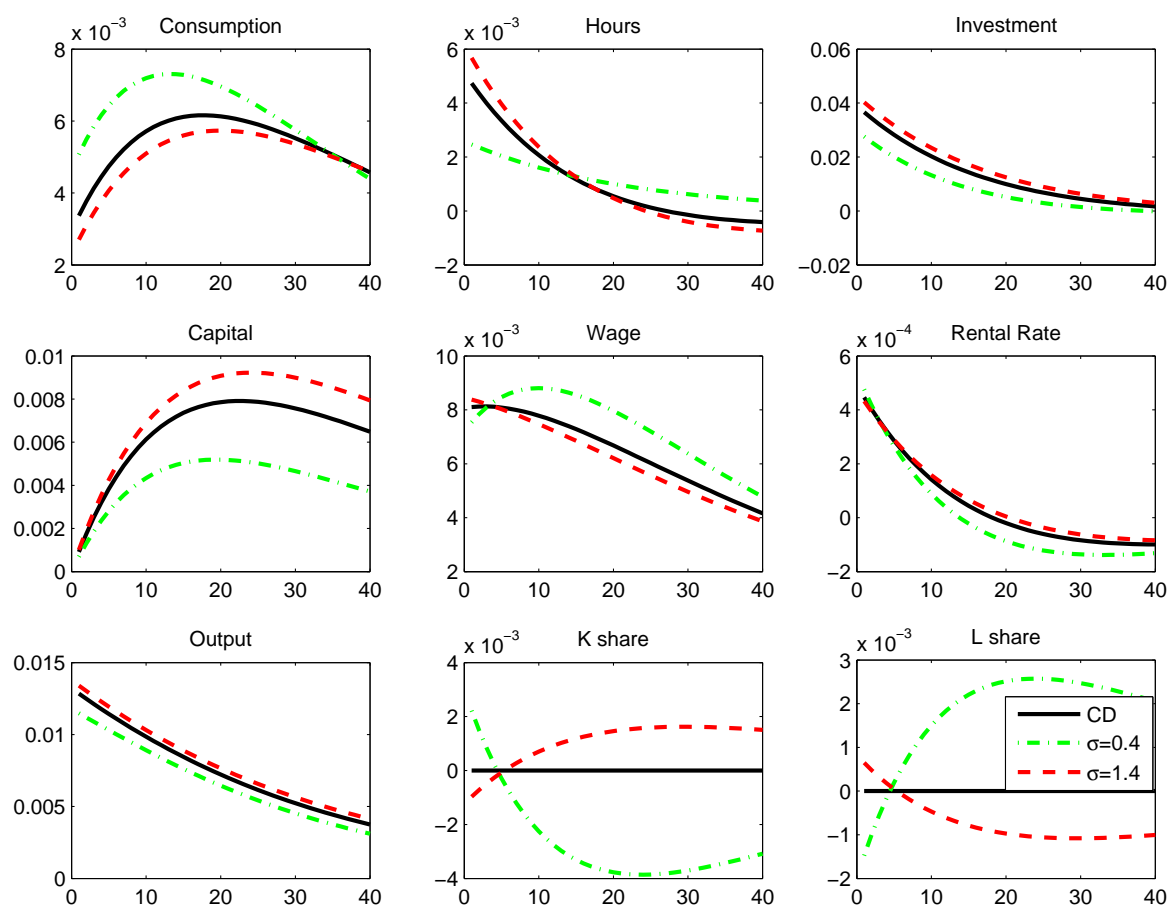

Figure 20: RBC model - Hicks Neutral Shock 

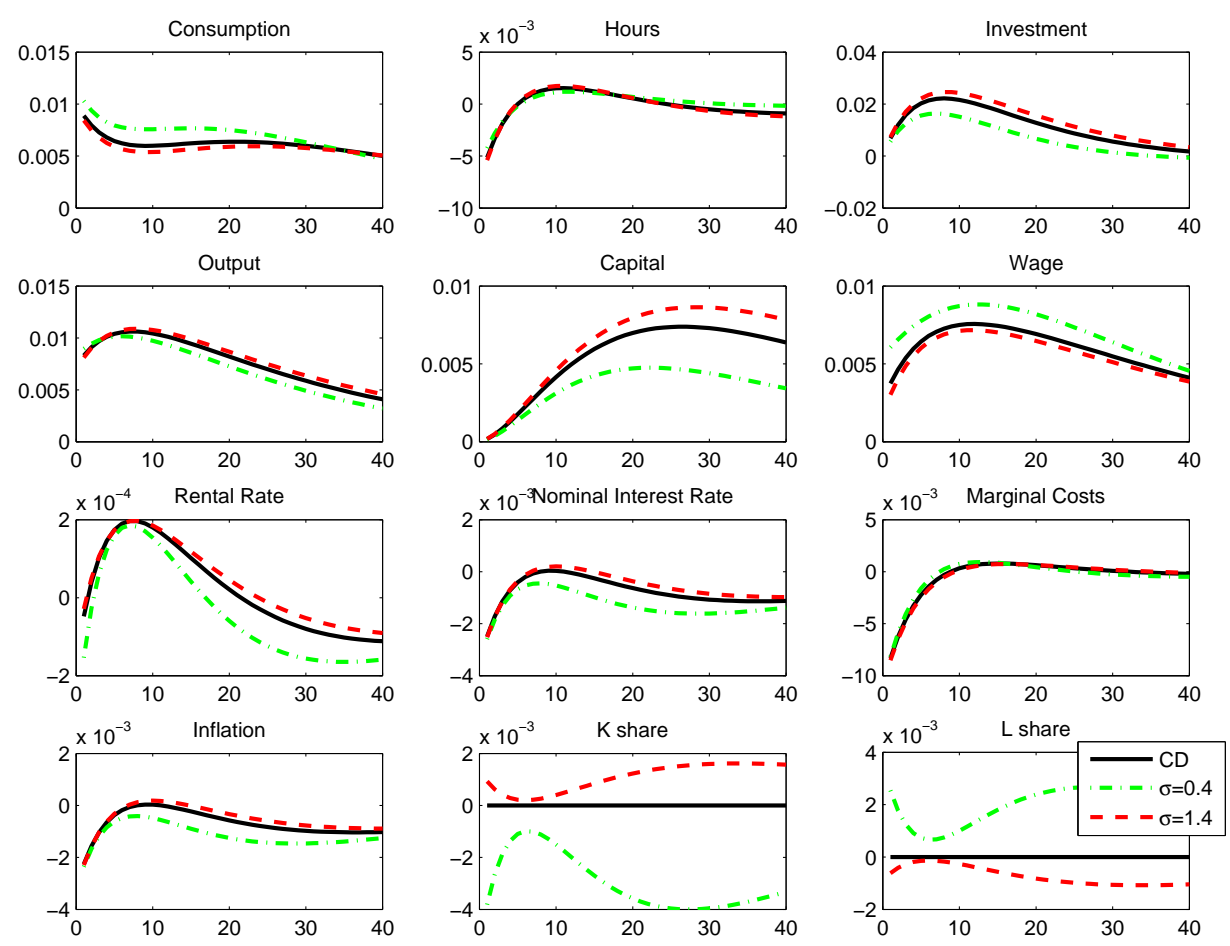

Figure 21: NK model - Hicks Neutral Shock 
